\title{
Operators with Symbol Hierarchies and Iterated Asymptotics
}

\author{
By \\ Bert-Wolfgang ScHuLzE*
}

\begin{abstract}
Ellipticity of (pseudo-differential) operators on a manifold with geometric singularities gives rise to a hierarchy of symbols, associated with the system of lowerdimensional strata of the configuration. Classical examples are boundary value problems with interior and boundary symbols (the latter ones describe Shapiro-Lopatinskij ellipticity of boundary conditions), or operators on manifolds with conical singularities with interior and conormal symbols. Ellipticity on a manifold with smooth edges may be investigated by a suitable combination of ideas from boundary value problems and cone calculus. The present article studies another typical case, namely ellipticity on a manifold that has edges with conical singularities. Locally, we may talk about cones, where the base is a manifold with smooth edges. Parametrices and iterated asymptotics of solutions to elliptic equations are determined by a three-component symbolic hierarchy, with interior, edge and conormal symbols. We construct an operator algebra of $2 \times 2$-block matrices, where the upper left corners contain the interior operators, together with so-called Green and Mellin operators (caused by analogues of Green's function in boundary value problems as well as by asymptotic phenomena), while the other entries contain trace and potential conditions with respect to the edge and pseudo-differential operators on the edge itself that are of Fuchs type with respect to the conical points. The calculus is organized in an iterative way and can be viewed as a starting point for constructing similar operator algebras with asymptotics for higher polyhedral singularities.
\end{abstract}

\section{Contents}

Introduction

Communicated by T. Kawai. Received April 18, 2001.

2000 Mathematics Subject Classification(s): 35J70, 35S05, 58J40.

Supported by the EU Research and Training Network "Geometric Analysis".

*Department of Mathematics, University of Potsdam, Box 601553, 14415 Potsdam, Germany.

e-mail: schulze@math.uni-potsdam.de 
§1. Edge Calculus with Parameters

$\S 1.1$. Cone asymptotics and Green symbols

$\S 1.2$. Mellin edge symbols

$\S 1.3$. The edge symbol algebra

$\S 1.4$. Operators on a manifold with edges

$\S 2$. Corner Symbols and Iterated Asymptotics

$\S 2.1$. Holomorphic corner symbols

$\S 2.2$. Meromorphic corner symbols and ellipticity

$\S 2.3$. Weighted corner Sobolev spaces

$\S 2.4$. Iterated asymptotics

$\S 3$. The Edge-Corner Algebra with Trace and Potential Conditions

§3.1. Green corner operators

$\S 3.2$. Smoothing Mellin corner operators

$\S 3.3$. The edge corner algebra

$\S 3.4$. Ellipticity and regularity with asymptotics

$\S 3.5$. Examples and remarks

References

\section{Introduction}

This paper is aimed at constructing a new pseudo-differential calculus on a class of manifolds $M$ with geometric singularities $M^{\prime} \subset M$, where $M^{\prime}$ consists of edges and corners (in a certain regular sense), and $M \backslash M^{\prime}$ is a $C^{\infty}$ manifold. Simpler special cases are manifolds with conical singularities or manifolds with smooth edges (the latter ones are locally defined by wedges, i.e., Cartesian products between model cones and open sets in an Euclidian space). As is known from such situations, non-smooth configurations cause an enormous variety of new structures and "unexpected" analytic and topological difficulties, cf. [26], [5], [31]. Special cases are $C^{\infty}$ manifolds $M$ with boundary $M^{\prime}=\partial M$; they can be regarded as manifolds with edges $M^{\prime}$, where $\overline{\mathbb{R}}_{+}$(the inner normal to $M^{\prime}$ with respect to some Riemannian metric) is the model cone.

Pseudo-differential algebras on (in general, pseudo-) manifolds $M$ with singularities are described by hierarchies of symbols that encode specific properties of the operators near the singularities $M^{\prime}$ (apart from their "standard" structure on the $C^{\infty}$ part $M \backslash M^{\prime}$ ), in particular, additional operators of trace and potential type on $M^{\prime}$ (for $\operatorname{dim} M^{\prime}>0$ ), cf. [29], [32]. Classical elliptic boundary value problems with or without the transmission property, mixed 
and transmission problems, and various types of crack problems belong to the realisations of the calculus with edges, cf. [28], [11]. In the past few years elliptic operators on manifolds with singularities have been intensively studied under the aspect of the index theory, cf. [6], [7] or [34], [19], and the references there. The specific nature of singularities in terms of typical differential operators needs precise definitions that distinguish regular singularities from cuspidal ones, cf. [35]. In the present paper we suppose the corner singularities to be modelled by regular cones with base manifolds that are regular manifolds with edges.

Typical differential operators may appear as Laplace-Beltrami operators for degenerate Riemannian metrics. Our corner singularities are generated by iteratively forming cones and wedges. First, let $X$ be a closed, compact $C^{\infty}$ manifold, and let $X^{\Delta}:=\left(\overline{\mathbb{R}}_{+} \times X\right) /(\{0\} \times X)$ denote the cone with base $X$. Further, let $X^{\wedge}:=\mathbb{R}_{+} \times X$ be the associated open stretched cone in a chosen splitting of variables $(r, x)$. Then, if $g_{X}(r)$ is a family of Riemannian metrics on $X$, smoothly dependent on $r$ up to $r=0$, the Laplace-Beltrami operator on $X^{\wedge}$ to the cone metric $d r^{2}+r^{2} g_{X}(r)$ has the form of a Fuchs type operator (of order $\mu=2$ ), that is

$$
A=r^{-\mu} \sum_{j=0}^{\mu} a_{j}(r)\left(-r \frac{\partial}{\partial r}\right)^{j}
$$

with operator-valued coefficients $a_{j}(r) \in C^{\infty}\left(\overline{\mathbb{R}}_{+}, \operatorname{Diff}^{\mu-j}(X)\right)$ (here, $\operatorname{Diff}^{\nu}(X)$ is the space of all differential operators on $X$ with smooth coefficients in its natural Fréchet topology; all manifolds here are supposed to be locally compact and paracompact).

Let $B$ be a manifold with conical singularities $B^{\prime}$ and $\mathbb{B}$ its stretched manifold (that is, $B^{\prime} \subset B$ is a finite subset, and $B$ is locally near a point $v \in B^{\prime}$ modelled by $X^{\Delta}$ for a closed, compact $C^{\infty}$ manifold $X$, while $\mathbb{B}$ is a $C^{\infty}$ manifold with boundary, modelled by $\overline{\mathbb{R}}_{+} \times X$ near $\partial \mathbb{B}$ ). We then have the space $\operatorname{Diff}_{\text {Fuchs }}^{\mu}(\mathbb{B})$ of all differential operators of order $\mu$ on int $\mathbb{B}$ with smooth coefficients that are of the form (0.0.1) locally near $\partial \mathbb{B}$. This definition is invariant under diffeomorphisms of $\mathbb{B}$, and $\operatorname{Diff}_{\text {Fuchs }}^{\mu}(\mathbb{B})$ is a Fréchet space in a natural way.

Next consider a Cartesian product $X^{\wedge} \times \Omega \ni(r, x, y)$ for an open set $\Omega \subseteq \mathbb{R}^{q}$ with a wedge metric $d r^{2}+r^{2} g_{X}(r, y)+d y^{2}$, where $g_{X}(r, y)$ is a family of Riemannian metrics on $X$, smoothly dependent on the variables $(r, y)$ up to $r=0$. Then the associated Laplace-Beltrami operator on $X^{\wedge} \times \Omega$ is an edge-degenerate operator (of order $\mu=2$ ). In general, an operator is said to 
be edge-degenerate, if it has the form

$$
A=r^{-\mu} \sum_{j+|\alpha| \leq \mu} a_{j \alpha}(r, y)\left(-r \frac{\partial}{\partial r}\right)^{j}\left(r D_{y}\right)^{\alpha}
$$

with coefficients $a_{j \alpha}(r, y) \in C^{\infty}\left(\overline{\mathbb{R}}_{+} \times \Omega\right.$, Diff $\left.{ }^{\mu-(j+|\alpha|)}(X)\right)$. Let $W$ be a (say, compact) manifold with edge $Y$ and $\mathbb{W}$ the stretched manifold associated with $W$ (precise definitions will be given below; roughly, $W \backslash Y$ and $Y$ are $C^{\infty}$ manifolds, and locally near every point of the edge the space $W$ is modelled by $X^{\Delta} \times \Omega$, while $\mathbb{W}$ is a $C^{\infty}$ manifold with boundary, locally near $\partial \mathbb{W}$ modelled by $\overline{\mathbb{R}}_{+} \times X \times \Omega, \Omega \subseteq \mathbb{R}^{q}$ open, where $X$ is a closed, compact $C^{\infty}$ manifold). Let $\operatorname{Diff}_{\text {edge }}^{\mu}(\mathbb{W})$ denote the space of all differential operators of order $\mu$ on int $\mathbb{W}$ with smooth coefficients that are locally near $\partial \mathbb{W}$ of the form (0.0.2). This is an invariant definition under a natural class of diffeomorphisms of $\mathbb{W}$, and $\operatorname{Diff}_{\text {edge }}^{\mu}(\mathbb{W})$ is a Fréchet space.

The spaces $M$ we are interested in are assumed to have a singular subspace $M^{\prime}$ such that $M \backslash M^{\prime}$ is $C^{\infty}$, and $M^{\prime}$ contains a finite subset $M^{\prime \prime}$, the set of corners of $M$, such that $M^{\prime} \backslash M^{\prime \prime}$ is $C^{\infty}$, too, and $V:=M \backslash M^{\prime \prime}$ is a manifold with edges $M^{\prime} \backslash M^{\prime \prime}$. Moreover, the space $M$ near any $c \in M^{\prime \prime}$ is modelled by a cone $W^{\Delta}$ for a compact manifold $W$ with edges. Such an $M$ will be called a manifold with edge-corner singularities here. More details will be given below, in particular, on the nature of transition maps belonging to different "singular charts" on $M$.

By iteratively forming cones and wedges and gluing together the local pieces we can define spaces with "higher" edge and corner singularities, generally called manifolds with singularities, though such spaces are stratified and not necessarily manifolds in the standard sense (for instance, cones with bases that are not homeomorphic to a sphere). Associated pseudo-differential algebras with symbol hierarchies as constructed in [32] are then also defined in an iterative way. Operators in those algebras are said to be elliptic, if all components of the (operator-valued) symbol tuples are pointwise bijective.

Solutions to elliptic equations are expected to have a specific asymptotic behaviour near the singularities of the configuration. Precise characteristations of such asymptotics are known in many special cases, e.g., for conical singularities, cf. [12], [22], [5], [28], edge singularities, cf. [24], [26], or corners in the sense of [27], cf. also [11], [4], [30], [14]. The problem in general is open. Asymptotics should be understood as a form of elliptic regularity, depending on an iterative system of "spectral" data of operator-valued symbols with respect to the various links of local cones and on further global data. 
The main difficulty is connected with the fact that asymptotics depend on the individual operator (analogously to "non-linear eigenvalues" $\lambda \in \mathbb{C}$ of a meromorphic function $A(\lambda)$ of operators acting in a Hilbert space; those $\lambda$ are just non-bijectivity points; for the moment, we simply talk about eigenvalues). The operator functions may depend on additional edge variables $y$; so the eigenvalues $\lambda$ may be variable and of non-constant multiplicity with respect to $y$. A calculus that is able to express asymptotics for elliptic equations in general has to integrate all individual patterns of eigenvalues. This needs an efficient approach to encode asymptotics in distribution and symbol spaces. In our paper we employ the concept of continuous asymptotics from [22], here extended to our new algebra. In particular, discrete asymptotics belong to the framework, described by meromorphic vector- and operator-valued functions. Asymptotics concern a neighbourhood of $r=0$, where $r \in \overline{\mathbb{R}}_{+}$is the axial variable of a local (stretched) cone. The invariance of our formulations refers to a fixed system of charts with transition functions of a certain specified nature. As a typical new effect we describe in this paper the interaction of edge and corner asymptotics near the corner points and characterise the nature of singular terms.

If $M$ is a manifold with corners, $V=M \backslash M^{\prime \prime}$ is a manifold with edges $M^{\prime} \backslash M^{\prime \prime}$, and we have the associated stretched manifold $\mathbb{V}$. Since $M$ is locally near a corner point $c \in M^{\prime \prime}$ of the form $([0, \varepsilon) \times W) /(\{0\} \times W)$ for some $\varepsilon>0$, we can identify $\mathbb{V}$ with $(0, \varepsilon) \times \mathbb{W}$ locally near the corresponding "stretched corner point", and we then get a stretched manifold $\mathbb{M}$ to $M$ by attaching the sets $\{0\} \times \mathbb{W}$ to $\mathbb{V}$ for every $c \in M^{\prime \prime}$, i.e., $\mathbb{M}$ is locally near a stretched corner point of the form $[0, \varepsilon) \times \mathbb{W}$; this is an invariant construction. Now we have a space $\operatorname{Diff}_{\text {corner }}^{\mu}(\mathbb{M})$ of natural differential operators on $M \backslash M^{\prime}$ defined by the property to belong to $\operatorname{Diff}_{\text {edge }}^{\mu}(\mathbb{V})$ and to have (in the splitting of variables $(t, w) \in[0, \varepsilon) \times \mathbb{W}$ near stretched corner points) the form

$$
A=t^{-\mu} \sum_{k=0}^{\mu} b_{k}(t)\left(-t \frac{\partial}{\partial t}\right)^{k}
$$

for certain operator-valued coefficients $b_{k}(t) \in C^{\infty}\left([0, \varepsilon)\right.$, Diff edge $\left.^{\mu-k}(\mathbb{W})\right)$. Operators (0.0.3) may be regarded as a "higher" version of Fuchs type operators. The elements of Diff ${ }_{\text {corner }}^{\mu}(\mathbb{M})$ will be called corner-degenerate.

If we look at the splitting of variables $w$ locally near $\partial \mathbb{W}$ into $(r, x, y) \in$ $X^{\wedge} \times \Omega$ and take a Riemannian metric on $(0, \varepsilon) \times X^{\wedge} \times \Omega \ni(t, r, x, y)$ of the form

$$
d t^{2}+t^{2}\left(d r^{2}+r^{2} g_{X}(t, r, y)+d y^{2}\right)
$$


where $g_{X}(t, r, y)$ is a family of Riemannian metrics on $X$, smoothly dependent on the variables $(t, r, y)$ up to $t=0$ and $r=0$, the associated Laplace-Beltrami operator on $(0, \varepsilon) \times X^{\wedge} \times \Omega$ belongs to Diff corner $^{\mu}\left([0, \varepsilon) \times \overline{\mathbb{R}}_{+} \times X \times \Omega\right)($ for $\mu=2)$. Corner degeneracy of an operator $A$ means in this case that we can also write

$$
A=t^{-\mu} r^{-\mu} \sum_{j+k+|\alpha| \leq \mu} a_{j k \alpha}(t, r, y)\left(-r t \frac{\partial}{\partial t}\right)^{j}\left(-r \frac{\partial}{\partial r}\right)^{k}\left(r D_{y}\right)^{\alpha}
$$

with coefficients $a_{j k \alpha}(t, r, y) \in C^{\infty}\left([0, \varepsilon) \times \overline{\mathbb{R}}_{+} \times \Omega\right.$, $\left.\operatorname{Diff}^{\mu-(j+k+|\alpha|)}(X)\right)$.

In this paper we introduce an algebra of corner-degenerate pseudo-differential operators $A$ with a principal symbol hierarchy

$$
\sigma(A)=\left(\sigma_{\psi}(A), \sigma_{\wedge}(A), \sigma_{\mathrm{c}}(A)\right)
$$

consisting of triples of interior, edge and conormal symbols, respectively. The elements of that algebra are $2 \times 2$-block matrix operators containing trace and potential operators with respect to the edges $M^{\prime} \backslash M^{\prime \prime}$. In other words, far from the corners the operators correspond to those of [29], while they are degenerate here near the corner points. The lower right entries are cone pseudo-differential operators on $M^{\prime}$ in the sense of the cone algebra of [22], [28]. We study ellipticity of operators and get parametrices within the algebra. In addition, we characterise scales of weighted spaces and subspaces with iterated edge-corner asymptotics and obtain regularity and asymptotics of solutions to elliptic operators in such spaces.

The program of this paper is as follows. In Chapter 1 we present the elements of the pseudo-differential calculus on manifolds with edges with parameters. Here, we freely use the tools from [29], see also [5]. An inspection of the material from the case without parameters shows that most of the results carry over to the parameter-dependent case (more details may be found in the author's joint paper with Maniccia [16]). For the corner theory below we need some important refinements on asymptotic data that allow us to argue in terms of Fréchet subspaces of the full edge operator algebra (with continuous asymptotics).

Chapter 2 develops the machinery of operator-valued symbols with asymptotics, referring to holomorphy or meromorphy in the complex covariable with respect to the Mellin transform in the corner axis variable (the corner is regarded as a cone, where the base is a manifold with edges). This part of the calculus, developed in Sections 2.1 and 2.2, contains simpler variants as special cases, namely, when the base is closed and $C^{\infty}$ or when it is a compact manifold with conical singularities. For the closed $C^{\infty}$ case we recover corresponding 
elements from the cone theory (see, e.g., [26], [29]); the case with conical singularities leads to the corner situation from [27]. In the present theory the closed $C^{\infty}$ case is realised in the subalgebra on $M^{\prime}$ that is a manifold with conical singularities $M^{\prime \prime}$. Another new element are the corner Sobolev spaces with asymptotics that we investigate in Sections 2.3 and 2.4. In particular, the structure of iterated edge-corner asymptotics is a feature that is respected under the pseudo-differential action with meromorphic corner symbols. It is also responsible for the nature of Green operators in the corner calculus.

Chapter 3 is devoted to the algebra of pseudo-differential operators on a manifold with edge-corner singularities. According to the general ideas of establishing operator theories on stratified spaces, cf. [32], we obtain our algebra as a conification of the edge algebra. Despite of the complexity of the edgecorner calculus, many constructions are parallel to the "usual" cone theory, see [26]. The parameter-dependent pseudo-differential calculus on a closed $C^{\infty}$ manifold is formally replaced here by the parameter-dependent edge theory. In other words, we verify that the conification concept really works in the case of a cone when the base is a manifold with edges.

Observe that manifolds $M$ with conical points and boundary are particular manifolds with edge-corner singularities in the sense of this paper. The edge is then the boundary $M^{\prime}=\partial M$ with its conical singularities $M^{\prime \prime}$ (that are just the corner points) and corner bases that are simply compact $C^{\infty}$ manifolds with boundary. In this case our theory is a calculus of boundary value problems, see Kondrat'ev [12] for the case of differential operators. The interior symbols are edge-degenerate along the smooth part $M^{\prime} \backslash M^{\prime \prime}$ of the boundary. Special such symbols (modulo smoothing ones) are symbols that are $C^{\infty}$ up to $M^{\prime} \backslash M^{\prime \prime}$. Another (narrower, though interesting) class of interior symbols are those with the transmission property at $M^{\prime} \backslash M^{\prime \prime}$ which is just the assumption in [12] as well as in the pseudo-differential algebra of boundary value problems in the author's joint paper with Schrohe [20], [21], based on the calculus of Boutet de Monvel [3] (see also Rempel and Schulze [17]) and the cone algebra of Rempel and Schulze [18] (see also [25], [26]).

Another special case of our theory are Sobolev type problems. Classical Sobolev problems are posed for elliptic equations in a domain like $G \backslash C$, where $G$ is an open set in $\mathbb{R}^{n}$ and $C$ a closed $C^{\infty}$ submanifold of codimension $>1$. On $C$ there are posed elliptic trace (and potential) conditions that can be viewed as edge conditions. Problems of a similar type have been originally studied by Sobolev [36] and later on in a certain operator algebra framework by Sternin [37]. The case when $C$ is a manifold with singularities is interesting as well. 
In particular, if $C$ has conical singularities, our calculus is a framework to get parametrices and the Fredholm property to elliptic Sobolev problems.

Edge-corner singularities are very natural in various models of physics. For instance, long-time asymptotics of solutions of boundary value problems to parabolic equations in domains with edges can be expressed by our methods where a variant for boundary value problems is to be employed, together with an interpretation of the time as an (anisotropic) corner axis variable, see also the author's joint papers with Krainer [14], [13] concerning results in this direction under other geometric assumptions.

As noted before our calculus as a cone theory for a base with edges employs the parameter-dependent edge algebra. Tools of that theory may also be found in Behm [2] and Dorschfeldt [4]. Parameters (in anisotropic form) may occur as spectral variables in elliptic differential operators. If the operators are given on a manifold with conical singularities, spectral parameters play the role of edge covariables. This has been applied by Gil [8] for studying heat trace expansions, using results of [27]. A similar program makes sense for operators on manifolds with edges with the spectral variable as parameter.

Let us finally note that when $B_{i}$ are manifolds with conical singularities $S_{i}$, $i=1,2$, the Cartesian product $M:=B_{1} \times B_{2}$ is a manifold with edge-corner singularities $S_{1} \times S_{2}$. It would be interesting to investigate external products of elliptic operators (or of complexes) on $B_{1}$ and $B_{2}$ (say, for compact $B_{1}, B_{2}$ ) and to establish a Künneth formula for the index in terms of the theories of elliptic operators on $B_{1}, B_{2}$ and $B_{1} \times B_{2}$.

\section{$\S 1$ Edge Calculus with Parameters}

\section{$\S 1.1$. Cone asymptotics and Green symbols}

A manifold $W$ with edges $Y \subset W$ is defined by the following data:

(i) $W \backslash Y$ and $Y$ are $C^{\infty}$ manifolds.

(ii) Every $y \in Y$ has a neighbourhood $V$ in $W$ that is homeomorphic to a wedge $X^{\Delta} \times \Omega$ for a closed compact $C^{\infty}$ manifold $X$ and an open set $\Omega \subseteq \mathbb{R}^{q}, q=\operatorname{dim} Y$; any such homeomorphism $\varphi: V \rightarrow X^{\Delta} \times \Omega$ is said to be a singular chart (near the edge).

(iii) Each singular chart $\varphi: V \rightarrow X^{\Delta} \times \Omega$ induces diffeomorphisms $\varphi_{0}:=$ $\left.\varphi\right|_{V \backslash Y}: V \backslash Y \rightarrow X^{\wedge} \times \Omega$ and $\varphi^{\prime}:=\left.\varphi\right|_{V \cap Y}: V \cap Y \rightarrow \Omega$; furthermore, if $\widetilde{\varphi}: \widetilde{V} \rightarrow X^{\Delta} \times \widetilde{\Omega}$ is another singular chart with $V \cap \widetilde{V} \neq \emptyset$ the transition map $\widetilde{\varphi}_{0} \varphi_{0}^{-1}: X^{\wedge} \times \Sigma \rightarrow X^{\wedge} \times \widetilde{\Sigma}$ is the restriction of a diffeomorphism 
$\mathbb{R} \times X \times \Sigma \rightarrow \mathbb{R} \times X \times \widetilde{\Sigma}$ to $X^{\wedge} \times \Sigma$; here, $\Sigma:=\varphi^{\prime}(V \cap \widetilde{V} \cap Y), \widetilde{\Sigma}:=$ $\widetilde{\varphi}^{\prime}(V \cap \widetilde{V} \cap Y)$.

$X^{\wedge}=\mathbb{R}_{+} \times X$ is called the (open stretched) model cone of the corresponding (open stretched) wedge $X^{\wedge} \times \Omega$ and $X$ its base.

For simplicity, throughout this exposition we assume $X$ to be the same for all $y \in Y$ and the transition maps to only depend on $y$ in a neighbourhood of $r=0$; here, $(r, x, y)$ denotes the local splitting of variables in $X^{\wedge} \times \Omega$.

A manifold $W$ with edges $Y$ gives rise to an associated stretched manifold $\mathbb{W}$ that is a $C^{\infty}$ manifold with boundary $\partial \mathbb{W}$, where $\mathbb{W} \backslash \partial \mathbb{W}$ is diffeomorphic to $W \backslash Y$, and $\mathbb{W}$ is generated from $W \backslash Y$ by attaching the sets $\{0\} \times X \times \Omega$ to $V \backslash Y$, cf. (ii); this is an invariant construction. Then $\partial \mathbb{W}$ is an $X$-bundle on $Y$. For references below we fix a collar neighbourhood $\cong[0,1) \times \partial \mathbb{W}$ of $\partial \mathbb{W}$ in $\mathbb{W}$ with the normal variable $r \in[0,1)$ and a function $\mathrm{h} \in C^{\infty}(\mathbb{W} \backslash \partial \mathbb{W})$ that is strictly positive, where $\mathrm{h}=r$ for $0<r<\varepsilon$ for some $0<\varepsilon<1$.

We now formulate operator-valued symbols on an open set $U \subseteq \mathbb{R}^{p}$ with values in a space of so-called Green operators on $X^{\wedge}$ that encode a part of the asymptotic information of our wedge operator calculus.

The symbols refer to weighed Sobolev spaces $\mathcal{K}^{s, \gamma}\left(X^{\wedge}\right), s, \gamma \in \mathbb{R}$, that are defined as follows. First consider the Mellin transform $(M u)(z)=\int_{0}^{\infty} r^{z-1} u(r) d r$ acting on (vector-valued) distributions $u(r)$ on $\mathbb{R}_{+}$belonging to specific spaces that become clear in the context. In particular, we may take $u \in C_{0}^{\infty}\left(\mathbb{R}_{+}, C^{\infty}(X)\right)$. Let $L_{\mathrm{cl}}^{\mu}\left(X ; \mathbb{R}^{l}\right)$ denote the space of all classical parameter-dependent pseudodifferential operators on $X$ of order $\mu$, that is, the local symbols are classical with respect to the covariables $(\xi, \lambda) \in \mathbb{R}^{n+l}, n=\operatorname{dim} X$, and $L^{-\infty}\left(X ; \mathbb{R}^{l}\right):=$ $\bigcap_{\mu} L_{\mathrm{cl}}^{\mu}\left(X ; \mathbb{R}^{l}\right)=\mathcal{S}\left(\mathbb{R}^{l}, L^{-\infty}(X)\right)$, where $L^{-\infty}(X)$ is identified with $C^{\infty}(X \times X)$ via a chosen Riemannian metric on $X$. We employ the known fact that for every $\mu \in \mathbb{R}$ there exists an element $R^{\mu}(\lambda) \in L_{\mathrm{cl}}^{\mu}\left(X ; \mathbb{R}^{l}\right)$ that induces isomorphisms

$$
R^{\mu}(\lambda): H^{s}(X) \rightarrow H^{s-\mu}(X)
$$

between the standard Sobolev spaces on $X$ of smoothness $s$, for all $s \in \mathbb{R}$ and $\lambda \in \mathbb{R}^{l}$.

Set $\Gamma_{\beta}:=\{z \in \mathbb{C}: \operatorname{Re} z=\beta\}$ for $\beta \in \mathbb{R}$. Then $\mathcal{H}^{s, \gamma}\left(X^{\wedge}\right)$ denotes the completion of $C_{0}^{\infty}\left(\mathbb{R}_{+}, C^{\infty}(X)\right)$ with respect to the norm

$$
\left\{\frac{1}{2 \pi i} \int_{\Gamma_{\frac{n+1}{2}-\gamma}}\left\|R^{s}(\operatorname{Im} z)(M u)(z)\right\|_{L^{2}(X)}^{2} d z\right\}^{\frac{1}{2}}
$$


where $R^{s}(\varrho) \in L_{\mathrm{cl}}^{\mu}\left(X ; \mathbb{R}_{\varrho}\right)$ is an element that induces isomorphisms in the Sobolev spaces on $X$ (different choices of such order reducing families give rise to equivalent norms). We now define

$$
\mathcal{K}^{s, \gamma}\left(X^{\wedge}\right)=\left\{\omega u+(1-\omega) v: u \in \mathcal{H}^{s, \gamma}\left(X^{\wedge}\right), v \in H_{\text {cone }}^{s}\left(X^{\wedge}\right)\right\} .
$$

Here, $\omega(r)$ is a cut-off function (that is, $\omega \in C_{0}^{\infty}\left(\overline{\mathbb{R}}_{+}\right)$and $\omega(r)=1$ in a neighbourhood of $r=0)$, and $H_{\text {cone }}^{s}\left(X^{\wedge}\right)$ is the subspace of all $v \in H_{\text {loc }}^{s}(\mathbb{R} \times$ $X)\left.\right|_{X^{\wedge}}$ such that $\left(\chi^{*}\right)^{-1} \varphi(x)(1-\omega(r)) v \in H^{s}\left(\mathbb{R}^{n+1}\right)$ for every $\varphi \in C^{\infty}(X)$ supported in a coordinate neighbourhood $U$ on $X$, for any diffeomorphism $\chi: \mathbb{R}_{+} \times U \rightarrow \mathbb{R}_{+} \times \Sigma$ of the form $\chi(r, x)=\left(r, \chi_{1}(x)\right)$ for a diffeomorphism $\chi_{1}: U \rightarrow \Sigma$ to an open set $\Sigma$ on the unit sphere $S^{n}$ in $\mathbb{R}^{n+1}$.

If a Fréchet space $E$ is a (left) module over an algebra $A$, the completion of $\{a e: e \in E\}$ in $E$ for an $a \in A$ is denoted by $[a] E$; analogously, we can form $E[a]$ or $[a] E[b]$ when $E$ is a right or two-sided module over $A$. Moreover, if $E_{0}$ and $E_{1}$ are Fréchet spaces embedded in a Hausdorff topological vector space $H$, we endow $E_{0}+E_{1}=\left\{e_{0}+e_{1}: e_{0} \in E_{0}, e_{1} \in E_{1}\right\}$ with the Fréchet topology from the bijection $E_{0}+E_{1} \cong E_{0} \oplus E_{1} / \Delta, \Delta:=\left\{(e,-e): e \in E_{0} \cap E_{1}\right\}$, where the space on the right is taken in the quotient topology. We then call $E_{0}+E_{1}$ the non-direct sum of Fréchet spaces. In particular, if $E_{0}$ and $E_{1}$ are Hilbert spaces, also $E_{0}+E_{1}$ is a Hilbert space under the identification with the orthogonal complement of $\Delta$ in $E_{0} \oplus E_{1}$.

In this sense we can write $\mathcal{K}^{s, \gamma}\left(X^{\wedge}\right)=[\omega] \mathcal{H}^{s, \gamma}\left(X^{\wedge}\right)+[1-\omega] H_{\text {cone }}^{s}\left(X^{\wedge}\right)$.

The spaces $\mathcal{H}^{s, \gamma}\left(X^{\wedge}\right)$ and $\mathcal{K}^{s, \gamma}\left(X^{\wedge}\right)$ are systematically employed in [26], see also [29]. We have

$$
\mathcal{K}^{0,0}\left(X^{\wedge}\right)=\mathcal{H}^{0,0}\left(X^{\wedge}\right)=r^{-\frac{n}{2}} L^{2}\left(\mathbb{R}_{+} \times X\right)
$$

with $L^{2}\left(\mathbb{R}_{+} \times X\right)$ being taken with the measure $d r d x, d x$ associated with a Riemannian metric on $X$. We will mainly consider the spaces $\mathcal{K}^{s, \gamma}\left(X^{\wedge}\right)$ on the infinite cone. Note that $\omega r^{\delta} \mathcal{K}^{s, \gamma}\left(X^{\wedge}\right)=\omega \mathcal{K}^{s, \gamma+\delta}\left(X^{\wedge}\right)$ for every $s, \gamma, \delta \in \mathbb{R}$. Moreover, setting $\kappa_{\lambda}: u(r, x) \rightarrow \lambda^{(n+1) / 2} u(\lambda r, x), \lambda \in \mathbb{R}_{+}$, we have a strongly (in $\lambda \in \mathbb{R}_{+}$) continuous group $\left\{\kappa_{\lambda}\right\}_{\lambda \in \mathbb{R}_{+}}$of isomorphisms

$$
\kappa_{\lambda}: \mathcal{K}^{s, \gamma}\left(X^{\wedge}\right) \rightarrow \mathcal{K}^{s, \gamma}\left(X^{\wedge}\right)
$$

for every $s, \gamma \in \mathbb{R}$.

If $E$ is a Hilbert space and $\left\{\kappa_{\lambda}\right\}_{\lambda \in \mathbb{R}_{+}}$a strongly continuous group of isomorphisms, $\kappa_{\lambda}: E \rightarrow E, \lambda \in \mathbb{R}_{+}$(such that $\kappa_{\lambda} \kappa_{\lambda^{\prime}}=\kappa_{\lambda \lambda^{\prime}}$ for all $\lambda, \lambda^{\prime} \in \mathbb{R}_{+}$ and $\kappa_{1}=\mathrm{id}_{E}$ ), we say that $E$ is endowed with a group action. More generally, if a Fréchet space $E$ is written as a projective limit of Hilbert spaces $\varliminf_{j \in \mathbb{N}} E^{j}$, 
with continuous embeddings $E^{j+1} \hookrightarrow E^{j}$ for all $j$, and if $\left\{\kappa_{\lambda}\right\}_{\lambda \in \mathbb{R}_{+}}$is a group action on $E^{0}$ that restricts to group actions on $E^{j}$ for all $j$, we say that $E$ is endowed with a group action. We also admit the case $E=\mathbb{C}^{N} ;$ then the group actions are always supposed to be of the form $\kappa_{\lambda} e:=\lambda^{\alpha} e, e \in E$, for a certain $\alpha \in \mathbb{R} \backslash\{0\}$ (the value of $\alpha$ is given by the context). If group actions $\left\{\kappa_{\lambda}\right\}_{\lambda \in \mathbb{R}_{+}}$ and $\left\{\varphi_{\lambda}\right\}_{\lambda \in \mathbb{R}_{+}}$are given on spaces $E$ and $F$, respectively, on $E \oplus F$ we choose the group action $\operatorname{diag}\left(\kappa_{\lambda}, \varphi_{\lambda}\right)$, defined by $u \oplus v \rightarrow \kappa_{\lambda} u \oplus \varphi_{\lambda} v$ for all $u \in E$, $v \in F$.

Let $\left(E,\left\{\kappa_{\lambda}\right\}_{\lambda \in \mathbb{R}_{+}}\right)$and $\left(\widetilde{E},\left\{\widetilde{\kappa}_{\lambda}\right\}_{\lambda \in \mathbb{R}_{+}}\right)$be Hilbert spaces with fixed group actions. Then

$$
S^{\mu}\left(U \times \mathbb{R}^{q} ; E, \widetilde{E}\right)
$$

for $U \subset \mathbb{R}^{p}$ open, $\mu \in \mathbb{R}$, denotes the subspace of all $a(y, \eta) \in C^{\infty}(U \times$ $\left.\mathbb{R}^{q}, \mathcal{L}(E, \widetilde{E})\right)$ such that

$$
\left\|\widetilde{\kappa}_{\langle\eta\rangle}^{-1}\left\{D_{y}^{\alpha} D_{\eta}^{\beta} a(y, \eta)\right\} \kappa_{\langle\eta\rangle}\right\|_{\mathcal{L}(E, \widetilde{E})} \leq c\langle\eta\rangle^{\mu-|\beta|}
$$

for every $\alpha \in \mathbb{N}^{p}, \beta \in \mathbb{N}^{q}, y \in K$, for arbitrary $K \subset \subset U, \eta \in \mathbb{R}^{q}$, with constants $c=c(\alpha, \beta, K)>0$. The best possible constants form a semi-norm system in the space (1.1.2) which is then a Fréchet space.

If $S^{(\mu)}\left(U \times\left(\mathbb{R}^{q} \backslash\{0\}\right) ; E, \widetilde{E}\right)$ denotes the set of all $f(y, \eta) \in C^{\infty}\left(U \times\left(\mathbb{R}^{q} \backslash\right.\right.$ $\{0\}) ; E, \widetilde{E})$ such that $f(y, \lambda \eta)=\lambda^{\mu} \widetilde{\kappa}_{\lambda} f(y, \eta) \kappa_{\lambda}^{-1}$ for all $\lambda \in \mathbb{R}_{+}$and all $y \in U$, $\eta \neq 0$, we have

$$
\chi S^{(\mu)}\left(U \times\left(\mathbb{R}^{q} \backslash\{0\}\right) ; E, \widetilde{E}\right) \subset S^{\mu}\left(U \times \mathbb{R}^{q} ; E, \widetilde{E}\right)
$$

for every excision function $\chi(\eta) \in C^{\infty}\left(\mathbb{R}^{q}\right)$ (that is, $\chi(\eta)=0$ in a neighbourhood of $\eta=0, \chi(\eta)=1$ for $|\eta|>c$ for a constant $c>0)$.

Let $S_{\mathrm{cl}}^{\mu}\left(U \times \mathbb{R}^{q} ; E, \widetilde{E}\right)$ denote the subspace of all $a(y, \eta) \in S^{\mu}\left(U \times \mathbb{R}^{q} ; E, \widetilde{E}\right)$ such that there are elements $a_{(\mu-j)}(y, \eta) \in S^{(\mu-j)}\left(U \times\left(\mathbb{R}^{q} \backslash\{0\}\right) ; E, \widetilde{E}\right), j \in \mathbb{N}$, satisfying.

$$
r_{N}(y, \eta):=a(y, \eta)-\chi(\eta) \sum_{j=0}^{N} a_{(\mu-j)}(y, \eta) \in S^{\mu-(N+1)}\left(U \times \mathbb{R}^{q} ; E, \widetilde{E}\right)
$$

for all $N \in \mathbb{N}$, where $\chi$ is any excision function. The semi-norms of the (uniquely determined) $a_{(\mu-j)}(y, \eta), j \in \mathbb{N}$, together with the semi-norms of remainders $r_{N}(y, \eta)$ in (1.1.3) can be taken as a semi-norm system in $S_{\mathrm{cl}}^{\mu}(U \times$ $\left.\mathbb{R}^{q} ; E, \widetilde{E}\right)$ such that this space is Fréchet.

If a definition or relation makes sense both for non-classical or classical objects, we write "(cl)" as subscript. If $E$ or $\widetilde{E}$ are Fréchet spaces with group 
actions, we can also define $S_{(\mathrm{cl})}^{\mu}\left(U \times \mathbb{R}^{q} ; E, \widetilde{E}\right)$ in a reasonable way, cf. [32]. In particular, if $E$ is a Hilbert, $\widetilde{E}=\varliminf_{j \in \mathbb{N}} \widetilde{E}^{j}$ a Fréchet space, we have continuous embeddings $S_{(\mathrm{cl})}^{\mu}\left(U \times \mathbb{R}^{q} ; E, \widetilde{E}^{j+1}\right) \hookrightarrow S_{(\mathrm{cl})}^{\mu}\left(U \times \mathbb{R}^{q} ; E, \widetilde{E}^{j}\right)$ for all $j$, and we then set

$$
S_{(\mathrm{cl})}^{\mu}\left(U \times \mathbb{R}^{q} ; E, \widetilde{E}\right):=\lim _{\longleftarrow} S_{(\mathrm{cl})}^{\mu}\left(U \times \mathbb{R}^{q} ; E, \widetilde{E}^{j}\right) .
$$

The pseudo-differential calculus on a wedge $X^{\wedge} \times \Omega \ni(r, x, y)$ is a calculus with operator-valued symbols that refer to spaces

$$
E=\mathcal{K}^{s, \gamma}\left(X^{\wedge}\right) \oplus \mathbb{C}^{j_{-}}, \quad \widetilde{E}=\mathcal{K}^{s-\mu, \gamma-\mu}\left(X^{\wedge}\right) \oplus \mathbb{C}^{j_{+}}
$$

for all $s \in \mathbb{R}$, and fixed $\gamma \in \mathbb{R}$, with the group actions

$$
\operatorname{diag}\left(\kappa_{\lambda}, \varphi_{\lambda}\right)
$$

with the above-mentioned $\kappa_{\lambda}$ and $\varphi_{\lambda} e:=\lambda^{(n+1) / 2} e$ for $e \in \mathbb{C}^{j_{ \pm}}, \lambda \in \mathbb{R}_{+}$(recall that $n=\operatorname{dim} X)$. In the present section we replace $\widetilde{E}$ by suitable Fréchet subspaces with (discrete or continuous) asymptotics in the first component. The subspaces $\mathcal{K}_{P}^{s, \gamma}\left(X^{\wedge}\right)$ of $\mathcal{K}^{s, \gamma}\left(X^{\wedge}\right)$ depend on chosen asymptotic types $P$, associated with weight data $(\gamma, \Theta)$ and a "weight interval" $\Theta=(\vartheta, 0]$ for some $-\infty \leq \vartheta<0$. We first have the space of functions of "flatness $\Theta$ " relative to the weight $\gamma$

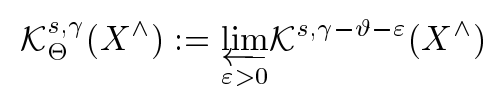

(clearly, $\mathcal{K}_{\Theta}^{s, \gamma}\left(X^{\wedge}\right)=\mathcal{K}^{s, \infty}\left(X^{\wedge}\right)$ for infinite $\left.\Theta\right)$. Given any $B \subseteq \mathbb{R}$ we set $S_{B}=\{z \in \mathbb{C}: \operatorname{Re} z \in B\}$. Let $\operatorname{As}\left(X, \boldsymbol{g}^{\bullet}\right)$ for $\boldsymbol{g}=(\gamma, \Theta)$ denote the set of all so-called discrete asymptotic types

$$
P=\left\{\left(p_{j}, m_{j}, L_{j}\right)\right\}_{0 \leq j \leq N},
$$

where $N=N(P) \leq \infty$ and $N(P)<\infty$ for finite $\Theta$, defined by the following properties: $\pi_{\mathbb{C}} P:=\left\{p_{j}\right\}_{0 \leq j \leq N} \subset S_{((n+1) / 2-\gamma+\vartheta,(n+1) / 2-\gamma)}$ for $n=\operatorname{dim} X$, $\operatorname{Re} p_{j} \rightarrow-\infty$ as $j \rightarrow \infty$ for $N(P)=\infty, m_{j} \in \mathbb{N}$, and $L_{j} \subset C^{\infty}(X)$ a finitedimensional subspace. We also admit the trivial asymptotic type $\Theta$ as an element of $\operatorname{As}\left(X, \boldsymbol{g}^{\bullet}\right)$ characterised by $\pi_{\mathbb{C}} \Theta=\emptyset$.

We then define $\mathcal{K}_{P}^{s, \gamma}\left(X^{\wedge}\right)$ to be the subspace of all $u(r, x) \in \mathcal{K}^{s, \gamma}\left(X^{\wedge}\right)$ such that there are coefficients $c_{j k}(x) \in L_{j}, 0 \leq k \leq m_{j}, 0 \leq j \leq N$, such that in the case $\Theta=(-\infty, 0]$ for every $0 \leq \beta \leq-\vartheta$ there is an $M(\beta)$ such that

$$
u(r, x)-\omega(r) \sum_{j=0}^{M} \sum_{k=0}^{m_{j}} c_{j k}(x) r^{-p_{j}} \log ^{k} r \in \mathcal{K}^{s, \gamma+\beta}\left(X^{\wedge}\right)
$$


for all $M \geq M(\beta)$, where $\omega$ is an arbitrary cut-off function. For finite $\Theta$ we similarly require $u(r, x)-\sum_{j=0}^{N} \sum_{k=0}^{m_{j}} c_{j k}(x) r^{-p_{j}} \log ^{k} r \in \mathcal{K}_{\Theta}^{s, \gamma}\left(X^{\wedge}\right)$ for $N=$ $N(P)$.

Next we describe what we understand by continuous asymptotics, first for a finite weight interval $\Theta$.

If $U \subseteq \mathbb{C}$ is an open set and $E$ a Fréchet space, $\mathcal{A}(U, E)$ denotes the space of all $E$-valued holomorphic functions in $U$. Further, if $K \subset \mathbb{C}$ is any compact set, $\mathcal{A}^{\prime}(K, E)$ denotes the space of all $E$-valued analytic functionals carried by $K$. Both $\mathcal{A}(U, E)$ and $\mathcal{A}^{\prime}(K, E)$ are considered with their corresponding natural (nuclear) Fréchet topologies. Let $\mathcal{V}$ defined to be the system of all closed subsets $V \subset \mathbb{C}$ such that $V \cap\left\{z: c \leq \operatorname{Re} z \leq c^{\prime}\right\}$ is compact for every $c \leq c^{\prime}$, and $V=V^{I}:=\left\{z=(1-\lambda) z_{0}+\lambda z_{1}: z_{0}, z_{1} \in V\right.$ with $\left.\operatorname{Re} z_{0}=\operatorname{Re} z_{1}, 0 \leq \lambda \leq 1\right\}$. Given a compact set $K \in \mathcal{V}$ contained in $\{z: \operatorname{Re} z \leq(n+1) / 2-\gamma\}$ we form the space $\mathcal{A}^{\prime}\left(K, C^{\infty}(X)\right)$ and set

$$
\mathcal{E}_{K}\left(X^{\wedge}\right):=\left\{\omega(r)\left\langle\zeta_{w}, r^{-w}\right\rangle: \zeta \in \mathcal{A}^{\prime}\left(K, C^{\infty}(X)\right\}\right.
$$

for a fixed cut-off function $\omega(r)$. The space (1.1.7) is contained in $\mathcal{K}^{\infty, \gamma}\left(X^{\wedge}\right)$.

Let $M_{\delta}$ denote the weighted Mellin transform, defined by $\left(M_{\delta} u\right)(z):=$ $M\left(r^{-\delta} u\right)(z+\delta)$. Then, for $\delta<\gamma-n / 2$ we get a subspace $M_{\delta}\left(\mathcal{E}_{K}\left(X^{\wedge}\right)\right) \subset \mathcal{A}(\mathbb{C} \backslash$ $\left.K, C^{\infty}(X)\right)$ that is isomorphic to $\mathcal{A}^{\prime}\left(K, C^{\infty}(X)\right)$; thus there is an isomorphism $\mathcal{E}_{K}\left(X^{\wedge}\right) \cong \mathcal{A}^{\prime}\left(K, C^{\infty}(X)\right)$. Write $u \sim v$ for $u, v \in \mathcal{E}_{K}\left(X^{\wedge}\right)$ if $u-v \in \mathcal{K}_{\Theta}^{\infty, \gamma}\left(X^{\wedge}\right)$, and let $P=\mathcal{E}_{K}\left(X^{\wedge}\right) / \sim$ be the quotient space with respect to this equivalence relation. Then $P$ is called a continuous asymptotic type associated with the weight data $\boldsymbol{g}=(\gamma, \Theta)$. Observe that when $\widetilde{K} \in \mathcal{V}$ is another compact set, $\widetilde{K} \subset S_{(-\infty,(n+1) / 2-\gamma)}$, such that $K \cap S_{((n+1) / 2-\gamma+\vartheta, \infty)}=\widetilde{K} \cap S_{((n+1) / 2-\gamma+\vartheta, \infty)}$ we have $\mathcal{E}_{K}\left(X^{\wedge}\right) / \sim=\mathcal{E}_{\widetilde{K}}\left(X^{\wedge}\right) / \sim$, i.e., we get the same $P$. Let $\bar{\pi}_{\mathbb{C}} P$ denote the closure of the set $\pi_{\mathbb{C}} P:=K \cap S_{((n+1) / 2-\gamma+\vartheta, \infty)}$. Moreover, define $\operatorname{As}(X, \boldsymbol{g})$ to be the set of all $P$, associated with the weight data $\boldsymbol{g}$.

Now we set

$$
\mathcal{K}_{P}^{s, \gamma}\left(X^{\wedge}\right)=\mathcal{K}_{\Theta}^{s, \gamma}\left(X^{\wedge}\right)+\mathcal{E}_{K}\left(X^{\wedge}\right)
$$

for any $K$ as before. This space is independent of the specific $K$. We endow (1.1.8) with the Fréchet topology of the non-direct sum, cf. [26].

To extend the definition of continuous asymptotics to the infinite weight interval $(-\infty, 0]$ we first observe that every $P^{\prime} \in \operatorname{As}\left(X, \boldsymbol{g}^{\prime}\right)$ for $g^{\prime}=\left(\gamma,\left(\vartheta^{\prime}, 0\right]\right)$ induces an element $P \in \operatorname{As}(X, \boldsymbol{g})$ for $\boldsymbol{g}=(\gamma,(\vartheta, 0])$ whenever $\vartheta^{\prime} \leq \vartheta$, and there is then a canonical continuous embedding $\mathcal{K}_{P^{\prime}}^{s, \gamma}\left(X^{\wedge}\right) \hookrightarrow \mathcal{K}_{P}^{s, \gamma}\left(X^{\wedge}\right)$.

Given an element $V \in \mathcal{V}, V \subset S_{(-\infty,(n+1) / 2-\gamma)}$ we can form a sequence $V_{k}:=V \cap S_{\left((n+1) / 2-\gamma+\vartheta_{k}, \infty\right)}, k \in \mathbb{N}$, for any sequence $\left(\vartheta_{k}\right)_{k \in \mathbb{N}}$ such that $\vartheta_{k+1} \leq$ 
$\vartheta_{k}<0$ for all $k$ and $\vartheta_{k} \rightarrow-\infty$ as $k \rightarrow \infty$. Let $\boldsymbol{g}_{k}:=\left(\gamma,\left(\vartheta_{k}, 0\right]\right)$, and denote by $P_{k} \in \operatorname{As}\left(X, \boldsymbol{g}_{k}\right)$ the asymptotic type associated with $V_{k}$ and $\boldsymbol{g}_{k}$. We then have continuous embeddings $\mathcal{K}_{P_{k+1}}^{s, \gamma}\left(X^{\wedge}\right) \hookrightarrow \mathcal{K}_{P_{k}}^{s, \gamma}\left(X^{\wedge}\right)$ for all $k$, and we set

$$
\mathcal{K}_{P}^{s, \gamma}\left(X^{\wedge}\right)=\lim _{k \in \mathbb{N}} \mathcal{K}_{P_{k}}^{s, \gamma}\left(X^{\wedge}\right)
$$

Here, $P$ stands for the equivalence class of sequences $P_{k} \in \operatorname{As}\left(X, \boldsymbol{g}_{k}\right), k \in$ $\mathbb{N}$. Equivalence to another sequence $\widetilde{P}_{k} \in \operatorname{As}\left(X, \widetilde{\boldsymbol{g}}_{k}\right), \widetilde{\boldsymbol{g}}_{k}:=\left(\gamma,\left(\widetilde{\vartheta}_{k}, 0\right]\right)$, of analogous structure means that $\left(\widetilde{P}_{k}\right)_{k \in \mathbb{N}}$ comes from the same $V$ as $\left(P_{k}\right)_{k \in \mathbb{N}}$. It can easily be verified that (1.1.9) only depends on the equivalence class of such a sequence. Let $\operatorname{As}(X,(\gamma,(-\infty, 0])$ denote the set of all $P$ arising in this way. Let us set $V=\pi_{\mathbb{C}} P$ for the set $V \in \mathcal{V}$ associated with $P$. The trivial asymptotic type $\Theta$ characterised by $\pi_{\mathbb{C}} \Theta=\emptyset$ belongs to $\operatorname{As}(X, \boldsymbol{g})$ also in the set-up of continuous asymptotics.

Observe that for $P \in \operatorname{As}(X, \boldsymbol{g}), \boldsymbol{g}=(\gamma, \Theta), \Theta$ finite, an element $u \in$ $\mathcal{K}^{s, \gamma}\left(X^{\wedge}\right)$ belongs to $\mathcal{K}_{P}^{s, \gamma}\left(X^{\wedge}\right)$ if and only if for any compact $K \in \mathcal{V}$ such that $K \cap S_{((n+1) / 2-\gamma+\vartheta, \infty)}=\pi_{\mathbb{C}} P$ there is a $\zeta \in \mathcal{A}^{\prime}\left(K, C^{\infty}(X)\right)$ such that $u(r, x)-\omega(r)\left\langle\zeta, r^{-z}\right\rangle \in \mathcal{K}_{\Theta}^{s, \gamma}\left(X^{\wedge}\right)$. For $\Theta=(-\infty, 0]$ the property $u \in \mathcal{K}_{P}^{s, \gamma}\left(X^{\wedge}\right)$ is equivalent to the existence of a sequence $\zeta_{j} \in \mathcal{A}^{\prime}\left(K_{j}, C^{\infty}(X)\right), j \in \mathbb{N}$, for compact sets $K_{j} \subset \mathbb{C}, j \in \mathbb{N}$, $\sup \left\{\operatorname{Re} z: z \in K_{j}\right\} \rightarrow-\infty$ as $j \rightarrow \infty$, where $\bigcup_{j \in \mathbb{N}} K_{j}=\pi_{\mathbb{C}} P$, such that to every $\beta>0$ there is an $M(\beta)$ such that

$$
u(r, x)-\omega(r) \sum_{j=0}^{M}\left\langle\zeta_{j}, r^{-z}\right\rangle \in \mathcal{K}^{s, \gamma+\beta}\left(X^{\wedge}\right)
$$

for all $M \geq M(\beta)$ and any cut-off function $\omega$.

Let $P \in \operatorname{As}(X, \boldsymbol{g})\left(\right.$ or $\left.\in \operatorname{As}\left(X, \boldsymbol{g}^{\bullet}\right)\right)$ for $\boldsymbol{g}=(\gamma, \Theta)$, and set

$$
\mathcal{S}_{P}^{\gamma}\left(X^{\wedge}\right):=[\omega] \mathcal{K}_{P}^{\infty, \gamma}\left(X^{\wedge}\right)+[1-\omega] \mathcal{S}\left(\overline{\mathbb{R}}_{+}, C^{\infty}(X)\right)
$$

in the Fréchet topology of the non-direct sum. Here, use the fact that for every $P$ there is a sequence of Hilbert spaces $E^{j}, j \in \mathbb{N}$, with continuous embeddings $E^{j+1} \hookrightarrow E^{j} \hookrightarrow E^{0}=\mathcal{K}^{s, \gamma}\left(X^{\wedge}\right)$ for all $j$, such that

$$
\mathcal{S}_{P}^{\gamma}\left(X^{\wedge}\right)=\varliminf_{j \in \mathbb{N}} E^{j}
$$

and $\left(E_{j}\right)_{j \in \mathbb{N}}$ can be chosen in such a way that $\left\{\kappa_{\lambda}\right\}_{\lambda \in \mathbb{R}_{+}}$on $\mathcal{K}^{s, \gamma}\left(X^{\wedge}\right)$ restricts to a group action on each $E^{j}, j \in \mathbb{N}$. This allows us to form symbols taking values in spaces $(1.1 .12)$ 
In the following, for abbreviation we will write " $P \in \operatorname{As}\left(X, g^{(\bullet)}\right)$ " instead of " $P \in \operatorname{As}(X, \boldsymbol{g})$ or $P \in \operatorname{As}\left(X, g^{\bullet}\right)$ ".

Applying the general notation in connection with operator-valued symbols we then get the spaces

$$
S_{\mathrm{cl}}^{\mu}\left(U \times \mathbb{R}^{q} ; E, \widetilde{E}\right)
$$

for

$$
E:=\mathcal{K}^{s, \gamma}\left(X^{\wedge}\right) \oplus \mathbb{C}^{j_{-}}, \quad \widetilde{E}:=\mathcal{S}_{P}^{\gamma-\mu}\left(X^{\wedge}\right) \oplus \mathbb{C}^{j_{+}},
$$

$P \in \operatorname{As}\left(X, \boldsymbol{k}^{(\bullet)}\right), \boldsymbol{k}=(\gamma-\mu, \Theta)$, as well as

$$
S_{\mathrm{cl}}^{\mu}\left(U \times \mathbb{R}^{q} ; F, \widetilde{F}\right)
$$

for

$$
F:=\mathcal{K}^{s,-\gamma+\mu}\left(X^{\wedge}\right) \oplus \mathbb{C}^{j_{+}}, \quad \widetilde{F}:=\mathcal{S}_{Q}^{-\gamma}\left(X^{\wedge}\right) \oplus \mathbb{C}^{j_{-}},
$$

$Q \in \operatorname{As}\left(X, \boldsymbol{l}^{(\bullet)}\right), \boldsymbol{l}=(-\gamma, \Theta)$.

Incidentally, it will be convenient to assume asymptotic types $P$ to fulfill the "shadow condition". By this we understand the property $p \in \pi_{\mathbb{C}} P \Rightarrow$ $p-j \in \pi_{\mathbb{C}} P$ for all $j \in \mathbb{R}$ such that $p-j \in S_{((n+1) / 2-\gamma+\vartheta,(n+1) / 2-\gamma)}$ (where $P$ is associated with the weight data $(\gamma,(\vartheta, 0]))$.

Definition 1.1.1. An element

$$
g(y, \eta) \in \bigcap_{s \in \mathbb{R}} C^{\infty}\left(U \times \mathbb{R}^{q}, \mathcal{L}\left(\mathcal{K}^{s, \gamma}\left(X^{\wedge}\right) \oplus \mathbb{C}^{j_{-}}, \mathcal{K}^{\infty, \gamma-\mu}\left(X^{\wedge}\right) \oplus \mathbb{C}^{j_{+}}\right)\right)
$$

is said to be a Green symbol with asymptotics of type $(P, Q) \in \operatorname{As}\left(X, \boldsymbol{k}^{(\bullet)}\right) \times$ $\operatorname{As}\left(X, \boldsymbol{l}^{(\bullet)}\right)$ if $g(y, \eta)$ belongs to the space (1.1.13) with respect to $\operatorname{diag}\left(\kappa_{\lambda}, \varphi_{\lambda}\right)$ in the spaces, cf. formula (1.1.5), (1.1.14) and $g^{*}(y, \eta)$ belongs to (1.1.15) with respect to $\operatorname{diag}\left(\kappa_{\lambda}, \varphi_{\lambda}^{-1}\right)$ in the spaces $(1.1 .16)$ for all $s \in \mathbb{R}$, where $*$ denotes the $(y, \eta)$-wise formal adjoint.

The formal adjoint is given by $(g u, v)_{\mathcal{K}^{0,0}\left(X^{\wedge}\right) \oplus \mathbb{C}^{j_{+}}}=\left(u, g^{*} v\right)_{\mathcal{K}^{0,0}\left(X^{\wedge}\right) \oplus \mathbb{C}^{j_{-}}}$ for all $u \in C_{0}^{\infty}\left(X^{\wedge}\right) \oplus \mathbb{C}^{j_{-}}, v \in C_{0}^{\infty}\left(X^{\wedge}\right) \oplus \mathbb{C}^{j_{+}}$. Let $\mathcal{R}_{G}^{\mu}\left(U \times \mathbb{R}^{q}, \boldsymbol{g} ; j_{-}, j_{+}\right)_{P, Q}$ for $\boldsymbol{g}=(\gamma, \gamma-\mu, \Theta)$ denote the space of all those $g(y, \eta)$. For $j_{-}=j_{+}=0$ we simply write $R_{G}^{\mu}\left(U \times \mathbb{R}^{q}, \boldsymbol{g}\right)$. Moreover, $\mathcal{R}_{G}^{\mu}\left(U \times \mathbb{R}^{q}, \boldsymbol{g} ; j_{-}, j_{+}\right)$denotes the union of all spaces $\mathcal{R}_{G}^{\mu}\left(U \times \mathbb{R}^{q}, \boldsymbol{g} ; j_{-}, j_{+}\right)_{P, Q}$ over $P \in \operatorname{As}(X, \boldsymbol{k}), Q \in \operatorname{As}(X, \boldsymbol{l})$; analogous notation is used with $\boldsymbol{g}^{\bullet}$ when the union refers to discrete asymptotic types. 
For purposes below we introduce the subspace of all flat elements, denoted by

$$
\mathcal{R}_{G}^{\mu}\left(U \times \mathbb{R}^{q}, \boldsymbol{g} ; \boldsymbol{w}\right)_{\mathcal{O}}
$$

where the asymptotic types $P \in \operatorname{As}(X, \boldsymbol{k})$ and $Q \in \operatorname{As}(X, \boldsymbol{l})$, are trivial, while $\boldsymbol{w}:=\left(e, f ; j_{-}, j_{+}\right)$are simply dimension data with the meaning that upper left corners are $f \times e$-matrices, while $j_{-}$is the number of trace entries, $j_{+}$is the number of potential entries in the corresponding block matrices. Similarly, to generalise the above-mentioned notation to the $f \times e$-matrix-valued case, we have the spaces

$$
\mathcal{R}_{G}^{\mu}\left(U \times \mathbb{R}^{q}, \boldsymbol{g} ; \boldsymbol{w}\right)_{P, Q}
$$

for arbitrary $P \in \operatorname{As}(X, \boldsymbol{k})$ and $Q \in \operatorname{As}(X, \boldsymbol{l})$.

\section{$\S 1.2$. Mellin edge symbols}

We now briefly formulate other essential ingredients of the edge symbol calculus. First we look at smoothing Mellin operators with asymptotics.

Consider the space $L_{\mathrm{cl}}^{\mu}\left(X ; \mathbb{R}^{l}\right)$ (cf. the notation in Section 1$)$ for $l=1$, and identify $\mathbb{R}$ with a weight line $\Gamma_{\beta}=\{z: \operatorname{Re} z=\beta\}$ for any fixed real $\beta$ via the bijection $\Gamma_{\beta} \rightarrow \mathbb{R}, \quad z \rightarrow \operatorname{Im} z$. We then set $L_{\mathrm{cl}}^{\mu}\left(X ; \Gamma_{\beta}\right):=\{f(z): f(\beta+i \tau) \in$ $\left.L_{\mathrm{cl}}^{\mu}\left(X ; \mathbb{R}_{\tau}\right)\right\}$. The spaces $L_{\mathrm{cl}}^{\mu}\left(X ; \mathbb{R}^{l}\right)$ are Fréchet in a natural way; in particular, $L_{\mathrm{cl}}^{\mu}\left(X ; \Gamma_{\beta}\right)$ is a Fréchet space.

Given an element $f\left(r, r^{\prime}, z\right) \in C^{\infty}\left(\overline{\mathbb{R}}_{+} \times \overline{\mathbb{R}}_{+}, L_{\text {cl }}^{\mu}\left(X ; \Gamma_{1 / 2-\gamma}\right)\right)$ we set

$$
\begin{aligned}
\operatorname{op}_{M}^{\gamma}(f) u(r) & =M_{\gamma, z \rightarrow r}^{-1}\left\{M_{\gamma, r^{\prime} \rightarrow z} f\left(r, r^{\prime}, z\right) u\left(r^{\prime}\right)\right\} \\
& =\frac{1}{2 \pi i} \int_{\Gamma_{\frac{1}{2}-\gamma}} \int_{0}^{\infty}\left(\frac{r}{r^{\prime}}\right)^{-z} f\left(r, r^{\prime}, z\right) u\left(r^{\prime}\right) \frac{d r^{\prime}}{r^{\prime}} d z
\end{aligned}
$$

first defined for $u \in C_{0}^{\infty}\left(\mathbb{R}_{+}, C^{\infty}(X)\right)$ and then extended to weighted Sobolev spaces. The right hand side makes sense as an oscillatory integral. For $\gamma=0$ we also write $\operatorname{op}_{M}(\cdot)$ instead of $\operatorname{op}_{M}^{0}(\cdot)$. Note that op ${ }_{M}^{\gamma}(f)=r^{\gamma} \mathrm{op}_{M}\left(T^{-\gamma} f\right) r^{-\gamma}$ for $\left(T^{-\gamma} f\right)(z)=f(z-\gamma)$.

Let $\mathbf{A s}^{\bullet}(X)$ denote the set of all sequences

$$
R=\left\{\left(r_{j}, n_{j}, G_{j}\right)\right\}_{j \in \mathbb{Z}}
$$

such that $\pi_{\mathbb{C}} R:=\left\{r_{j}\right\}_{j \in \mathbb{Z}} \subset \mathbb{C}$ intersects $S_{\left[c, c^{\prime}\right]}$ in a finite set for every $c \leq c^{\prime}$, $\operatorname{Re} r_{j} \rightarrow \mp \infty$ as $j \rightarrow \pm \infty, n_{j} \in \mathbb{N}$, and $G_{j} \subset L^{-\infty}(X)$ is a subspace of finite 
dimension (for $G_{j}=\{0\}$ we will ignore the corresponding triple in $R$; we admit $\pi_{\mathbb{C}} R$ to be finite or a set infinite on one side). The elements $R \in \mathbf{A s}^{\bullet}(X)$ are called discrete asymptotic types for Mellin symbols. Given a closed set $A \subset \mathbb{C}$, a function $\chi \in C^{\infty}(\mathbb{C})$ is said to be an $A$-excision function, if there are $0<\varepsilon_{0}<\varepsilon_{1}$ such that $\chi(z)=0$ for $\operatorname{dist}(z, A)<\varepsilon_{0}$ and $\chi(z)=1$ for $\operatorname{dist}(z, A)>\varepsilon_{1}$.

Define $M_{R}^{-\infty}(X)$ to be the space of all $f(z) \in \mathcal{A}\left(\mathbb{C} \backslash \pi_{\mathbb{C}} R, L^{-\infty}(X)\right)$ such that

$$
\left.\chi(z) f(z)\right|_{\Gamma_{\beta}} \in \mathcal{S}\left(\Gamma_{\beta}, L^{-\infty}(X)\right)
$$

for every $\pi_{\mathbb{C}} R$-excision function $\chi(z)$ and all $\beta \in \mathbb{R}$, uniformly in $c \leq \operatorname{Re} z \leq c^{\prime}$ for every $c \leq c^{\prime}$, and $f(z)$ is meromorphic with poles at all $r_{j} \in \pi_{\mathbb{C}} R$ of multiplicity $n_{j}+1$ and Laurent coefficients at $\left(z-r_{j}\right)^{-(k+1)}$ belonging to $G_{j}$, for all $0 \leq k \leq \kappa_{j}$ and all $j$. We consider the space $M_{R}^{-\infty}(X)$ in its natural Fréchet topology.

The elements $f \in M_{R}^{-\infty}(X), R \in \mathbf{A s}^{\bullet}(X)$, play the role of symbols of pseudo-differential operators on $\mathbb{R}_{+} \times X$ of the form op ${ }_{M}^{\beta}(f)$ for $\pi_{\mathbb{C}} R \cap \Gamma_{1 / 2-\beta}=$ $\emptyset$. If $\omega(r), \widetilde{\omega}(r)$ are cut-off functions, we get continuous operators

$$
\omega \operatorname{op}_{M}^{\gamma-\frac{n}{2}}(f) \widetilde{\omega}: \mathcal{K}_{P}^{s, \gamma}\left(X^{\wedge}\right) \rightarrow \mathcal{S}_{Q}^{\gamma}\left(X^{\wedge}\right)
$$

when $\pi_{\mathbb{C}} R \cap \Gamma_{(n+1) / 2-\gamma}=\emptyset$, for all $s \in \mathbb{R}$ and every $P \in \operatorname{As}\left(X, \boldsymbol{g}^{\bullet}\right), \boldsymbol{g}=(\gamma, \Theta)$, with some resulting $Q \in \operatorname{As}\left(X, \boldsymbol{g}^{\bullet}\right)$; here, $\Theta$ is an arbitrary weight interval.

Analogous relations are needed for continuous asymptotic types. Let us start from a compact set $K \in \mathcal{V}$, and choose a $\zeta \in \mathcal{A}^{\prime}\left(K, L^{-\infty}(X)\right)$. Then, setting $u(r)=\omega(r)\left\langle\zeta_{w}, r^{-w}\right\rangle$, we have $f_{K}(z):=M_{\delta} u(z) \in \mathcal{A}\left(\mathbb{C} \backslash K, L^{-\infty}(X)\right)$ for any $\delta \in \mathbb{R}$ such that $K \subset\{z: \operatorname{Re} z<1 / 2-\delta\}$, and $f_{K}$ is independent of the choice of $\delta$. This gives us special examples of symbols for Mellin pseudodifferential operators.

In general, let $V \in \mathcal{V}$ be arbitrary and set $V_{c, c^{\prime}}=V \cap S_{\left[c, c^{\prime}\right]}$ for $c \leq c^{\prime}$. Then $M_{V}^{-\infty}(X)$ denotes the subspace of all $f(z) \in \mathcal{A}\left(\mathbb{C} \backslash V, L^{-\infty}(X)\right)$ such that for every $c \leq c^{\prime}$ there is an element $\zeta_{c, c^{\prime}} \in \mathcal{A}^{\prime}\left(V_{c, c^{\prime}}, L^{-\infty}(X)\right)$ with the properties

$$
r_{c, c^{\prime}}(z):=f(z)-f_{V_{c, c^{\prime}}}(z) \in \mathcal{A}\left(S_{\left(c, c^{\prime}\right)}, L^{-\infty}(X)\right),
$$

and $\left.r_{c, c^{\prime}}(z)\right|_{\Gamma_{\beta}} \in \mathcal{S}\left(\Gamma_{\beta}, L^{-\infty}(X)\right)$ for every $c<\beta<c^{\prime}$, uniformly in $c+\varepsilon \leq$ $\beta \leq c^{\prime}-\varepsilon$ for every $\varepsilon>0$.

To indicate a relation between $V \in \mathcal{V}$ and asymptotic phenomena in the calculus below we identify $\mathcal{V}$ with a set $\mathbf{A s}(X)$ of so-called continuous asymptotic types $R$ for Mellin symbols. Although for the moment this is only another 
notation for $\mathcal{V}$, we keep in mind the connection with the range $L^{-\infty}(X)$ of analytic functionals; later on we will employ analogous relations for manifolds $X$ with edges and then integrate further non-trivial information from $X$.

In other words, instead of $M_{V}^{-\infty}(X)$ we now write $M_{R}^{-\infty}(X)$ for $R \in \mathbf{A s}(X)$ and set $V=: \pi_{\mathbb{C}} R$.

The space $M_{R}^{-\infty}(X)$ is Fréchet in a natural way. Note that for $S \in \mathbf{A} \mathbf{s}^{\bullet}(X)$ we have a continuous embedding $M_{S}^{-\infty}(X) \hookrightarrow M_{R}^{-\infty}(X)$ when $R \in \mathbf{A s}(X)$ is defined in terms of $W:=\pi_{\mathbb{C}} S$. Let $M_{\mathbf{A s}}^{-\infty}(X)\left(M_{\mathbf{A s}^{\bullet}}^{-\infty}(X)\right)$ denote the union of the spaces $M_{R}^{-\infty}(X)$ over all $R \in \mathbf{A s}(X)\left(\in \mathbf{A} \mathbf{s}^{\bullet}(X)\right)$ with the inductive limit topology.

Incidentally, to express a relation for discrete or continuous asymptotic types for Mellin symbols, we write $R \in \mathbf{A} \mathbf{s}^{(\bullet)}(X)$ if $R \in \mathbf{A} \mathbf{s}^{\bullet}(X)$ or $R \in \mathbf{A s}(X)$.

Given an element $f\left(r, r^{\prime}, z\right) \in C^{\infty}\left(\overline{\mathbb{R}}_{+} \times \overline{\mathbb{R}}_{+}, M_{R}^{-\infty}(X)\right)$ for $R \in \mathbf{A s}^{(\bullet)}(X)$, $\pi_{\mathbb{C}} R \cap \Gamma_{(n+1) / 2-\gamma}=\emptyset$, for every $P \in \operatorname{As}\left(X, \boldsymbol{g}^{(\bullet)}\right), \boldsymbol{g}=(\gamma, \Theta)$ there is a $Q \in$ $\operatorname{As}\left(X, \boldsymbol{g}^{(\bullet)}\right)$ such that (1.2.2) is a continuous operator for all $s \in \mathbb{R}$.

We now pass to a class of operator-valued symbols in $(y, \eta) \in U \times \mathbb{R}^{q}$, $U \subseteq \mathbb{R}^{p}$ open, with values in operators of the form (1.2.2). For notational convenience we give formulations for continuous asymptotics. The discrete case is completely analogous.

In the following constructions $\omega_{i}(r), i=0,1, \ldots$ will denote arbitrary cutoff functions. Moreover, let $\eta \rightarrow[\eta]$ be any function in $C^{\infty}\left(\mathbb{R}^{q}\right)$ such that $[\eta]>0$ for all $\eta \in \mathbb{R}^{q}$ and $[\eta]=|\eta|$ for all $|\eta| \geq c$ for some constant $c>0$.

Let

$$
R_{j \alpha}, Q_{j \alpha} \in \mathbf{A s}(X), \quad \pi_{\mathbb{C}} R_{j \alpha} \cap \Gamma_{\frac{n+1}{2}-\gamma_{j \alpha}}=\pi_{\mathbb{C}} Q_{j \alpha} \cap \Gamma_{\frac{n+1}{2}-\delta_{j \alpha}}=\emptyset
$$

for weights $\gamma_{j \alpha}, \delta_{j \alpha} \in \mathbb{R}, 0 \leq j \leq k$, where

$$
\gamma \geq \gamma_{j \alpha} \geq \gamma-j, \quad \gamma \geq \delta_{j \alpha} \geq \gamma-j, \quad 0 \leq j \leq k
$$

for some reference weight $\gamma \in \mathbb{R}$. Choose elements

$$
f_{j \alpha}(y) \in C^{\infty}\left(U, M_{R_{j \alpha}}^{-\infty}(X)\right), \quad h_{j \alpha}(y) \in C^{\infty}\left(U, M_{Q_{j \alpha}}^{-\infty}(X)\right)
$$

for $0 \leq j \leq k$ and $\alpha \in \mathbb{N}^{q},|\alpha| \leq j$. Here, $k \in \mathbb{N}$ is fixed and connected with the length of a weight interval $\Theta=(-(k+1), 0]$.

We then form the operator functions

$$
m_{j \alpha}(y, \eta):=r^{-\mu} \omega_{0}(r[\eta]) r^{j}\left\{\operatorname{op}_{M}^{\gamma_{j \alpha}-\frac{n}{2}}\left(f_{j \alpha}\right)(y)+\operatorname{op}_{M}^{\delta_{j \alpha}-\frac{n}{2}}\left(h_{j \alpha}\right)(y)\right\} \eta^{\alpha} \omega_{1}(r[\eta])
$$


that are $C^{\infty}$ in $(y, \eta) \in U \times \mathbb{R}^{q}$ with values in the space of continuous operators $\mathcal{K}^{s, \gamma}\left(X^{\wedge}\right) \rightarrow \mathcal{K}^{\infty, \gamma-\mu}\left(X^{\wedge}\right)$, for all $s \in \mathbb{R}$ and all $j$.

By virtue of $m_{j \alpha}(y, \lambda \eta)=\lambda^{\mu-j+|\alpha|} \kappa_{\lambda} m_{j \alpha}(y, \eta) \kappa_{\lambda}^{-1}$, for all $\lambda \geq 1,|\eta| \geq c$, we have $m_{j \alpha}(y, \eta) \in S_{\mathrm{cl}}^{\mu-j+|\alpha|}\left(U \times \mathbb{R}^{q} ; \mathcal{K}^{s, \gamma}\left(X^{\wedge}\right), \mathcal{K}^{\infty, \gamma-\mu}\left(X^{\wedge}\right)\right)$ for every $s \in \mathbb{R}$.

Given weight data $\boldsymbol{g}=(\gamma, \gamma-\mu, \Theta)$ for $\gamma, \mu \in \mathbb{R}$ and $\Theta=(-(k+1), 0]$, $k \in \mathbb{N}$, we define $R_{M+G}^{\mu}\left(U \times \mathbb{R}^{q}, \boldsymbol{g}\right)$ to be the space of all operator functions $m(y, \eta)+g(y, \eta)$ for arbitrary $g(y, \eta) \in R_{G}^{\mu}\left(U \times \mathbb{R}^{q}, \boldsymbol{g}\right)$ and

$$
m(y, \eta)=\sum_{j=0}^{k} \sum_{|\alpha| \leq j} m_{j \alpha}(y, \eta)
$$

for $m_{j \alpha}(y, \eta)$ of the form $(1.2 .6)$ with arbitrary $f_{j \alpha}(y), h_{j \alpha}(y)$ and $\gamma_{j \alpha}, \delta_{j \alpha}$ defined by (1.2.5), (1.2.3) and (1.2.4).

Given an element $(m+g)(y, \eta) \in R_{M+G}^{\mu}\left(U \times \mathbb{R}^{q}, \boldsymbol{g}\right)$, where $m(y, \eta)$ has the form (1.2.7) with summands (1.2.6) and $g(y, \eta) \in R_{G}^{\mu}\left(U \times \mathbb{R}^{q}, \boldsymbol{g}\right), \boldsymbol{g}=$ $(\gamma, \gamma-\mu, \Theta), \Theta=(-(k+1), 0]$, we set

$$
\sigma_{M}^{(j)}(m+g)(y, z, \eta):=\sum_{|\alpha| \leq j}\left(f_{j \alpha}(y, z)+h_{j \alpha}(y, z)\right) \eta^{\alpha}
$$

and $\sigma_{(M)}(m+g):=\left(\sigma_{M}^{(j)}(m+g)\right)_{0 \leq j \leq k}$. Let $\mathcal{P}^{j}(\eta)$ denote the space of all polynomials of degree $j$ in $\eta=\left(\eta_{1}, \ldots, \eta_{q}\right)$. Then $\sigma_{(M)}$ is a linear map

$$
\sigma_{(M)}: R_{M+G}^{\mu}\left(U \times \mathbb{R}^{q}, \boldsymbol{g}\right) \rightarrow \bigoplus_{j=0}^{k} C^{\infty}\left(U, M_{\mathbf{A s}}^{-\infty}(X)\right) \otimes \mathcal{P}^{j}(\eta) .
$$

Remark 1.2.1. The map (1.2.9) is well-defined, i.e., independent of the specific choice of decompositions $f_{j \alpha}+h_{j \alpha}$, weights $\gamma_{j \alpha}, \delta_{j \alpha}$ in (1.2.6), cut-off functions $\omega_{0}, \omega_{1}$ and of the function $[\eta]$. We have $\operatorname{ker} \sigma_{(M)}=R_{G}^{\mu}\left(U \times \mathbb{R}^{q}, \boldsymbol{g}\right)$.

Thus, without loss of generality we may (and will) normalise the choice of weights $\gamma_{j \alpha}$ and $\delta_{j \alpha}$ in (1.2.5) by setting

$$
\gamma_{j \alpha}:=\gamma-\frac{1}{3}, \quad \delta_{j \alpha}:=\gamma-\frac{2}{3}
$$

for $0 \leq|\alpha| \leq j, 1 \leq j \leq k$. Moreover, we fix the cut-off functions $\omega_{0}$ and $\omega_{1}$ as well as the function $[\eta]$. Furthermore, to single out convenient spaces of Mellin + Green symbols we form a Mellin asymptotic type $R \in \mathbf{A s}(X)$ by setting $V=\pi_{\mathbb{C}} R=\bigcup_{\substack{0 \leq|\alpha| \leq j \\ 1 \leq j \leq k}}\left(\pi_{\mathbb{C}} P_{j \alpha} \cup \pi_{\mathbb{C}} Q_{j \alpha}\right)$ when $m$ is given in terms of $m_{j \alpha}$ by 
(1.2.7). Applying a Cousin decomposition argument we find asymptotic types $R_{0}, R_{1}, R_{2} \in \mathbf{A s}(X)$ such that $\pi_{\mathbb{C}} R_{i} \subseteq \pi_{\mathbb{C}} R$ for $0 \leq i \leq 2$ and

$$
\pi_{\mathbb{C}} R_{0} \cap \Gamma_{\frac{n+1}{2}-\gamma}=\pi_{\mathbb{C}} R_{1} \cap \Gamma_{\frac{n+1}{2}-\left(\gamma-\frac{1}{3}\right)}=\pi_{\mathbb{C}} R_{2} \cap \Gamma_{\frac{n+1}{2}-\left(\gamma-\frac{2}{3}\right)}=\emptyset
$$

and elements $f_{0}(y, z) \in C^{\infty}\left(U, M_{R_{0}}^{-\infty}(X)\right), f_{j \alpha, i}(y, z) \in C^{\infty}\left(U, M_{R_{i}}^{-\infty}(X)\right), i=$ 1,2 , such that

$$
\begin{aligned}
\sigma_{M}^{(0)}(m)(y, z) & =f_{0}(y, z), \\
\sigma_{M}^{(j)}(y, z, \eta) & =\sum_{|\alpha| \leq j}\left(f_{j \alpha, 1}(y, z)+f_{j \alpha, 2}(y, z)\right) \eta^{\alpha} .
\end{aligned}
$$

Now, if we start from $R \in \mathbf{A s}(X)$ where $\pi_{\mathbb{C}} R=\pi_{\mathbb{C}} R_{1} \cup \pi_{\mathbb{C}} R_{2}$ and prescribe $R_{0} \in \mathbf{A s}(X)$, to every tuple of elements

$$
f_{0} \in C^{\infty}\left(U, M_{R_{0}}^{-\infty}(X)\right), \quad f_{j \alpha}(y, z) \in C^{\infty}\left(U, M_{R}^{-\infty}(X)\right)
$$

$|\alpha| \leq j, 1 \leq j \leq k$, we find decompositions $f_{j \alpha}=f_{j \alpha, 1}+f_{j \alpha, 2}$, where $f_{j \alpha, i} \in$ $C^{\infty}\left(U, M_{R_{i}}^{-\infty}(X)\right), i=1,2$. Setting

$$
\begin{array}{r}
m(y, \eta)=r^{-\mu} \omega_{0}(r[\eta]) \operatorname{op}_{M}^{\gamma-\frac{n}{2}}\left(f_{0}\right)(y) \omega_{1}(r[\eta])+r^{-\mu} \sum_{j=0}^{k} r^{j} \sum_{|\alpha| \leq j} \omega_{0}(r[\eta]) \\
\left\{\operatorname{op}_{M}^{\gamma-\frac{1}{3}-\frac{n}{2}}\left(f_{j \alpha, 1}\right)(y)+\mathrm{op}_{M}^{\gamma-\frac{2}{3}-\frac{n}{2}}\left(f_{j \alpha, 2}\right)(y)\right\} \eta^{\alpha} \omega_{1}(r[\eta])
\end{array}
$$

we have

$$
\sigma_{(M)}(m)=\left\{f_{0}(y, z),\left(\sum_{|\alpha| \leq j} f_{j \alpha}(y, z) \eta^{\alpha}\right)_{1 \leq j \leq k}\right\} .
$$

It can easily be proved that there are asymptotic types $P \in \operatorname{As}(X,(\gamma-\mu, \Theta))$, $Q \in \operatorname{As}(X,(-\gamma, \Theta))$ such that when we take $\sigma_{(M)}(m)$ and choose any other decomposition $f_{j \alpha}=\tilde{f}_{j \alpha, 1}+\widetilde{f}_{j \alpha, 2}$ of analogous kind and form $\tilde{m}(y, \eta)$ similarly to $(1.2 .11)$, we have

$$
m(y, \eta)-\widetilde{m}(y, \eta) \in R_{G}^{\mu}\left(U \times \mathbb{R}^{q}, \boldsymbol{g}\right)_{P, Q}
$$

To admit more general Green summands $g(y, \eta)$ in expressions $(m+g)(y, \eta)$ we choose $\pi_{\mathbb{C}} P$ and $\pi_{\mathbb{C}} Q$ so large that we also have $g(y, \eta) \in R_{G}^{\mu}\left(U \times \mathbb{R}^{q}, \boldsymbol{g}\right)_{P, Q}$. If those $P, Q$ contain the minimal ones in the sense of relation (1.2.12), we call the 
asymptotic types $\left(R_{1}, R_{2} ; P, Q\right)$ compatible. It is obvious that every element $a(y, \eta) \in R_{M+G}^{\mu}\left(U \times \mathbb{R}^{q}, \boldsymbol{g}\right)$ is contained in a sum of vector spaces

$$
R_{M+G}^{\mu}\left(U \times \mathbb{R}^{q}, \boldsymbol{g}\right)_{1}+R_{M+G}^{\mu}\left(U \times \mathbb{R}^{q}, \boldsymbol{g}\right)_{2},
$$

where $R_{M+G}^{\mu}\left(U \times \mathbb{R}^{q}, \boldsymbol{g}\right)_{i}$ is defined to be the set of all $m_{i}(y, \eta)+g(y, \eta)$ with $\sigma_{(M)}\left(m_{i}\right) \in C^{\infty}\left(U, M_{R_{0}}^{-\infty}(X)\right) \oplus \bigoplus_{j=1}^{k} C^{\infty}\left(U, M_{R_{i}}^{-\infty}(X)\right) \otimes \mathcal{P}^{j}(\eta)$ and $g(y, \eta) \in$ $R_{G}^{\mu}\left(U \times \mathbb{R}^{q}, \boldsymbol{g}\right)_{P, Q}$, for a suitable choice of asymptotic types $\left(R_{0}, R_{1}, R_{2} ; P, Q\right)$ with compatible $\left(R_{1}, R_{2} ; P, Q\right)$. To have a notation for the considerations below we define $\mathbf{A s}_{M+G}(X, \boldsymbol{g})$ to be the set of all such tuples $S:=\left(R_{0}, R_{1}, R_{2} ; P, Q\right)$ and denote by $\mathcal{R}_{M+G}^{\mu}\left(U \times \mathbb{R}^{q}, \boldsymbol{g}\right)_{S}$ the space (1.2.13).

Let $\mathcal{R}_{M+G}^{\mu}\left(U \times \mathbb{R}^{q} ; \boldsymbol{g} ; j_{-}, j_{+}\right)$denote the space of all $2 \times 2$-block matrix operator functions of the form $\left(\begin{array}{cc}m & 0 \\ 0 & 0\end{array}\right)(y, \eta)+g(y, \eta)$, where $m(y, \eta) \in \mathcal{R}_{M+G}^{\mu}(U$ $\left.\times \mathbb{R}^{q}, \boldsymbol{g}\right)$ and $g(y, \eta) \in \mathcal{R}_{G}^{\mu}\left(U \times \mathbb{R}^{q}, \boldsymbol{g} ; j_{-}, j_{+}\right)$.

Let us set $\mathcal{S}^{\gamma}\left(X^{\wedge}\right):=[\omega] \mathcal{K}^{\infty, \gamma}\left(X^{\wedge}\right)+[1-\omega] \mathcal{S}\left(\overline{\mathbb{R}}_{+}, C^{\infty}(X)\right)$ for any $P \in$ $\operatorname{As}(X,(\gamma, \Theta))$.

Theorem 1.2.2. $\quad a(y, \eta) \in \mathcal{R}_{M+G}^{\mu}\left(U \times \mathbb{R}^{q}, \boldsymbol{g} ; j_{-}, j_{+}\right)$for $\boldsymbol{g}=(\gamma, \gamma-$ $\mu, \Theta), \Theta=(-(k+1), 0]$, implies

$$
a(y, \eta) \in S_{\mathrm{cl}}^{\mu}\left(U \times \mathbb{R}^{q} ; E, \widetilde{E}\right)
$$

for $E=\mathcal{K}^{s, \gamma}\left(X^{\wedge}\right) \oplus \mathbb{C}^{j_{-}}, \widetilde{E}=\mathcal{S}^{\gamma}\left(X^{\wedge}\right) \oplus \mathbb{C}^{j_{+}}, s \in \mathbb{R}$, as well as for $E=$ $\mathcal{K}_{P}^{s, \gamma}\left(X^{\wedge}\right) \oplus \mathbb{C}^{j_{-}}, \widetilde{E}=\mathcal{S}_{Q}^{\gamma-\mu}\left(X^{\wedge}\right) \oplus \mathbb{C}^{j_{+}}, s \in \mathbb{R}$, for every $P \in \operatorname{As}(X,(\gamma, \Theta))$ with some resulting $Q \in \operatorname{As}(X,(\gamma-\mu, \Theta))$, dependent on $P$ and on the asymptotic data of the symbol a (contributed by the Mellin and Green summands) but not on $s$.

For the Green summand the assertion is part of Definition 1.1.1, while for the Mellin operator family we have $(y, \eta)$-wise the desired mapping properties, with smoothness in $(y, \eta)$, and the summands in (1.2.7) are $\kappa_{\lambda}$-homogeneous of degree $\mu-j+|\alpha|$ in $\eta$ for large $|\eta|$ which entails the result for Mellin terms.

\section{$\S 1.3$. The edge symbol algebra}

The parameter-dependent edge calculus relies on edge-degenerate symbols in local wedge coordinates $(r, x, y) \in \mathbb{R}_{+} \times \Sigma \times \Omega$ for open $\Sigma \subseteq \mathbb{R}^{n}, \Omega \subseteq \mathbb{R}^{q}$, with covariables $(\varrho, \xi, \eta) \in \mathbb{R}^{1+n+q}$ and a parameter $\lambda \in \mathbb{R}^{l}$. Since symbols make sense for arbitrary dimensions of variables and covariables, we first replace $\Omega$ by an open set $U \subseteq \mathbb{R}^{p}$, admit $t:=\left(r, r^{\prime}\right) \in\left(\mathbb{R}_{+}\right)^{2}$ and omit $\lambda$. Later on, if parameters play a role, instead of $\eta$ we write $(\eta, \lambda)$, i.e., replace $q$ by $q+l$. 
Let $S_{\mathrm{cl}}^{\mu}\left(\left(\overline{\mathbb{R}}_{+}\right)^{2} \times \Sigma \times U \times \mathbb{R}^{1+n+q}\right)_{\text {edge }}$ denote the subspace of all

$$
p(t, x, y, \varrho, \xi, \eta) \in S_{\mathrm{cl}}^{\mu}\left(\left(\mathbb{R}_{+}\right)^{2} \times \Sigma \times U \times \mathbb{R}^{1+n+q}\right)
$$

such that there is a $\tilde{p}(t, x, y, \varrho, \xi, \eta) \in S_{\mathrm{cl}}^{\mu}\left(\left(\overline{\mathbb{R}}_{+}\right)^{2} \times \Sigma \times U \times \mathbb{R}^{1+n+q}\right)$ for which $p(t, x, y, \varrho, \xi, \eta)=\widetilde{p}(t, x, y, r \varrho, \xi, r \eta)$.

Let $\left\{G_{j}\right\}_{1 \leq j \leq N}$ be a covering of $X$ by coordinate neighbourhoods, let $\chi_{j}: G_{j} \rightarrow \Sigma$ be charts, $\left\{\varphi_{j}\right\}_{1 \leq j \leq N}$ a subordinate partition of unity, and $\left\{\psi_{j}\right\}_{1 \leq j \leq N}$ another system of functions $\psi_{j} \in C_{0}^{\infty}\left(G_{j}\right)$ such that $\varphi_{j} \psi_{j}=\varphi_{j}$ for all $j$.

Given symbols

$$
p_{j}(t, x, y, \varrho, \xi, \eta) \in S_{\mathrm{cl}}^{\mu}\left(\left(\overline{\mathbb{R}}_{+}\right)^{2} \times \Sigma \times U \times \mathbb{R}^{1+n+q}\right)_{\text {edge }}, \quad 1 \leq j \leq N
$$

we can form $(t, y, \varrho, \eta)$-depending operator families

$$
p(t, y, \varrho, \eta):=\sum_{j=1}^{N} \varphi_{j}\left(\chi_{j}^{-1}\right)_{*} \mathrm{op}_{x}\left(p_{j}\right)(t, y, \varrho, \eta) \psi_{j} .
$$

Here $\operatorname{op}_{x}(a) u(x)=\iint e^{i\left(x-x^{\prime}\right) \xi} a(x, \xi) u\left(x^{\prime}\right) d x^{\prime} d \xi$, when $a$ is any symbol in $(x, \xi)$.

Set $L^{-\infty}\left(X ;\left(\mathbb{R}_{+}\right)^{2} \times U \times \mathbb{R}^{1+q}\right):=\mathcal{S}\left(\mathbb{R}^{1+q}, C^{\infty}\left(\left(\mathbb{R}_{+}\right)^{2} \times U, L^{-\infty}(X)\right)\right)$, and let $L_{\mathrm{cl}}^{\mu}\left(X ;\left(\overline{\mathbb{R}}_{+}\right)^{2} \times U \times \mathbb{R}^{1+q}\right)$ edge denote the space of all operator functions of the form $p(t, y, \varrho, \eta)+c(t, y, \varrho, \eta)$, where $p$ is given by (1.3.2) for arbitrary symbols (1.3.1) and $c \in L^{-\infty}\left(X ;\left(\mathbb{R}_{+}\right)^{2} \times U \times \mathbb{R}^{1+q}\right)$.

From $a\left(r, r^{\prime}, y, \varrho, \eta\right) \in L_{\mathrm{cl}}^{\mu}\left(X ;\left(\overline{\mathbb{R}}_{+}\right)^{2} \times U \times \mathbb{R}^{1+q}\right)_{\text {edge }}$ we can pass to $\operatorname{op}_{r}(a)(y, \eta)\left(\operatorname{op}_{r}(\cdot)\right.$ is the pseudo-differential action on $\mathbb{R}_{+}$with respect to the Fourier transform on $\mathbb{R})$ and get $\operatorname{op}_{r}(a)(y, \eta) \in C^{\infty}\left(U, L_{\mathrm{cl}}^{\mu}\left(X^{\wedge} ; \mathbb{R}^{q}\right)\right)$.

To organise a pseudo-differential algebra on the wedge $\mathbb{R}_{+} \times X \times \Omega$ with edge-degenerate symbols (1.3.1) such that the associated operators act in weighted Sobolev spaces and respect asymptotics we will modify the families (1.3.2) by smoothing elements of the class $L^{-\infty}\left(X ;\left(\mathbb{R}_{+}\right)^{2} \times U \times \mathbb{R}^{1+q}\right)$ such that the resulting new families have a holomorphic extension to the complex plane with respect to $\varrho$.

To this end we introduce the space $M_{\mathcal{O}}^{\mu}\left(X ; \mathbb{R}^{q}\right)$ of all functions $h(z, \eta) \in$ $\mathcal{A}\left(\mathbb{C}, L_{\mathrm{cl}}^{\mu}\left(X ; \mathbb{R}^{q}\right)\right)$ that have the property $\left.h(z, \eta)\right|_{\Gamma_{\beta}} \in L_{\mathrm{cl}}^{\mu}\left(X ; \Gamma_{\beta} \times \mathbb{R}^{q}\right)$ for each $\beta \in \mathbb{R}$, uniformly in $c \leq \beta \leq c^{\prime}$ for arbitrary $c \leq c^{\prime}$. For $q=0$ we simply write $M_{\mathcal{O}}^{\mu}(X)$. The space $M_{\mathcal{O}}^{\mu}\left(X ; \mathbb{R}^{q}\right)$ is Fréchet in a canonical way. So we can form spaces of the kind $C^{\infty}\left(\left(\overline{\mathbb{R}}_{+}\right)^{2} \times U, M_{\mathcal{O}}^{\mu}\left(X ; \mathbb{R}^{q}\right)\right)$, etc. Let $M_{\mathcal{O}}^{\mu}\left(X ;\left(\overline{\mathbb{R}}_{+}\right)^{2} \times U \times\right.$ $\left.\mathbb{R}^{q}\right)_{\text {edge }}$ denote the subspace of all $h\left(r, r^{\prime}, y, z, \eta\right) \in C^{\infty}\left(\left(\mathbb{R}_{+}\right)^{2} \times U, M_{\mathcal{O}}^{\mu}\left(X ; \mathbb{R}^{q}\right)\right)$ 
such that there is an $\widetilde{h}\left(r, r^{\prime}, y, z, \widetilde{\eta}\right) \in C^{\infty}\left(\left(\overline{\mathbb{R}}_{+}\right)^{2} \times U, M_{\mathcal{O}}^{\mu}\left(X ; \mathbb{R}_{\tilde{\eta}}\right)\right)$ for which $h\left(r, r^{\prime}, y, z, \eta\right)=\widetilde{h}\left(r, r^{\prime}, y, z, r \eta\right)$.

Theorem 1.3.1 ([29, Section 3.2.2]). For every $p\left(r, r^{\prime}, y, \varrho, \eta\right) \in L_{\mathrm{cl}}^{\mu}(X ;$ $\left.\left(\overline{\mathbb{R}}_{+}\right)^{2} \times U \times \mathbb{R}^{1+q}\right)_{\text {edge }}$ there exists an $h\left(r, r^{\prime}, y, z, \eta\right) \in M_{\mathcal{O}}^{\mu}\left(X ;\left(\overline{\mathbb{R}}_{+}\right)^{2} \times U \times\right.$ $\left.\mathbb{R}^{q}\right)_{\text {edge }}$ such that

$$
\operatorname{op}_{r}(p)(y, \eta)=\operatorname{op}_{M}^{\beta}(h)(y, \eta) \bmod C^{\infty}\left(U, L^{-\infty}\left(X^{\wedge} ; \mathbb{R}^{q}\right)\right)
$$

for each $\beta \in \mathbb{R}$.

The Mellin symbol $h$ is the result of a certain kernel cut-off procedure, cf. also [23], [25]. An inspection of the proof shows that the variables $\left(r, r^{\prime}\right)$ in the operator function $p$ remain untouched in the construction of $\widetilde{h}\left(r, r^{\prime}, y, z, \widetilde{\eta}\right)$. In particular, if $p$ vanishes for $\left|r, r^{\prime}\right|>c$ for a constant $c>0$, we can choose $\widetilde{h}$ in such a way that it also vanishes for $\left|r, r^{\prime}\right|>c$.

Remark 1.3.2. If we set

$$
p_{0}(r, y, \varrho, \eta):=\widetilde{p}(0,0, y, r \varrho, r \eta), \quad h_{0}(r, y, z, \eta):=\widetilde{h}(0,0, y, z, r \eta),
$$

where $\widetilde{h}$ is associated with $p$ in the sense of the constructions for Theorem 1.3.1, we have $\operatorname{op}_{r}\left(p_{0}\right)(y, \eta)=\operatorname{op}_{M}^{\beta}\left(h_{0}\right)(y, \eta) \bmod C^{\infty}\left(U, L^{-\infty}\left(X^{\wedge} ; \mathbb{R}^{q}\right)\right)$ for each $\beta \in$ $\mathbb{R}$.

For the following theorem we fix cut-off functions $\omega(r), \widetilde{\omega}(r)$ satisfying $\omega \widetilde{\omega}=\omega$, set $\chi:=1-\omega$ and choose any $\tilde{\chi} \in C^{\infty}\left(\overline{\mathbb{R}}_{+}\right)$vanishing near 0 such that $\chi \tilde{\chi}=\chi$.

Theorem 1.3.3 ([29], [9]). Let $p\left(r, r^{\prime}, y, \varrho, \eta\right) \in L_{\mathrm{cl}}^{\mu}\left(X ;\left(\overline{\mathbb{R}}_{+}\right)^{2} \times U \times\right.$ $\left.\mathbb{R}^{1+q}\right)_{\text {edge }}$ be of the form (1.3.2), and assume that $p$ vanishes for $\left|r, r^{\prime}\right| \geq c$ for some $c>0$. Let $h\left(r, r^{\prime}, y, z, \eta\right) \in M_{\mathcal{O}}^{\mu}\left(X ;\left(\overline{\mathbb{R}}_{+}\right)^{2} \times U \times \mathbb{R}^{q}\right)_{\text {edge }}$ be associated with $p$ in the sense of Theorem 1.3.1, and set

$$
\begin{aligned}
a_{\psi}(y, \eta):= & r^{-\mu}\left\{\omega(r[\eta]) \operatorname{op}_{M}^{\gamma-(n / 2)}(h)(y, \eta) \widetilde{\omega}(r[\eta])\right. \\
& \left.+\chi(r[\eta]) \operatorname{op}_{r}(p)(y, \eta) \widetilde{\chi}(r[\eta])\right\} .
\end{aligned}
$$

Then we have

$$
a_{\psi}(y, \eta) \in S^{\mu}\left(U \times \mathbb{R}^{q} ; E, \widetilde{E}\right)
$$

for $E=\mathcal{K}^{s, \gamma}\left(X^{\wedge}\right), \widetilde{E}=\mathcal{K}^{s-\mu, \gamma-\mu}\left(X^{\wedge}\right)$ as well as for $E=\mathcal{K}_{P}^{s, \gamma}\left(X^{\wedge}\right)$ and $\widetilde{E}=\mathcal{K}_{Q}^{s-\mu, \gamma-\mu}\left(X^{\wedge}\right)$ for every $P \in \operatorname{As}\left(X,(\gamma, \Theta)^{(\bullet)}\right)$ with some resulting $Q \in$ $\operatorname{As}\left(X,(\gamma-\mu, \Theta)^{(\bullet)}\right)$, for arbitrary $\Theta$, and all $s \in \mathbb{R}$. 
Remark 1.3.4. If $b\left(r, r^{\prime}, y, \varrho, \eta\right) \in L^{-\infty}\left(X ;\left(\mathbb{R}_{+}\right)^{2} \times U \times \mathbb{R}^{1+q}\right)$ vanishes for $\left|r, r^{\prime}\right|>c$ we have

$$
g(y, \eta):=r^{-\mu} \chi(r[\eta]) \mathrm{op}_{r}(b)(y, \eta) \widetilde{\chi}(r[\eta]) \in R_{G}^{-\infty}\left(U \times \mathbb{R}^{q}, \boldsymbol{g}\right)
$$

for $\boldsymbol{g}=(\gamma, \delta,(-\infty, 0])$ for arbitrary $\gamma, \delta \in \mathbb{R}$. This gives us an extension of Theorem 1.3.3 to arbitrary $p \in L_{\mathrm{cl}}^{\mu}\left(X ;\left(\overline{\mathbb{R}}_{+}\right)^{2} \times U \times \mathbb{R}^{1+q}\right)$ vanishing for $\left|r, r^{\prime}\right|>c$, when the Mellin symbols $h$ belonging to $b$ are defined to be zero.

Definition 1.3.5. Let $\mathcal{R}^{\mu}\left(U \times \mathbb{R}^{q}, \boldsymbol{g} ; j_{-}, j_{+}\right)$for $\boldsymbol{g}=(\gamma, \gamma-\mu, \Theta)$ defined to be the set of all operator families $\left(\begin{array}{cc}a_{\psi} & 0 \\ 0 & 0\end{array}\right)(y, \eta)+(m+g)(y, \eta)$ for arbitrary $a(y, \eta)$ of the form (1.3.3) and $(m+g)(y, \eta) \in \mathcal{R}_{M+G}^{\mu}\left(U \times \mathbb{R}^{q}, \boldsymbol{g} ; j_{-}, j_{+}\right)$. For $j_{-}=j_{+}=0$ we simply write $R^{\mu}\left(U \times \mathbb{R}^{q}, \boldsymbol{g}\right)$. The elements of $\mathcal{R}^{\mu}(U \times$ $\left.\mathbb{R}^{q}, \boldsymbol{g} ; j_{-}, j_{+}\right)$are called edge symbols with continuous asymptotics.

Analogous notation makes sense for discrete asymptotics, indicated by $\boldsymbol{g}^{\bullet}$. Here, in connection with edge symbols we will mainly discuss continuous asymptotics.

There is an evident generalisation of our $2 \times 2$-block matrix symbol classes to the case that the operators in the upper left corners are $f \times e$-matrices, pointwise acting in the sense $\mathcal{K}^{s, \gamma}\left(X^{\wedge}, \mathbb{C}^{e}\right) \rightarrow \mathcal{K}^{s-\mu, \gamma-\mu}\left(X^{\wedge}, \mathbb{C}^{f}\right)$ where

$$
\mathcal{K}^{s, \gamma}\left(X^{\wedge}, \mathbb{C}^{N}\right):=\mathcal{K}^{s, \gamma}\left(X^{\wedge}\right) \otimes \mathbb{C}^{N}
$$

We then have the symbol spaces

$$
\mathcal{R}^{\mu}\left(U \times \mathbb{R}^{q}, \boldsymbol{g} ; \boldsymbol{v}\right)
$$

and the corresponding subspaces with subscripts " $M+G$ " and " $G$ ", with weight data $\boldsymbol{g}=(\gamma, \gamma-\mu, \Theta)$ and dimension data $\boldsymbol{v}=\left(e, f ; j_{-}, j_{+}\right)$.

We also have symbol spaces (1.3.5) for $\Theta=(-\infty, 0]$ that are defined to be intersections of corresponding spaces for $\Theta=(-(k+1), 0]$ over $k \in \mathbb{N}$.

Remark 1.3.6. Write $\eta=\left(\eta^{\prime}, \eta^{\prime \prime}\right)$ for $\eta^{\prime} \in \mathbb{R}^{q^{\prime}}, \eta^{\prime \prime} \in \mathbb{R}^{q^{\prime \prime}}, q=q^{\prime}+q^{\prime \prime}$. Then $a(y, \eta) \in \mathcal{R}^{\mu}\left(U \times \mathbb{R}^{q}, \boldsymbol{g} ; \boldsymbol{v}\right)$ implies $a\left(y, \eta^{\prime}, \eta_{0}^{\prime \prime}\right) \in \mathcal{R}^{\mu}\left(U \times \mathbb{R}^{q^{\prime}}, \boldsymbol{g} ; \boldsymbol{v}\right)$ for every fixed $\eta_{0}^{\prime \prime} \in \mathbb{R}^{q^{\prime \prime}}$. To verify this property it suffices to observe that the function $\eta \rightarrow[\eta]$ may be replaced by any other strictly positive function $c(\eta)$ that is a classical symbol in $\eta$ of order zero. 


\section{$\S 1.4$. Operators on a manifold with edges}

We now turn to operators $\operatorname{Op}(a) u(y)=\iint e^{i\left(y-y^{\prime}\right) \eta} a(y, \eta) u\left(y^{\prime}\right) d y^{\prime} d \eta$ with respect to the local edge variables $y \in \Omega, \Omega \subseteq \mathbb{R}^{q}$ open, $a(y, \eta) \in \mathcal{R}^{\mu}(\Omega \times$ $\left.\mathbb{R}^{q}, \boldsymbol{g} ; \boldsymbol{v}\right)$. Concerning the dimension data we first assume $\boldsymbol{v}=\left(e, f ; j_{-}, j_{+}\right)$; later on we replace this by a tuple of vector bundles $\left(E, F ; J_{-}, J_{+}\right)$with the corresponding fibre dimensions. Moreover, if we say nothing else, we set $\boldsymbol{g}=$ $(\gamma, \gamma-\mu, \Theta)$ for $\Theta=(-(k+1), 0], k \in \mathbb{N} \cup\{\infty\}$.

The operators will be continuous in edge Sobolev spaces, based on the following general definition from [24]. Given a Hilbert space $H$ with group action $\left\{\kappa_{\lambda}\right\}_{\lambda \in \mathbb{R}_{+}}$, the ("abstract" edge-) space $\mathcal{W}^{s}\left(\mathbb{R}^{q}, H\right), s \in \mathbb{R}$, is defined to be the completion of $\mathcal{S}\left(\mathbb{R}^{q}, H\right)$ with respect to the norm $\left\{\int\left\|\langle\eta\rangle^{s} \kappa_{\langle\eta\rangle}^{-1} \hat{u}(\eta)\right\|_{H}^{2} d \eta\right\}^{1 / 2}$. Moreover, if $H=\lim _{j \in \mathbb{N}} H^{j}$ is a Fréchet space with group action, we set $\mathcal{W}^{s}\left(\mathbb{R}^{q}, H\right)=\varliminf_{j \in \mathbb{N}} \mathcal{W}^{s}\left(\mathbb{R}^{q}, H^{j}\right)$.

Similarly to the "comp"- and "loc"-versions of standard Sobolev spaces, we can define spaces $\mathcal{W}_{\text {comp }}^{s}(\Omega, H)$ and $\mathcal{W}_{\text {loc }}^{s}(\Omega, H)$ for any open set $\Omega \subseteq \mathbb{R}^{q}$. Further properties of such spaces may be found in [26], [29]. In particular, for $H$ we can insert the weighted cone Sobolev spaces $\mathcal{K}^{s, \gamma}\left(X^{\wedge}\right)$ and subspaces with asymptotics, cf. (1.1.12).

Set

$$
\begin{aligned}
\mathcal{W}_{\text {comp }}^{s, \gamma}\left(X^{\wedge} \times \Omega\right) & :=\mathcal{W}_{\text {comp }}^{s}\left(\Omega, \mathcal{K}^{s, \gamma}\left(X^{\wedge}\right)\right), \\
\mathcal{W}_{\text {loc }}^{s, \gamma}\left(X^{\wedge} \times \Omega\right) & :=\mathcal{W}_{\text {loc }}^{s}\left(\Omega, \mathcal{K}^{s, \gamma}\left(X^{\wedge}\right)\right) .
\end{aligned}
$$

Notice that "comp" and "loc" on the left hand sides only refer to the $y$ variables. Analogous notation will be used with subscript $P \in \operatorname{As}(X,(\gamma, \Theta))$, as well as for vector-valued functions, e.g., $\mathcal{W}_{\text {comp }}^{s, \gamma}\left(X^{\wedge} \times \Omega, \mathbb{C}^{N}\right):=\mathcal{W}_{\text {comp }}^{s}(\Omega$, $\left.\mathcal{K}^{s, \gamma}\left(X^{\wedge}, \mathbb{C}^{N}\right)\right), \mathcal{K}^{s, \gamma}\left(X^{\wedge}, \mathbb{C}^{N}\right):=\mathcal{K}^{s, \gamma}\left(X^{\wedge}\right) \otimes \mathbb{C}^{N}$, etc.

Moreover, for $\boldsymbol{m}:=(e, j)$ we set

$$
\begin{aligned}
\mathcal{W}_{\text {comp }}^{s, \gamma}\left(X^{\wedge} \times \Omega ; \boldsymbol{m}\right) & :=\mathcal{W}_{\text {comp }}^{s, \gamma}\left(X^{\wedge} \times \Omega, \mathbb{C}^{e}\right) \oplus H^{s-\frac{n+1}{2}}\left(\Omega, \mathbb{C}^{j}\right), \\
\mathcal{W}_{P, \text { comp }}^{s, \gamma}\left(X^{\wedge} \times \Omega ; \boldsymbol{m}\right): & =\mathcal{W}_{P, \text { comp }}^{s, \gamma}\left(X^{\wedge} \times \Omega, \mathbb{C}^{e}\right) \oplus H^{s-\frac{n+1}{2}}\left(\Omega, \mathbb{C}^{j}\right),
\end{aligned}
$$

and, analogously, with subscript "loc", for all $s \in \mathbb{R}$ and every $P \in \operatorname{As}(X,(\gamma$, $\Theta))$.

In the following theorem we set $\boldsymbol{m}=\left(e, j_{-}\right), \boldsymbol{n}=\left(f, j_{+}\right), \boldsymbol{v}=\left(e, f ; j_{-}, j_{+}\right)$.

Theorem 1.4.1. For every $a(y, \eta) \in \mathcal{R}^{\mu}\left(\Omega \times \mathbb{R}^{q}, \boldsymbol{g} ; \boldsymbol{v}\right)$ the operator $\mathrm{Op}(a): C_{0}^{\infty}\left(X^{\wedge} \times \Omega, \mathbb{C}^{e}\right) \oplus C_{0}^{\infty}\left(\Omega, \mathbb{C}^{j_{-}}\right) \rightarrow C^{\infty}\left(X^{\wedge} \times \Omega, \mathbb{C}^{f}\right) \oplus C^{\infty}\left(\Omega, \mathbb{C}^{j_{+}}\right)$ extends to continuous operators

$$
\mathrm{Op}(a): \mathcal{W}_{\text {comp }}^{s, \gamma}\left(X^{\wedge} \times \Omega ; \boldsymbol{m}\right) \rightarrow \mathcal{W}_{\mathrm{loc}}^{s-\mu, \gamma-\mu}\left(X^{\wedge} \times \Omega ; \boldsymbol{n}\right)
$$


and

$$
\mathrm{Op}(a): \mathcal{W}_{P, \text { comp }}^{s, \gamma}\left(X^{\wedge} \times \Omega ; \boldsymbol{m}\right) \rightarrow \mathcal{W}_{Q, \text { loc }}^{s-\mu, \gamma-\mu}\left(X^{\wedge} \times \Omega ; \boldsymbol{n}\right)
$$

for all $s \in \mathbb{R}$ and every $P \in \operatorname{As}(X,(\gamma, \Theta))$ with some $Q \in \operatorname{As}(X,(\gamma-\mu, \Theta))$ (only depending on a and $P$, not on $s$ ).

This result is a consequence of the fact that operator-valued symbols as defined in Section 1.1 give rise to continuous operators in abstract edge spaces, and of Theorems 1.2.2 and 1.3.3.

Let Vect $(\cdot)$ denote the set of all smooth complex vector bundles on the space in the brackets. In particular, for a (stretched) manifold $\mathbb{W}$ with edges we shall represent bundles locally on (stretched) wedges $\overline{\mathbb{R}}_{+} \times X \times \Omega$ with respect to the singular charts in the above-mentioned form, where transition maps will assumed to be homogeneous of order 1 with respect to homotheties in the axial variable $r \in \overline{\mathbb{R}}_{+}$.

To avoid too complicated notation we assume $\partial \mathbb{W}$ to be a trivial $X$-bundle, such that a neighbourhood of $\partial \mathbb{W}$ corresponds to $\overline{\mathbb{R}}_{+} \times X \times Y$ with $Y$ being the edge, and the Riemannian metric on $\mathbb{W}$ near $\partial \mathbb{W}$ is supposed to be the product metric of the metric from the Lebesgue measure on $\overline{\mathbb{R}}_{+}$and Riemannian metrics on $X$ and $Y$. We only consider vector bundles $E \in \operatorname{Vect}(\mathbb{W})$ such that the restriction of $E$ to this neighbourhood is the pull-back of some bundle $E_{0}$ on $X$ with respect to the projection $\overline{\mathbb{R}}_{+} \times X \times Y \rightarrow X$. Let $E^{\prime}$ denote the pull-back of $E_{0}$ with respect to the projection $X \times Y \rightarrow X$.

The occurring complex vector bundles are assumed to be equipped with Hermitian metrics. Hermitian metrics in bundles on $X^{\wedge}$ are assumed to be homogeneous of degree 0 with respect to homotheties in the axial variables $r \in \mathbb{R}_{+}$.

Using invariance (recall that our atlas on $\mathbb{W}$ is fixed and specified) we can define global spaces

$$
\mathcal{W}_{\text {comp }}^{s, \gamma}(\mathbb{W}, E), \quad \mathcal{W}_{\text {loc }}^{s, \gamma}(\mathbb{W}, E)
$$

of distributional sections, where "comp" and "loc" (similarly to the local meaning of notation) does not exclude supports up to $\partial \mathbb{W}$ (though we might write int $\mathbb{W}$ in the spaces, because Sobolev spaces are used to be defined in int $\mathbb{W}=$ $\mathbb{W} \backslash \partial \mathbb{W}$; nevertheless, for simplicity, we write $\mathbb{W}$ in the spaces and hope that this will not lead to confusion).

In a similar sense we get subspaces $\mathcal{W}_{P, \text { comp }}^{s, \gamma}(\mathbb{W}, E)$ and $\mathcal{W}_{P, \text { loc }}^{s, \gamma}(\mathbb{W}, E)$ for asymptotic types $P$; the coefficients of asymptotics then belong to $C^{\infty}\left(X, E_{0}\right)$. 
Writing $P \in \operatorname{As}(X, \boldsymbol{g})$ (or $P \in \operatorname{As}\left(X, g^{\bullet}\right)$ ) we mean in this case the obvious generalisation to the bundle case. Note that the "comp"- and "loc"-spaces (with or without asymptotics) are locally outside a neighbourhood of $\partial \mathbb{W}$ in the standard Sobolev spaces of smoothness $s$ (with the corresponding specified support in the "comp"-case).

Set $\boldsymbol{m}:=(E, J)$ for $E \in \operatorname{Vect}(\mathbb{W}), J \in \operatorname{Vect}(Y)$ and

$$
\begin{aligned}
\mathcal{W}_{\text {comp }}^{s, \gamma}(\mathbb{W} ; \boldsymbol{m}) & :=\mathcal{W}_{\text {comp }}^{s, \gamma}(\mathbb{W}, E) \oplus H_{\text {comp }}^{s-\frac{n+1}{2}}(Y, J), \\
\mathcal{W}_{P, \text { comp }}^{s, \gamma}(\mathbb{W} ; \boldsymbol{m}) & :=\mathcal{W}_{P, \text { comp }}^{s, \gamma}(\mathbb{W}, E) \oplus H_{\text {comp }}^{s-\frac{n+1}{2}}(Y, J),
\end{aligned}
$$

for $P \in \operatorname{As}(X, \boldsymbol{g})$ (or $P \in \operatorname{As}\left(X, g^{\bullet}\right)$ ). In an analogous manner we define the corresponding spaces with subscript "loc".

If $\mathbb{W}$ is compact, we may omit "comp" or "loc" and simply write

$$
\mathcal{W}^{s, \gamma}(\mathbb{W} ; \boldsymbol{m}) \quad \text { and } \quad \mathcal{W}_{P}^{s, \gamma}(\mathbb{W} ; \boldsymbol{m}),
$$

respectively.

In the spaces $\mathcal{W}_{\text {loc }}^{0,0}(\mathbb{W}, E), E \in \operatorname{Vect}(\mathbb{W})$, and $H_{\text {loc }}^{0}(Y, J), J \in \operatorname{Vect}(Y)$, we fix sesquilinear pairings, based on the chosen Riemannian metrics and Hermitian metrics in the bundles, that induce scalar products in subspaces $\mathcal{W}^{0,0}(\mathbb{W}, E)_{K}:=\left\{u \in \mathcal{W}_{\text {loc }}^{0,0}(\mathbb{W}, E): \operatorname{supp} u \subseteq K\right\}$ and $H^{0}(Y, J)_{K^{\prime}}:=\{v \in$ $\left.H_{\mathrm{loc}}^{0}(Y, J): \operatorname{supp} v \subseteq K^{\prime}\right\}$ for arbitrary compact subsets $K \subseteq \mathbb{W}$ and $K^{\prime} \subseteq Y$, respectively. This gives us sesquilinear pairings $\mathcal{W}_{\text {comp }}^{s, \gamma}(\mathbb{W}, E) \times \mathcal{W}_{\text {loc }}^{-s,-\gamma}(\mathbb{W}, E)$ $\rightarrow \mathbb{C}$ and $H_{\text {comp }}^{s}(Y, J) \times H_{\text {loc }}^{-s}(Y, J) \rightarrow \mathbb{C}$ for all $s, \gamma \in \mathbb{R}$, or, in more concise description,

$$
\mathcal{W}_{\text {comp }}^{s, \gamma}(\mathbb{W} ; \boldsymbol{m}) \times \mathcal{W}_{\text {loc }}^{(-s)^{*},-\gamma}(\mathbb{W} ; \boldsymbol{m}) \rightarrow \mathbb{C}
$$

for $\boldsymbol{m}=(E, J)$ and $\mathcal{W}_{\mathrm{loc}}^{(-s)^{*},-\gamma}(\mathbb{W} ; \boldsymbol{m}):=\mathcal{W}_{\mathrm{loc}}^{-s,-\gamma}(\mathbb{W}, E) \oplus H_{\mathrm{loc}}^{-s+(n+1) / 2}(Y, J)$.

An operator $\mathcal{C}: \mathcal{W}_{\text {comp }}^{s, \gamma}(\mathbb{W} ; \boldsymbol{m}) \rightarrow \mathcal{W}^{\infty, \gamma-\mu}(\mathbb{W} ; \boldsymbol{n})$ for $\boldsymbol{m}:=\left(E, J_{-}\right), \boldsymbol{n}:=$ $\left(F, J_{+}\right), E, F \in \operatorname{Vect}(\mathbb{W}), J_{ \pm} \in \operatorname{Vect}(Y)$, is said to belong to $\mathcal{Y}^{-\infty}(\mathbb{W}, \boldsymbol{g} ; \boldsymbol{v})$ for $\boldsymbol{g}=(\gamma, \delta, \Theta), \Theta=(\vartheta, 0], \boldsymbol{v}=\left(E, F ; J_{-}, J_{+}\right), E, F \in \operatorname{Vect}(\mathbb{W}), J_{ \pm} \in \operatorname{Vect}(Y)$, if $\mathcal{C}$ induces continuous operators

$$
\begin{gathered}
\mathcal{C}: \mathcal{W}_{\text {comp }}^{s, \gamma}(\mathbb{W} ; \boldsymbol{m}) \rightarrow \mathcal{W}_{P, \text { loc }}^{\infty, \delta}(\mathbb{W} ; \boldsymbol{n}), \\
\mathcal{C}^{*}: \mathcal{W}_{\text {comp }}^{(-s)^{*},-\delta}(\mathbb{W} ; \boldsymbol{n}) \rightarrow \mathcal{W}_{Q,- \text { loc }}^{\infty,-\gamma}(\mathbb{W} ; \boldsymbol{m})
\end{gathered}
$$

for all $s \in \mathbb{R}$ and asymptotic types $P \in \operatorname{As}(X,(\delta, \Theta)), Q \in \operatorname{As}(X,(-\gamma, \Theta))$, depending on the operator $\mathcal{C}$; here, $\mathcal{C}^{*}$ is the formal adjoint of $\mathcal{C}$ with respect to the pairing (1.4.1). 
Remark 1.4.2. The space $\mathcal{Y}^{-\infty}(\mathbb{W}, \boldsymbol{g} ; \boldsymbol{v})_{P, Q}$ of all elements $\mathcal{Y}^{-\infty}(\mathbb{W}, \boldsymbol{g} ; \boldsymbol{v})$ with given $P$ and $Q$ is Fréchet in a canonical way.

We then set

$$
\mathcal{Y}^{-\infty}\left(\mathbb{W}, \boldsymbol{g} ; \boldsymbol{v} ; \mathbb{R}^{l}\right)_{P, Q}:=\mathcal{S}\left(\mathbb{R}^{l}, \mathcal{Y}^{-\infty}(\mathbb{W}, \boldsymbol{g} ; \boldsymbol{v})_{P, Q}\right)
$$

and define $\mathcal{Y}^{-\infty}\left(\mathbb{W}, \boldsymbol{g} ; \boldsymbol{v} ; \mathbb{R}^{l}\right)$ to be the union of the spaces (1.4.2), where $P, Q$ run through all asymptotic types associated with the given weight data.

In particular, we have the subspace

$$
\mathcal{Y}^{-\infty}\left(\mathbb{W}, \boldsymbol{g} ; \boldsymbol{v} ; \mathbb{R}^{l}\right)_{\mathcal{O}}
$$

of all smoothing operators, where the asymptotic types $P$ and $Q$ are the trivial ones in $\operatorname{As}(X,(\delta, \Theta))$ and $\operatorname{As}(X,(-\gamma, \Theta))$, respectively.

To define edge pseudo-differential operators with parameters we start from the space of operator-valued symbols $\mathcal{R}^{\mu}\left(\Omega \times \mathbb{R}^{q+l}, \boldsymbol{g} ; \boldsymbol{w}\right)$ for open $\Omega \subseteq \mathbb{R}^{q}$, $\boldsymbol{g}=(\gamma, \gamma-\mu, \Theta), \boldsymbol{w}:=\left(e, f ; j_{-}, j_{+}\right)$. Globally, we consider vector bundles $\boldsymbol{v}=\left(E, F ; J_{-}, J_{+}\right)$with the numbers in $\boldsymbol{w}$ as fibre dimensions. In Section 1.1 we have introduced singular charts $\alpha: V \rightarrow X^{\Delta} \times \Omega$ on $W$. These induce charts $\alpha^{\prime}: V \cap Y \rightarrow \Omega$ on $Y$ and stretched charts $\bar{\alpha}: \mathbb{V} \rightarrow \overline{\mathbb{R}}_{+} \times X \times \Omega$ on $\mathbb{W}$. In addition, we have interior charts $\iota: U \rightarrow G$, for coordinate neighbourhoods $U \subset \operatorname{int} \mathbb{W}, G \subseteq \mathbb{R}^{n+1+q}$ open.

On int $\mathbb{W}$ we have the space $L_{\mathrm{cl}}^{\mu}\left(\mathrm{int} \mathbb{W} ; E, F ; \mathbb{R}^{l}\right)$ of classical parameterdependent pseudo-differential operators, acting between spaces of distributional sections in $E$ and $F$ (classical means that local amplitude functions are classical symbols in covariables including parameters). Similarly, we have the space $L_{\mathrm{cl}}^{\mu}\left(Y ; J_{-}, J_{+} ; \mathbb{R}^{l}\right)$.

Let $\chi, \tilde{\chi} \in C^{\infty}(\mathbb{W})$ be functions that equal 0 for $\operatorname{dist}(\widetilde{x}, \partial \mathbb{W})<\varepsilon_{0}$ and 1 for $\operatorname{dist}(\widetilde{x}, \partial \mathbb{W})>\varepsilon_{1}, \widetilde{x} \in \mathbb{W}$, for sufficiently small $0<\varepsilon_{0}<\varepsilon_{1}<1$.

Then $\mathcal{Y}^{\mu}\left(\mathbb{W}, \boldsymbol{g} ; \boldsymbol{v} ; \mathbb{R}^{l}\right)$ is defined to be the set of all block matrix families

$$
\mathcal{A}(\lambda)=\left(\begin{array}{cc}
\chi A_{\text {int }}(\lambda) \tilde{\chi} & 0 \\
0 & 0
\end{array}\right)+\mathcal{B}(\lambda)+\mathcal{C}(\lambda)
$$

for arbitrary $\mathcal{C}(\lambda) \in \mathcal{Y}^{-\infty}\left(\mathbb{W}, \boldsymbol{g} ; \boldsymbol{v} ; \mathbb{R}^{l}\right), A_{\text {int }}(\lambda) \in L_{\mathrm{cl}}^{\mu}\left(\operatorname{int} \mathbb{W} ; E, F ; \mathbb{R}^{l}\right)$, and $\mathcal{B}(\lambda)$ being a locally finite sum of operators of the form

$$
\left(\bar{\alpha}_{j}^{-1}\right)_{*} \delta_{j} \mathrm{Op}_{y}\left(a_{j}\right)(\lambda) \widetilde{\delta}_{j}, \quad j \in \mathbb{N}
$$

with respect to charts $\alpha_{j}: V_{j} \rightarrow X^{\Delta} \times \Omega_{j}$, for arbitrary $a_{j}(y, \eta, \lambda) \in \mathcal{R}^{\mu}(\Omega \times$ $\left.\mathbb{R}_{\eta, \lambda}^{q+l}, \boldsymbol{g} ; \boldsymbol{w}\right), \delta_{j}, \widetilde{\delta}_{j} \in C_{0}^{\infty}(\Omega)$ with $\delta_{j} \widetilde{\delta}_{j}=\delta_{j}$, where $\left(\left(\alpha_{j}^{\prime}\right)^{*} \delta_{j}\right)_{j \in \mathbb{N}}$ is a partition of 
unity on $Y$. The interpretation of (1.4.5) is that the local pseudo-differential operator $\delta_{j} \operatorname{Op}_{y}\left(a_{j}\right)(\lambda) \widetilde{\delta}_{j}$ with operator-valued symbol $a_{j}$ is operator-pulled back to a stretched neighbourhood $\mathbb{V}_{j}$ with respect to the corresponding singular chart $\bar{\alpha}_{j}: \mathbb{V}_{j} \rightarrow \overline{\mathbb{R}}_{+} \times X \times \Omega_{j}$ and isomorphisms $\left.E\right|_{\mathbb{V}_{j}} \rightarrow\left(\overline{\mathbb{R}}_{+} \times X \times \Omega_{j}\right) \times \mathbb{C}^{e}$, $\left.J_{-}\right|_{V_{j} \cap Y} \rightarrow \Omega_{j} \times \mathbb{C}^{j-}$, etc. Here, we assume that the system $\left(\mathbb{V}_{j}\right)_{j \in \mathbb{N}}$ covers a neighbourhood of $\partial \mathbb{W}$ in $\mathbb{W}$. The definition is correct, since we have invariance of operators $\delta_{j} \mathrm{Op}\left(a_{j}\right)(\lambda) \widetilde{\delta}_{j}$ under transition maps, modulo elements of order $-\infty$ and because upper left corners of operators of the form $\mathcal{B}(\lambda)$ belong to $L_{\text {cl }}^{\mu}\left(\right.$ int $\left.\mathbb{W} ; E, F ; \mathbb{R}^{l}\right)$.

In particular, $\mathcal{Y}_{M+G}^{\mu}\left(\mathbb{W}, \boldsymbol{g} ; \boldsymbol{v} ; \mathbb{R}^{l}\right)$ denotes the subset of all elements $\mathcal{A}(\lambda)$ in $\mathcal{Y}_{M+G}^{\mu}\left(\mathbb{W}, \boldsymbol{g} ; \boldsymbol{v} ; \mathbb{R}^{l}\right)$ where the local amplitude functions $a_{j}$ belong to $\mathcal{R}_{M+G}^{\mu}(\Omega \times$ $\left.\mathbb{R}^{q+l}, \boldsymbol{g} ; \boldsymbol{w}\right)$ for all $j$ and $A_{\text {int }}(\lambda) \in L^{-\infty}\left(\right.$ int $\left.\mathbb{W} ; E, F ; \mathbb{R}^{l}\right)$. Analogously we define $\mathcal{Y}_{G}^{\mu}\left(\mathbb{W}, \boldsymbol{g} ; \boldsymbol{v} ; \mathbb{R}^{l}\right)$ by requiring $a_{j}$ to be a Green amplitude function for all $j$. The latter operator families are called parameter-dependent Green operators on $\mathbb{W}$.

As a consequence of Theorem 1.4.1 we have the following assertion:

Theorem 1.4.3. Let $\boldsymbol{g}=(\gamma, \gamma-\mu, \Theta), \boldsymbol{v}=\left(E, F ; J_{-}, J_{+}\right)$, and $\boldsymbol{m}=$ $\left(E, J_{-}\right), \boldsymbol{n}=\left(F, J_{+}\right)$. An element $\mathcal{A} \in \mathcal{Y}^{\mu}\left(\mathbb{W}, \boldsymbol{g} ; \boldsymbol{v} ; \mathbb{R}_{\lambda}^{l}\right)$ induces families of continuous operators

$$
\mathcal{A}(\lambda): \mathcal{W}_{\text {comp }}^{s, \gamma}(\mathbb{W} ; \boldsymbol{m}) \rightarrow \mathcal{W}_{\mathrm{loc}}^{s-\mu, \gamma-\mu}(\mathbb{W} ; \boldsymbol{n})
$$

and

$$
\mathcal{A}(\lambda): \mathcal{W}_{P, \text { comp }}^{s, \gamma}(\mathbb{W} ; \boldsymbol{m}) \rightarrow \mathcal{W}_{Q, \text { loc }}^{s-\mu, \gamma-\mu}(\mathbb{W} ; \boldsymbol{n})
$$

for all $s \in \mathbb{R}, \lambda \in \mathbb{R}^{l}$, and for every $P \in \operatorname{As}(X,(\gamma, \Theta))$ with some $Q \in$ $\operatorname{As}(X,(\gamma-\mu, \Theta))$.

By definition, the elements of $\mathcal{Y}^{\mu}\left(\mathbb{W}, \boldsymbol{g} ; \boldsymbol{v} ; \mathbb{R}^{l}\right)$ are $2 \times 2$-block matrices, where the upper left corners refer to the bundles $(E, F)$ contained in $\boldsymbol{v}=$ $\left(E, F ; J_{-}, J_{+}\right)$. Let $Y^{\mu}\left(\mathbb{W}, \boldsymbol{g} ; E, F ; \mathbb{R}^{l}\right)$ denote the space of upper left corners. Analogously, write

$$
Y_{M+G}^{\mu}\left(\mathbb{W}, \boldsymbol{g} ; E, F ; \mathbb{R}^{l}\right), \quad Y_{G}^{\mu}\left(\mathbb{W}, \boldsymbol{g} ; E, F ; \mathbb{R}^{l}\right)
$$

for the spaces of upper left corners of elements in the corresponding $2 \times 2$-block matrix spaces with subscripts $M+G$ and $G$, respectively.

The space $\mathcal{Y}_{G}^{\mu}\left(\mathbb{W}, \boldsymbol{g} ; \boldsymbol{v} ; \mathbb{R}^{l}\right)$ can be written as the union of Fréchet subspaces

$$
\mathcal{Y}_{G}^{\mu}\left(\mathbb{W}, \boldsymbol{g} ; \boldsymbol{v} ; \mathbb{R}^{l}\right)_{P, Q}
$$


over $P \in \operatorname{As}(X,(\gamma-\mu, \Theta))$ and $Q \in \operatorname{As}(X,(-\gamma, \Theta))$, where elements $\mathcal{G}$ in (1.4.9) are defined to be sums $\mathcal{G}_{\text {loc }}+\mathcal{C}$, with $\mathcal{G}_{\text {loc }}$ being given in terms of local amplitude functions in $\mathcal{R}_{G}^{\mu}\left(\Omega \times \mathbb{R}^{q+l}, \boldsymbol{g} ; \boldsymbol{w}\right)_{P, Q}$, cf. formula (1.1.18), and $\mathcal{C}$ belonging to $\mathcal{Y}^{-\infty}\left(\mathbb{W}, \boldsymbol{g} ; \boldsymbol{v} ; \mathbb{R}^{l}\right)_{P, Q}$, cf. $(1.4 .2)$.

Moreover, let us consider tuples of asymptotic types

$$
D_{1}:=\left(R_{0}, R_{1} ; P, Q\right) \quad \text { and } \quad D_{2}:=\left(R_{0}, R_{2} ; P, Q\right)
$$

in the sense of notation used in the decomposition (1.2.13) where $\left(R_{1}, R_{2} ; P, Q\right)$ are compatible. Then, similarly to (1.2.13), every $M+G \in Y_{M+G}^{\mu}(\mathbb{W}, \boldsymbol{g} ; E, F$; $\left.\mathbb{R}^{l}\right)$ is contained in a sum of vector spaces

$$
Y_{M+G}^{\mu}\left(\mathbb{W}, \boldsymbol{g} ; E, F ; \mathbb{R}^{l}\right)_{1}+Y_{M+G}^{\mu}\left(\mathbb{W}, \boldsymbol{g} ; E, F ; \mathbb{R}^{l}\right)_{2}
$$

for a suitable choice of asymptotic types $\left(R_{0}, R_{1}, R_{2} ; P, Q\right)$ with compatible $\left(R_{1}, R_{2} ; P, Q\right)$. Here, $Y_{M+G}^{\mu}\left(\mathbb{W}, \boldsymbol{g} ; E, F ; \mathbb{R}^{l}\right)_{i}$ is defined to be the set of all operators $M+G$ such that $G \in Y_{G}^{\mu}\left(\mathbb{W}, \boldsymbol{g} ; E, F ; \mathbb{R}^{l}\right)_{P, Q}$, and $M \in Y_{M+G}^{\mu}(\mathbb{W}, \boldsymbol{g} ; E$, $F ; \mathbb{R}^{l}$ ) is a locally finite sum of expressions (1.4.5) with $a_{j}$ belonging to $R_{M+G}^{\mu}\left(\Omega \times \mathbb{R}^{q+l}, \boldsymbol{g} ; e, f\right)_{i}:=R_{M+G}^{\mu}\left(\Omega \times \mathbb{R}^{q+l}, \boldsymbol{g}\right)_{i} \otimes \mathbb{C}^{f} \otimes \mathbb{C}^{e}$. The spaces

$$
Y_{M+G}^{\mu}\left(\mathbb{W}, \boldsymbol{g} ; E, F ; \mathbb{R}^{l}\right)_{i}
$$

are Fréchet in a canonical way, and we endow the space (1.4.11) with the Fréchet topology of the corresponding non-direct sum.

Let $\mathcal{Y}_{M+G}^{\mu}\left(\mathbb{W}, \boldsymbol{g} ; \boldsymbol{v} ; \mathbb{R}^{l}\right)_{i}$ denote the set of all $\mathcal{A} \in \mathcal{Y}_{M+G}^{\mu}\left(\mathbb{W}, \boldsymbol{g} ; \boldsymbol{v} ; \mathbb{R}^{l}\right), \mathcal{A}=$ $\left(\mathcal{A}_{j k}\right)_{j, k=1,2}$, where the upper left corner $\mathcal{A}_{11}$ belongs to $Y_{M+G}^{\mu}\left(\mathbb{W}, \boldsymbol{g} ; E, F ; \mathbb{R}^{l}\right)_{i}$ and $\left(\begin{array}{cc}0 & \mathcal{A}_{12} \\ \mathcal{A}_{21} & \mathcal{A}_{22}\end{array}\right)$ to $\mathcal{Y}_{G}^{\mu}\left(\mathbb{W}, \boldsymbol{g} ; \boldsymbol{v} ; \mathbb{R}^{l}\right)_{P, Q}$.

Summing up, we have the following structure:

Proposition 1.4.4. The space $\mathcal{Y}_{M+G}^{\mu}\left(\mathbb{W}, \boldsymbol{g} ; \boldsymbol{v} ; \mathbb{R}^{l}\right)$ is the union of nondirect Fréchet sums

$$
\mathcal{Y}_{M+G}^{\mu}\left(\mathbb{W}, \boldsymbol{g} ; \boldsymbol{v} ; \mathbb{R}^{l}\right)_{1}+\mathcal{Y}_{M+G}^{\mu}\left(\mathbb{W}, \boldsymbol{g} ; \boldsymbol{v} ; \mathbb{R}^{l}\right)_{2}
$$

for Fréchet subspaces $\mathcal{Y}_{M+G}^{\mu}\left(\mathbb{W}, \boldsymbol{g} ; \boldsymbol{v} ; \mathbb{R}^{l}\right)_{i} \subset \mathcal{Y}_{M+G}^{\mu}\left(\mathbb{W}, \boldsymbol{g} ; \boldsymbol{v} ; \mathbb{R}^{l}\right), i=1,2 ;$ the union is taken over all $\left(D_{1}, D_{2}\right)$ for tuples of asymptotic types (1.4.10) that are compatible in the above-mentioned sense.

Let $\mathbf{A s}_{M+G}(X, \boldsymbol{g} ; \boldsymbol{v})$ defined to be the set of all tuples $S:=\left(R_{0}, R_{1}, R_{2} ; P\right.$, $Q)$ satisfying the described compatibility, cf. similar notation of Section 1.2; here, we indicate the involved bundles $\boldsymbol{v}$ to specify the various coefficient spaces 
in the asymptotics that refer to the involved bundles. For (1.4.13) we then also write

$$
\mathcal{Y}_{M+G}^{\mu}\left(\mathbb{W}, \boldsymbol{g} ; \boldsymbol{v} ; \mathbb{R}^{l}\right)_{S}, \quad S \in \mathbf{A s}_{M+G}(X, \boldsymbol{g} ; \boldsymbol{v}) .
$$

We now define the subspace

$$
\mathcal{Y}^{\mu}\left(\mathbb{W}, \boldsymbol{g} ; \boldsymbol{v} ; \mathbb{R}^{l}\right)_{\mathcal{O}}
$$

of $\mathcal{Y}^{\mu}\left(\mathbb{W}, \boldsymbol{g} ; \boldsymbol{v} ; \mathbb{R}^{l}\right)$ to be the set of all $\mathcal{A}$ for which $\mathcal{C} \in \mathcal{Y}^{-\infty}\left(\mathbb{W}, \boldsymbol{g} ; \boldsymbol{w} ; \mathbb{R}^{l}\right)_{\mathcal{O}}$ in the representation (1.4.4), cf. formula (1.4.3), and $a_{j}(y, \eta, \lambda) \in \mathcal{R}^{\mu}(\Omega \times$ $\left.\mathbb{R}^{q+l}, \boldsymbol{g} ; \boldsymbol{w}\right)_{\mathcal{O}}$ for all $j$, cf. formula (1.4.5). Here, $\mathcal{R}^{\mu}\left(\Omega \times \mathbb{R}^{q+l} ; \boldsymbol{g} ; \boldsymbol{w}\right)_{\mathcal{O}}$ for $\boldsymbol{w}=\left(e, f ; j_{-}, j_{+}\right)$denotes the subspace of all elements $a(y, \eta, \lambda) \in \mathcal{R}^{\mu}(\Omega \times$ $\left.\mathbb{R}^{q+l}, \boldsymbol{g} ; \boldsymbol{w}\right), a=\left(a_{i j}\right)_{i, j=1,2}$, such that in the representation $a=\left(\begin{array}{cc}a_{\psi}+m & 0 \\ 0 & 0\end{array}\right)+$ $g$ (cf. analogously, Definition 1.3.5 for $e=f=1$ ) we have $g \in \mathcal{R}_{G}^{\mu}(\Omega \times$ $\left.\mathbb{R}^{q+l}, \boldsymbol{g} ; \boldsymbol{w}\right)_{\mathcal{O}}$, cf. formula $(1.1 .17)$, and where $\sigma_{M}^{(j)}(m)$ takes its values in $f \times e^{-}$ matrices with entries in $C^{\infty}\left(\Omega, M_{\mathcal{O}}^{-\infty}(X)\right) \otimes \mathcal{P}^{j}(\eta), 0 \leq j \leq k$, cf. formula (1.2.9).

The space $\mathcal{Y}^{\mu}\left(\mathbb{W}, \boldsymbol{g} ; \boldsymbol{v} ; \mathbb{R}^{l}\right)_{\mathcal{O}}$ is Fréchet in a natural way. By definition, every $\mathcal{A} \in \mathcal{Y}^{\mu}\left(\mathbb{W}, \boldsymbol{g} ; \boldsymbol{v} ; \mathbb{R}^{l}\right)$ has the form

$$
\mathcal{A}=\mathcal{A}_{\mathcal{O}}+\mathcal{M}+\mathcal{G}
$$

for certain $\mathcal{A}_{\mathcal{O}} \in \mathcal{Y}^{\mu}\left(\mathbb{W}, \boldsymbol{g} ; \boldsymbol{v} ; \mathbb{R}^{l}\right)_{\mathcal{O}}$ and $\mathcal{M}+\mathcal{G} \in \mathcal{Y}_{M+G}^{\mu}\left(\mathbb{W}, \boldsymbol{g} ; \boldsymbol{v} ; \mathbb{R}^{l}\right)$. Clearly, the decomposition (1.4.16) is not unique. Applying Proposition 1.4.4 we get the following remark.

Remark 1.4.5. Every $\mathcal{A} \in \mathcal{Y}^{\mu}\left(\mathbb{W}, \boldsymbol{g} ; \boldsymbol{v} ; \mathbb{R}^{l}\right)$ belongs to a Fréchet subspace, namely a non-direct sum of the form

$$
\mathcal{Y}^{\mu}\left(\mathbb{W}, \boldsymbol{g} ; \boldsymbol{v} ; \mathbb{R}^{l}\right)_{S}:=\mathcal{Y}^{\mu}\left(\mathbb{W}, \boldsymbol{g} ; \boldsymbol{v} ; \mathbb{R}^{l}\right)_{\mathcal{O}}+\mathcal{Y}_{M+G}^{\mu}\left(\mathbb{W}, \boldsymbol{g} ; \boldsymbol{v} ; \mathbb{R}^{l}\right)_{S}
$$

Without loss of generality we may (and will) assume that the involved asymptotic types $P, Q$ for Green operators satisfy the shadow condition.

We now introduce the principal symbols of operators $\mathcal{A}$ in $\mathcal{Y}^{\mu}\left(\mathbb{W}, \boldsymbol{g} ; \boldsymbol{v} ; \mathbb{R}^{l}\right)$. First, $A_{11}:=$ u. l. c. $\mathcal{A}$ belongs to $L_{\mathrm{cl}}^{\mu}\left(\operatorname{int} \mathbb{W} ; E, F ; \mathbb{R}^{l}\right)$. Thus there is a parameter-dependent homogeneous principal symbol of order $\mu$

$$
\sigma_{\psi}(\mathcal{A})(\widetilde{x}, \widetilde{\xi}, \lambda):=\sigma_{\psi}\left(A_{11}\right)(\widetilde{x}, \widetilde{\xi}, \lambda): \pi_{\mathrm{int} \mathbb{W}}^{*} E \rightarrow \pi_{\mathrm{int} \mathbb{W}}^{*} F,
$$

where $\pi_{\text {int } \mathbb{W}}: T^{*}(\operatorname{int} \mathbb{W}) \times \mathbb{R}^{l} \backslash 0 \rightarrow \operatorname{int} \mathbb{W}$ is the canonical projection; $(\widetilde{x}, \widetilde{\xi})$ denotes points in $T^{*}(\operatorname{int} \mathbb{W})$, and 0 stands for $(\widetilde{\xi}, \lambda)=0$. Locally near $\partial \mathbb{W}$ 
we have a splitting of variables $\widetilde{x}=(r, x, y)$ and covariables $\widetilde{\xi}=(\varrho, \xi, \eta)$, and (1.4.18) is edge-degenerate in these coordinates. Moreover,

$$
\sigma_{\psi, \mathbf{f}}(\mathcal{A})(r, x, y, \varrho, \xi, \eta, \lambda):=r^{\mu} \sigma_{\psi}(\mathcal{A})\left(r, x, y, r^{-1} \varrho, \xi, r^{-1} \eta, r^{-1} \lambda\right)
$$

is smooth up to $r=0$. We call $\sigma_{\psi, \mathbf{f}}(\mathcal{A})$ the Fuchs type symbol derived from (1.4.18); it has an invariant meaning as a homomorphism of liftings of $E$ and $F$ to a realisation of $T^{*}($ int $\mathbb{W}) \times \mathbb{R}^{l} \backslash 0$ that is connected with a special cocycle referring to the edge degeneracy of symbols. To have a notation this realisation is said to be the compressed variant of $T^{*}(\operatorname{int} \mathbb{W}) \times \mathbb{R}^{l}$, denoted $\left(T^{*} \mathbb{W} \times \mathbb{R}^{l}\right)_{\mathbf{f}}$ (roughly, covectors in $\left(T^{*} \mathbb{W} \times \mathbb{R}^{l}\right)_{\mathbf{f}}$ near $\partial \mathbb{W}$ are represented by $(\widetilde{\varrho}, \xi, \widetilde{\eta}, \widetilde{\lambda})$, where $(\varrho, \xi, \eta, \lambda)=\left(r^{-1} \widetilde{\varrho}, \xi, r^{-1} \widetilde{\eta}, r^{-1} \widetilde{\lambda}\right)$ are the "usual" covariables $)$. If $\pi_{\mathbb{W}, \mathbf{f}}$ : $\left(T^{*} \mathbb{W} \times \mathbb{R}^{l}\right)_{\mathbf{f}} \backslash 0 \rightarrow \mathbb{W}$ is the canonical projection, we then have

$$
\sigma_{\psi, \mathbf{f}}(\mathcal{A}):=\sigma_{\psi, \mathbf{f}}\left(A_{11}\right): \pi_{\mathbb{W}, \mathbf{f}}^{*} E \rightarrow \pi_{\mathbb{W}, \mathbf{f}}^{*} F
$$

This notation includes the smoothness of the homomorphism up to the boundary $\partial \mathbb{W}$.

Next we pass to the homogeneous edge symbol of order $\mu$

$$
\sigma_{\wedge}(\mathcal{A})(y, \eta, \lambda): \pi_{Y}^{*}\left(\begin{array}{c}
\mathcal{K}^{s, \gamma}\left(X^{\wedge}\right) \otimes E^{\prime} \\
\oplus \\
J_{-}
\end{array}\right) \rightarrow \pi_{Y}^{*}\left(\begin{array}{c}
\mathcal{K}^{s-\mu, \gamma-\mu}\left(X^{\wedge}\right) \otimes F^{\prime} \\
\oplus \\
J_{+}
\end{array}\right)
$$

Homogeneity of order $\mu$ means in this case that the relation

$$
\sigma_{\wedge}(\mathcal{A})(y, \delta \eta, \delta \lambda)=\delta^{\mu} \widetilde{\boldsymbol{\kappa}}_{\delta} \sigma_{\wedge}(\mathcal{A})(y, \eta, \lambda) \boldsymbol{\kappa}_{\delta}^{-1}
$$

holds for all $\delta \in \mathbb{R}_{+}$. Here,

$$
\boldsymbol{\kappa}_{\delta}:=\operatorname{diag}\left(\kappa_{\delta}, \delta^{(n+1) / 2} \operatorname{id}_{\pi_{Y}^{*} J_{-}}\right), \quad \widetilde{\boldsymbol{\kappa}}_{\delta}:=\operatorname{diag}\left(\kappa_{\delta}, \delta^{\frac{n+1}{2}} \operatorname{id}_{\pi_{Y}^{*} J_{+}}\right)
$$

(recall that $\left.\left(\kappa_{\delta} u\right)(r, x)=\delta^{(n+1) / 2} u(\delta r, x)\right)$. The upper left corner $\sigma_{\wedge}\left(A_{11}\right)$ of (1.4.21) is locally given by the expression

$$
\begin{aligned}
\sigma_{\wedge}\left(A_{11}\right)(y, \eta, \lambda)= & r^{-\mu}\left\{\omega(r|\eta, \lambda|) \operatorname{op}_{M}^{\gamma-\frac{n}{2}}\left(h_{0}\right)(y, \eta, \lambda) \widetilde{\omega}(r|\eta, \lambda|)\right. \\
& \left.+\chi(r|\eta, \lambda|) \operatorname{op}_{r}\left(p_{0}\right)(y, \eta, \lambda) \widetilde{\chi}(r|\eta, \lambda|)\right\} \\
& +\sum_{j=0}^{k} \sum_{|\alpha|=j} \sigma_{\wedge}\left(m_{j \alpha}\right)(y, \eta, \lambda)+\sigma_{\wedge}\left(g_{11}\right)(y, \eta, \lambda),
\end{aligned}
$$


where $p_{0}$ and $h_{0}$ are as in Remark 1.3.2, here in the version with $(\eta, \lambda)$ in place of $\eta$, and $\omega, \widetilde{\omega}, \chi$ and $\widetilde{\chi}$ as in formula (1.3.3). Furthermore,

$$
\begin{aligned}
\sigma_{\wedge}\left(m_{j \alpha}\right)(y, \eta, \lambda):= & r^{-\mu} \omega_{0}(r|\eta, \lambda|) r^{j}\left\{\operatorname{op}_{M}^{\gamma_{j}-\frac{n}{2}}\left(f_{j \alpha}\right)(y)\right. \\
& \left.+\operatorname{op}_{M}^{\widetilde{\gamma}-\frac{n}{2}}\left(\widetilde{f}_{j \alpha}\right)(y)\right\}(\eta, \lambda)^{\alpha} \omega_{1}(r|\eta, \lambda|),
\end{aligned}
$$

cf. the $(\eta, \lambda)$-version of (1.2.6), while $\sigma_{\wedge}\left(g_{11}\right)(y, \eta, \lambda)$ is the homogeneous principal symbol of order $\mu$ of $g_{11}$ as a symbol in $S_{\mathrm{cl}}^{\mu}\left(\Omega \times \mathbb{R}^{q+l} ; \mathcal{K}^{s, \gamma}\left(X^{\wedge}\right) \otimes \mathbb{C}^{e}\right.$, $\left.\mathcal{K}^{\infty, \gamma-\mu}\left(X^{\wedge}\right) \otimes \mathbb{C}^{f}\right)$. The remaining entries of (1.4.21) are the homogeneous principal symbols of $g(y, \eta, \lambda)=\left(g_{i j}(y, \eta, \lambda)\right)_{i, j=1,2}$ for $i+j>2$. They are analogously defined as $\sigma_{\wedge}\left(g_{11}\right)(y, \eta, \lambda)$ because $g_{i j}(y, \eta, \lambda)$ are all classical symbols, though with "Douglis-Nirenberg orders", cf. Definition 1.1.1, that cause the extra powers of $\delta$ in the group actions (1.4.22).

Let us write

$$
\sigma(\mathcal{A})=\left(\sigma_{\psi}(\mathcal{A}), \sigma_{\wedge}(\mathcal{A})\right)
$$

called the principal symbol of the operator $\mathcal{A}$.

Remark 1.4.6. Let $\mathcal{A} \in \mathcal{Y}^{\mu}\left(\mathbb{W}, \boldsymbol{g} ; \boldsymbol{v} ; \mathbb{R}^{l}\right)$, and assume that $\mathbb{W}$ is compact. Then $\sigma(\mathcal{A})=0$ implies that

$$
\mathcal{A}(\lambda)=: \mathcal{W}^{s, \gamma}(\mathbb{W} ; \boldsymbol{m}) \rightarrow \mathcal{W}^{s-\mu, \gamma-\mu}(\mathbb{W} ; \boldsymbol{n})
$$

is a compact operator for every $s \in \mathbb{R}$ and $\lambda \in \mathbb{R}^{l}$.

This follows from the fact that $\mathcal{A}(\lambda)$ maps to $\mathcal{W}^{s-\mu+1, \gamma-\mu+\varepsilon}(\mathbb{W} ; \boldsymbol{n})$ for some $\varepsilon>0$, and from the compactness of embeddings $\mathcal{W}^{s^{\prime}, \gamma^{\prime}}(\mathbb{W}, \boldsymbol{n}) \hookrightarrow \mathcal{W}^{s, \gamma}(\mathbb{W}$; n) for $s^{\prime}>s, \gamma^{\prime}>\gamma$.

Let $\mathcal{M}_{\left(\varphi, \varphi^{\prime}\right)}$ denote the operator of multiplication by $\operatorname{diag}\left(\varphi, \varphi^{\prime}\right)$ for $\varphi \in$ $C^{\infty}(\mathbb{W}), \varphi^{\prime} \in C^{\infty}(Y)$. For the case $\left.\varphi\right|_{\partial \mathbb{W}}=\pi^{*} \varphi^{\prime}$, where $\pi: \partial \mathbb{W} \rightarrow Y$ is the canonical projection, we simply write $\mathcal{M}_{\varphi}$. It is easy to verify that $\mathcal{A} \in$ $\mathcal{Y}^{\mu}\left(\mathbb{W}, \boldsymbol{g} ; \boldsymbol{v} ; \mathbb{R}^{l}\right)$ implies $\mathcal{M}_{\left(\varphi, \varphi^{\prime}\right)} \mathcal{A} \mathcal{M}_{\left(\psi, \psi^{\prime}\right)} \in \mathcal{Y}^{\mu}\left(\mathbb{W}, \boldsymbol{g} ; \boldsymbol{v} ; \mathbb{R}^{l}\right)$ for arbitrary $\varphi, \psi \in$ $C^{\infty}(\mathbb{W}), \varphi^{\prime}, \psi^{\prime} \in C^{\infty}(Y)$.

Theorem 1.4.7 ([29, Section 3.4.4]). Let $\mathcal{A} \in \mathcal{Y}^{\mu}\left(\mathbb{W}, \boldsymbol{g} ; \boldsymbol{v} ; \mathbb{R}^{l}\right), \mathcal{B} \in$ $\mathcal{Y}^{\nu}\left(\mathbb{W}, \widetilde{\boldsymbol{g}} ; \widetilde{\boldsymbol{v}} ; \mathbb{R}^{l}\right)$ for $\mu, \nu \in \mathbb{R}$ and

$$
\begin{array}{ll}
\boldsymbol{g}=(\gamma-\nu, \gamma-(\mu+\nu) ; \Theta), & \boldsymbol{v}=\left(E_{0}, F ; J_{0}, J_{+}\right), \\
\widetilde{\boldsymbol{g}}=(\gamma, \gamma-\nu ; \Theta), & \widetilde{\boldsymbol{v}}=\left(E, E_{0} ; J_{-}, J_{0}\right),
\end{array}
$$


and let $\varphi \in C_{0}^{\infty}(\mathbb{W})$. Then we have $\mathcal{A} \mathcal{M}_{\varphi} \mathcal{B} \in \mathcal{Y}^{\mu+\nu}\left(\mathbb{W}, \boldsymbol{h} ; \boldsymbol{w} ; \mathbb{R}^{l}\right)$ for

$$
\boldsymbol{h}=(\gamma, \gamma-(\mu+\nu), \Theta), \quad \boldsymbol{w}=\left(E, F ; J_{-}, J_{+}\right),
$$

and

$$
\sigma\left(\mathcal{A M} \mathcal{M}_{\varphi} \mathcal{B}\right)=\sigma(\mathcal{A}) \sigma\left(\mathcal{M}_{\varphi} \mathcal{B}\right)
$$

(with componentwise multiplication). If $\mathcal{A}$ or $\mathcal{B}$ belong to the space with subscript $M+G$ or $G$, then the same is true of the composition.

Definition 1.4.8. An operator $\mathcal{A} \in \mathcal{Y}^{\mu}\left(\mathbb{W}, \boldsymbol{g} ; \boldsymbol{v} ; \mathbb{R}^{l}\right)$ for

$$
\boldsymbol{g}=(\gamma, \gamma-\mu, \Theta), \quad \boldsymbol{v}=\left(E, F ; J_{-}, J_{+}\right)
$$

is said to be elliptic if

(i) $\mathcal{A}$ is $\sigma_{\psi}$-elliptic, that is, (1.4.20) is an isomorphism,

(ii) $\mathcal{A}$ is $\sigma_{\wedge}$-elliptic, that is, (1.4.21) is an isomorphism for some $s=s_{0} \in \mathbb{R}$.

Clearly, if (1.4.20) is an isomorphism, so is (1.4.18). Moreover, if (1.4.21) is an isomorphism for $s=s_{0}$, then it is an isomorphism for all $s \in \mathbb{R}$.

Note that when (1.4.21) is an isomorphism,

$$
\text { u. l. c. } \sigma_{\wedge}(\mathcal{A})(y, \eta, \lambda): \pi_{Y}^{*} \mathcal{K}^{s, \gamma}\left(X^{\wedge}\right) \otimes E^{\prime} \rightarrow \pi_{Y}^{*} \mathcal{K}^{s-\mu, \gamma-\mu}\left(X^{\wedge}\right) \otimes F^{\prime}
$$

is a family of Fredholm operators. (1.4.29) belongs to the cone algebra on $X^{\wedge}$ for every $(y, \eta, \lambda) \in\left(T^{*} Y \times \mathbb{R}^{l}\right) \backslash 0$. As such it has a "subordinate" principal conormal symbol, namely

$$
\sigma_{M}(\mathcal{A})(y, z): H^{s}\left(X, E_{0}\right) \rightarrow H^{s-\mu}\left(X, F_{0}\right)
$$

that is meromorphic in $z \in \mathbb{C}$ and a family of isomorphisms for all $y \in Y$ and $z \in \Gamma_{(n+1) / 2-\gamma}$.

Theorem 1.4.9 ([29, Section 3.5.2] and [4]). Let $\mathcal{A} \in \mathcal{Y}^{\mu}\left(\mathbb{W}, \boldsymbol{g} ; \boldsymbol{v} ; \mathbb{R}^{l}\right)$ be elliptic. Then $\mathcal{A}$ has a parametrix $\mathcal{P} \in \mathcal{Y}^{-\mu}\left(\mathbb{W}, \boldsymbol{g}^{-1} ; \boldsymbol{v}^{-1} ; \mathbb{R}^{l}\right)$ in the sense that

$$
\begin{aligned}
& \mathcal{M}_{\varphi}-\mathcal{P} \mathcal{M}_{\varphi} \mathcal{A} \mathcal{M}_{\psi} \in \mathcal{Y}^{-\infty}\left(\mathbb{W}, g_{l} ; \boldsymbol{v}_{l} ; \mathbb{R}^{l}\right) \\
& \mathcal{M}_{\varphi}-\mathcal{A M}_{\psi} \mathcal{P} \mathcal{M}_{\varphi} \in \mathcal{Y}^{-\infty}\left(\mathbb{W}, \boldsymbol{g}_{r} ; \boldsymbol{v}_{r} ; \mathbb{R}^{l}\right)
\end{aligned}
$$

holds for every $\varphi, \psi \in C_{0}^{\infty}(\mathbb{W})$ where $\varphi \psi=\varphi$; here $\boldsymbol{g}^{-1}=(\gamma-\mu, \gamma, \Theta)$, $\boldsymbol{v}^{-1}=$ $\left(F, E ; J_{+}, J_{-}\right)$, and $\boldsymbol{g}_{l}=(\gamma, \gamma, \Theta), \boldsymbol{v}_{l}=\left(E, E ; J_{-}, J_{-}\right), \boldsymbol{g}_{r}=(\gamma-\mu, \gamma-\mu, \Theta)$, $\boldsymbol{v}_{r}=\left(F, F ; J_{+}, J_{+}\right)$. 
Corollary 1.4.10. Let $\mathbb{W}$ be compact and $\mathcal{A} \in \mathcal{Y}^{\mu}\left(\mathbb{W}, \boldsymbol{g} ; \boldsymbol{v} ; \mathbb{R}^{l}\right)$ elliptic. Then, in the notation of Theorem 1.4.9, there is a parametrix $\mathcal{P} \in \mathcal{Y}^{-\mu}\left(\mathbb{W}, \boldsymbol{g}^{-1}\right.$; $\left.\boldsymbol{v}^{-1} ; \mathbb{R}^{l}\right)$, where $\mathcal{I}-\mathcal{P} \mathcal{A}=: \mathcal{C}_{l}$ and $\mathcal{I}-\mathcal{A P}=: \mathcal{C}_{r}$ belong to the respective spaces of operators of order $-\infty$. Moreover,

$$
\mathcal{A}(\lambda): \mathcal{W}^{s, \gamma}(\mathbb{W} ; \boldsymbol{m}) \rightarrow \mathcal{W}^{s-\mu, \gamma-\mu}(\mathbb{W} ; \boldsymbol{n})
$$

is a Fredholm operator for every $s \in \mathbb{R}, \lambda \in \mathbb{R}^{l}$, cf. the notation of Remark 1.4.6.

Remark 1.4.11. For $l>0$ the operators (1.4.30) are of index zero for all $s \in \mathbb{R}, \lambda \in \mathbb{R}^{l}$, and there is a constant $c>0$ such that (1.4.30) are isomorphisms for all $s \in \mathbb{R},|\lambda|>c$, and the parametrix $\mathcal{P}$ can be chosen in such a way that $\mathcal{C}_{l}$ and $\mathcal{C}_{r}$ vanish for $|\lambda|>c$.

\section{$\S 2$. Corner Symbols and Iterated Asymptotics}

\section{§2.1. Holomorphic corner symbols}

In this section we assume $\mathbb{W}$ to be compact and consider $\mathcal{Y}^{\mu}\left(\mathbb{W}, \boldsymbol{g} ; \boldsymbol{v} ; \mathbb{R}^{l+1}\right)$ with parameters $(\lambda, \tau) \in \mathbb{R}^{l+1}$; here, $\boldsymbol{g}=(\gamma, \gamma-\mu, \Theta)$ and $\boldsymbol{v}=\left(E, F ; J_{-}, J_{+}\right)$. In our applications we shall interpret $\tau \in \mathbb{R}$ as $\operatorname{Im} w$ for $w \in \Gamma_{\beta}$ with some $\beta$. The corresponding space of parameter-dependent operators will be denoted $\mathcal{Y}^{\mu}\left(\mathbb{W}, \boldsymbol{g} ; \boldsymbol{v} ; \mathbb{R}^{l} \times \Gamma_{\beta}\right)$.

The elements $a(\lambda, w)$ in that space play the role of operator-valued symbols (therefore, we now employ small letters). By Remark 1.4.5 every $a(\lambda, w)$ belongs to a Fréchet subspace

$$
\mathcal{Y}^{\mu}\left(\mathbb{W}, \boldsymbol{g} ; \boldsymbol{v} ; \mathbb{R}^{l} \times \Gamma_{\beta}\right)_{R}
$$

of $\mathcal{Y}^{\mu}\left(\mathbb{W}, \boldsymbol{g} ; \boldsymbol{v} ; \mathbb{R}^{l} \times \Gamma_{\beta}\right)$ for some $R \in \mathbf{A s}_{M+G}(X, \boldsymbol{g} ; \boldsymbol{v})$.

Definition 2.1.1. Let $\mathcal{M}_{R, \mathcal{O}}^{\mu}\left(\mathbb{W}, \boldsymbol{g} ; \boldsymbol{v} ; \mathbb{R}^{l}\right)$ for $R \in \mathbf{A s}_{M+G}(X, \boldsymbol{g} ; \boldsymbol{v}), \boldsymbol{g}$ $=(\gamma, \gamma-\mu, \Theta)$, denote the subspace of all $h(\lambda, w) \in \mathcal{A}\left(\mathbb{C}, \mathcal{Y}^{\mu}\left(\mathbb{W}, \boldsymbol{g} ; \boldsymbol{v} ; \mathbb{R}_{\lambda}^{l}\right)_{R}\right)$ such that

$$
h(\lambda, w)_{\mathbb{R}^{l} \times \Gamma_{\beta}} \in \mathcal{Y}^{\mu}\left(\mathbb{W}, \boldsymbol{g} ; \boldsymbol{v} ; \mathbb{R}^{l} \times \Gamma_{\beta}\right)_{R}
$$

for every $\beta \in \mathbb{R}$, uniformly in $c \leq \beta \leq c^{\prime}$ for arbitrary $c \leq c^{\prime}$. Moreover, we define $\mathcal{M}_{R, \mathcal{O}}^{-\infty}\left(\mathbb{W}, \boldsymbol{g} ; \boldsymbol{v} ; \mathbb{R}^{l}\right)$ for $\boldsymbol{g}=(\gamma, \gamma-\mu, \Theta)$ to be the subspace of all $h(\lambda, w) \in$ $\mathcal{A}\left(\mathbb{C}, \mathcal{Y}^{-\infty}\left(\mathbb{W}, \boldsymbol{g} ; \boldsymbol{v} ; \mathbb{R}^{l}\right)_{R}\right)$ such that $\left.h(\lambda, w)\right|_{\mathbb{R}^{l} \times \Gamma_{\beta}} \in \mathcal{S}\left(\Gamma_{\beta}, \mathcal{Y}^{-\infty}\left(\mathbb{W}, \boldsymbol{g} ; \boldsymbol{v} ; \mathbb{R}^{l}\right)_{R}\right)$ for every $\beta \in \mathbb{R}$, uniformly in $c \leq \beta \leq c^{\prime}$ for arbitrary $c \leq c^{\prime}$. 
The spaces $\mathcal{M}_{R, \mathcal{O}}^{\mu}\left(\mathbb{W}, \boldsymbol{g} ; \boldsymbol{v}, \mathbb{R}^{l}\right)$ and $\mathcal{M}_{R, \mathcal{O}}^{-\infty}\left(\mathbb{W}, \boldsymbol{g} ; \boldsymbol{v} ; \mathbb{R}^{l}\right)$ are Fréchet in a canonical way. In this paper we are mainly interested in the case $l=0$; then we simply omit $\mathbb{R}^{l}$ in the notation.

The spaces of Definition 2.1.1 have been introduced by Maniccia and Schulze [16]; most of the results of Sections 2.1. and 2.2 are proved there.

Set $f(\lambda, \tau):=a(\lambda, \beta+i \tau)$ for fixed $\beta$, and define $k(f ; \lambda, \zeta):=\left(F_{\tau \rightarrow \zeta}^{-1} f\right)(\lambda$, $\zeta$ ), where $F$ is the one-dimensional Fourier transform. Choose any $\varphi(\zeta) \in$ $C_{0}^{\infty}(\mathbb{R})$, form

$$
(H(\varphi) f)(\lambda, \tau):=F_{\zeta \rightarrow \tau}\{\varphi(\zeta) k(f ; \lambda, \zeta)\}
$$

and set $h_{1}(\lambda, \beta+i \tau):=(H(\varphi) f)(\lambda, \tau)$. We also write $h_{1}(\lambda, w)=(H(\varphi) a)(\lambda, w)$ for $f(\lambda, \tau)=a(\lambda, \beta+i \tau)$.

In the sequel we fix any choice of asymptotic data $R$.

Theorem 2.1.2. For every $\varphi \in C_{0}^{\infty}(\mathbb{R})$ and $a(\lambda, w) \in \mathcal{Y}^{\mu}\left(\mathbb{W}, \boldsymbol{g} ; \boldsymbol{v} ; \mathbb{R}^{l} \times\right.$ $\left.\Gamma_{\beta}\right)_{R}$ we have $h_{1}(\lambda, w) \in \mathcal{Y}^{\mu}\left(\mathbb{W}, \boldsymbol{g} ; \boldsymbol{v} ; \mathbb{R}^{l} \times \Gamma_{\beta}\right)_{R}$. Moreover, there is an $h(\lambda, w) \in$ $\mathcal{M}_{R, \mathcal{O}}^{\mu}\left(\mathbb{W}, \boldsymbol{g} ; \boldsymbol{v} ; \mathbb{R}^{l}\right)$ (that is, of course, unique) such that

$$
h_{1}(\lambda, w)=\left.h(\lambda, w)\right|_{\mathbb{R}^{l} \times \Gamma_{\beta}} .
$$

In other words, $h(\lambda, w)$ is the analytic extension of $h_{1}(\lambda, w)$ with respect to $w$ from $\Gamma_{\beta}$ to $\mathbb{C}$. We then set $h(\lambda, w):=H(\varphi)(a)(\lambda, w), w \in \mathbb{C}$.

Theorem 2.1.3. For every $h(\lambda, w) \in \mathcal{M}_{R, \mathcal{O}}^{\mu}\left(\mathbb{W}, \boldsymbol{g} ; \boldsymbol{v} ; \mathbb{R}^{l}\right)$ and arbitrary $\alpha, \beta \in \mathbb{R}$ we have

$$
\sigma(h(\lambda, \alpha+i \tau))=\sigma(h(\lambda, \beta+i \tau))
$$

cf. formula (1.4.25); here $h(\lambda, \alpha+i \tau), \alpha \in \mathbb{R}$, is regarded as an element of $\mathcal{Y}^{\mu}\left(\mathbb{W}, \boldsymbol{g} ; \boldsymbol{v} ; \mathbb{R}^{l+1}\right)_{R}$.

Remark 2.1.4. Let $h(\lambda, w) \in \mathcal{M}_{R, \mathcal{O}}^{\mu}\left(\mathbb{W}, \boldsymbol{g} ; \boldsymbol{v} ; \mathbb{R}^{l}\right)$, and assume that $h(\lambda$, $\beta+i \tau) \in \mathcal{Y}_{R}^{-\infty}\left(\mathbb{W}, \boldsymbol{g} ; \boldsymbol{v} ; \mathbb{R}^{l}\right)$ for some fixed $\beta \in \mathbb{R}$. Then $h(\lambda, w) \in \mathcal{M}_{R, \mathcal{O}}^{-\infty}(\mathbb{W}, \boldsymbol{g} ; \boldsymbol{v} ;$ $\left.\mathbb{R}^{l}\right)$.

Theorem 2.1.5. For every $a(\lambda, w) \in \mathcal{Y}^{\mu}\left(\mathbb{W}, \boldsymbol{g} ; \boldsymbol{v} ; \mathbb{R}^{l} \times \Gamma_{\beta}\right)_{R}$ there exists an $h(\lambda, w) \in \mathcal{M}_{R, \mathcal{O}}^{\mu}\left(\mathbb{W}, \boldsymbol{g} ; \boldsymbol{v} ; \mathbb{R}^{l}\right)$ such that

$$
a(\lambda, w)=\left.h(\lambda, w)\right|_{\mathbb{R}^{l} \times \Gamma_{\beta}} \bmod \mathcal{Y}^{-\infty}\left(\mathbb{W}, \boldsymbol{g} ; \boldsymbol{v} ; \mathbb{R}^{l} \times \Gamma_{\beta}\right)_{R} .
$$

To construct $h(\lambda, w)$ in Theorem 2.1.5 it suffices to set

$$
h(\lambda, w)=H(\psi) a(\lambda, w)
$$


for any $\psi(\zeta) \in C_{0}^{\infty}(\mathbb{R})$ such that $\psi(\zeta)=1$ in a neighbourhood of $\zeta=0$. In particular, we have $H(\psi-\widetilde{\psi}) a(\lambda, w) \in \mathcal{M}_{R, \mathcal{O}}^{-\infty}\left(\mathbb{W}, \boldsymbol{g} ; \boldsymbol{v} ; \mathbb{R}^{l}\right)$ for two such cut-off functions $\psi$ and $\widetilde{\psi}$.

Notice that there is a formal similarity between Theorem 2.1.5 and Theorem 1.3.1. Formula (2.1.2) is just what we called a kernel cut-off construction. The important point here is that despite of the complexity of parameterdependent edge operators the kernel cut-off operators $H(\varphi), H(\psi)$ preserve all data, including asymptotics, but produce holomorphic families with the properties in Definition 2.1.1. An analogous construction in a simpler situation has been applied in [27], see also the author's joint paper with Fedosov and Tarkhanov [7].

Remark 2.1.6. Since kernel cut-off operators only act with respect covariables, Theorem 2.1.5 extends to the case of operator families that smoothly depend on other variables. For instance, we will apply below the fact that for $a(t, \lambda, w) \in \mathcal{Y}^{\mu}\left(\mathbb{W}, \boldsymbol{g} ; \boldsymbol{v} ; \mathbb{R}^{l} \times \Gamma_{\beta}\right)_{R}$ there exists an $h(t, \lambda, w) \in C^{\infty}\left(\overline{\mathbb{R}}_{+}, \mathcal{M}_{R, \mathcal{O}}^{\mu}(\mathbb{W}\right.$, $\left.\left.\boldsymbol{g} ; \boldsymbol{v} ; \mathbb{R}^{l}\right)\right)$ such that

$$
a(t, \lambda, w)-\left.h(t, \lambda, w)\right|_{\overline{\mathbb{R}}_{+} \times \mathbb{R}^{l} \times \Gamma_{\beta}} \in C^{\infty}\left(\overline{\mathbb{R}}_{+}, \mathcal{Y}^{-\infty}\left(\mathbb{W}, \boldsymbol{g} ; \boldsymbol{v} ; \mathbb{R}^{l} \times \Gamma_{\beta}\right)_{R}\right) .
$$

\section{$\S 2.2$. Meromorphic corner symbols and ellipticity}

An element $h(\lambda, w) \in \mathcal{M}_{R, \mathcal{O}}^{\mu}\left(\mathbb{W}, \boldsymbol{g} ; \boldsymbol{v} ; \mathbb{R}^{l}\right), R \in \mathbf{A s}_{M+G}(X, \boldsymbol{g} ; \boldsymbol{v})$, is said to be elliptic, if there is a $\beta \in \mathbb{R}$ such that $h(\lambda, \beta+i \tau)$ is elliptic in the space $\mathcal{Y}^{\mu}\left(\mathbb{W}, \boldsymbol{g} ; \boldsymbol{v} ; \mathbb{R}_{\lambda, \tau}^{l+1}\right)$, cf. Definition 1.4.8. From Theorem 2.1.3 we know that then $h(\lambda, \beta+i \tau)$ is elliptic for arbitrary $\beta \in \mathbb{R}$, i.e., the definition is independent of the choice of $\beta$. Let $h(\lambda, w) \in \mathcal{M}_{R, \mathcal{O}}^{\mu}\left(\mathbb{W}, \boldsymbol{g} ; \boldsymbol{v} ; \mathbb{R}^{l}\right)$ be elliptic. In view of Remark 1.4.11 the operators

$$
h(\lambda, w): \mathcal{W}^{s, \gamma}(\mathbb{W} ; \boldsymbol{m}) \rightarrow \mathcal{W}^{s-\mu, \gamma-\mu}(\mathbb{W} ; \boldsymbol{n})
$$

are Fredholm and of index zero for all $s$, and they are isomorphisms for all $|\lambda, w| \geq c$ for some $c>0$. Let us now set $l=0$, i.e., $\lambda$ disappears. Then, for every $\alpha<\alpha^{\prime}$ there is an $I\left(\alpha, \alpha^{\prime}\right)$ such that the operators (2.2.1) are isomorphisms for all $w \in \mathbb{C}$ with $\alpha \leq \operatorname{Re} w \leq \alpha^{\prime}$ and $|\operatorname{Im} w| \geq I\left(\alpha, \alpha^{\prime}\right)$. We are now in a context that is familiar in the abstract set up of holomorphic Fredholm functions in Hilbert spaces, cf. Gohberg and Sigal [10]. Here, we have more specific information.

Remark 2.2.1. Let $h(w) \in \mathcal{M}_{R, \mathcal{O}}^{\mu}(\mathbb{W}, \boldsymbol{g} ; \boldsymbol{v})$ be elliptic. Then there is a countable set $D \subset \mathbb{C}$ such that $D \cap\left\{w: \alpha \leq \operatorname{Re} w \leq \alpha^{\prime}\right\}$ is finite for every 
$\alpha \leq \alpha^{\prime}$, where the operators

$$
h(w): \mathcal{W}^{s, \gamma}(\mathbb{W} ; \boldsymbol{m}) \rightarrow \mathcal{W}^{s-\mu, \gamma-\mu}(\mathbb{W} ; \boldsymbol{n})
$$

are isomorphisms for all $w \in \mathbb{C} \backslash D$ and all $s \in \mathbb{R}$, and there is an $S \in$ $\operatorname{As}\left(X, \boldsymbol{g}^{-1} ; \boldsymbol{v}^{-1}\right)$ such that $h^{-1}(w) \in \mathcal{A}\left(\mathbb{C} \backslash D, \mathcal{Y}^{-\mu}\left(\mathbb{W}, \boldsymbol{g}^{-1} ; \boldsymbol{v}^{-1}\right)_{S}\right)$, cf. the notation in Theorem 1.4.9.

As is known from abstract Fredholm functions, $h^{-1}(w)$ extends to a meromorphic Fredholm function with poles $d_{j} \in D$ and multiplicities $n_{j}+1, n_{j} \in \mathbb{N}$, $j \in \mathbb{Z}$, and Laurent coefficients at $\left(w-d_{j}\right)^{-(k+1)}, 0 \leq k \leq n_{j}$, that are operators of finite rank. These can be characterised in our concrete situation as elements in $\mathcal{Y}^{-\infty}\left(\mathbb{W}, \boldsymbol{g}^{-1} ; \boldsymbol{v}^{-1}\right)_{S}$.

To single out some convenient spaces of meromorphic operator functions we introduce the set $\mathbf{A} \mathbf{s}^{\bullet}(\mathbb{W} ; \boldsymbol{v})_{S}$ of sequences

$$
T:=\left\{\left(d_{j}, n_{j}, L_{j}\right)\right\}_{j \in \mathbb{Z}},
$$

so-called discrete corner asymptotic types (associated with $S \in \mathbf{A s}_{M+G}(X, \boldsymbol{g}$; $\boldsymbol{v})$, weight data $\boldsymbol{g}=(\delta, \delta-\mu, \Theta)$ and $\boldsymbol{v}=\left(H, L ; G_{-}, G_{+}\right)$, for bundles $H, L$ on $\mathbb{W}$ and $G_{-}, G_{+}$on $Y$ ), where we assume $\pi_{\mathbb{C}} T \cap\left\{w: c \leq \operatorname{Re} w \leq c^{\prime}\right\}$ to be finite for every $c \leq c^{\prime}, \pi_{\mathbb{C}} T:=\left\{d_{j}\right\}_{j \in \mathbb{Z}}$, and $L_{j}$ to be a finite-dimensional subspace of $\mathcal{Y}^{-\infty}(\mathbb{W}, \boldsymbol{g} ; \boldsymbol{v})_{S}$ of operators of finite rank for all $j$.

Definition 2.2.2. $\quad \mathcal{M}_{S, T}^{-\infty}(\mathbb{W}, \boldsymbol{g} ; \boldsymbol{v})$ for $S \in \mathbf{A s}_{M+G}(X, \boldsymbol{g} ; \boldsymbol{v})$ and $T \in$ $\mathbf{A s}^{\bullet}(\mathbb{W} ; \boldsymbol{v})_{S}$ denotes the set of all

$$
f(w) \in \mathcal{A}\left(\mathbb{C} \backslash \pi_{\mathbb{C}} T, \mathcal{Y}^{-\infty}(\mathbb{W}, \boldsymbol{g} ; \boldsymbol{v})_{S}\right)
$$

that are meromorphic with poles at $d_{j} \in \pi_{\mathbb{C}} T$ of multiplicities $n_{j}+1$ and Laurent coefficients at $\left(w-d_{j}\right)^{-(k+1)}$ belonging to $L_{j}$ for $0 \leq k \leq n_{j}, j \in \mathbb{Z}$, where $\left.\chi(w) f(w)\right|_{\Gamma_{\beta}} \in \mathcal{Y}^{-\infty}\left(\mathbb{W}, \boldsymbol{g} ; \boldsymbol{v} ; \Gamma_{\beta}\right)_{S}$ for every $\beta \in \mathbb{R}$, uniformly in $c \leq \beta \leq c^{\prime}$ for arbitrary $c \leq c^{\prime}$, for any $\pi_{\mathbb{C}} T$-excision function $\chi$ (i.e., $\chi \in C^{\infty}(\mathbb{C}), \chi(w)=0$ for $\operatorname{dist}\left(w, \pi_{\mathbb{C}} T\right)<\varepsilon_{0}, \chi(w)=1$ for $\operatorname{dist}\left(w, \pi_{\mathbb{C}} T\right)>\varepsilon_{1}$, for certain $\left.0<\varepsilon_{0}<\varepsilon_{1}\right)$.

The space $\mathcal{M}_{S, T}^{-\infty}(\mathbb{W}, \boldsymbol{g} ; \boldsymbol{v})$ is Fréchet in a canonical way. For arbitrary $\mu \in \mathbb{R}, \boldsymbol{g}=(\delta, \delta-\mu, \Theta)$, we set

$$
\mathcal{M}_{S, T}^{\mu}(\mathbb{W}, \boldsymbol{g} ; \boldsymbol{v}):=\mathcal{M}_{S, \mathcal{O}}^{\mu}(\mathbb{W}, \boldsymbol{g} ; \boldsymbol{v})+\mathcal{M}_{S, T}^{-\infty}(\mathbb{W}, \boldsymbol{g} ; \boldsymbol{v}),
$$

endowed with the Fréchet topology of the non-direct sum. 
Theorem 2.2.3. Let $a(w) \in \mathcal{M}_{R, V}^{\mu}(\mathbb{W}, \boldsymbol{g} ; \boldsymbol{v}), b(w) \in \mathcal{M}_{\widetilde{R}, \widetilde{V}}^{\nu}(\mathbb{W}, \widetilde{\boldsymbol{g}} ; \widetilde{\boldsymbol{v}})$ for any pairs of asymptotic types

$$
\begin{aligned}
& (R, V) \in \mathbf{A} \mathbf{s}_{M+G}(X, \boldsymbol{g} ; \boldsymbol{v}) \times \mathbf{A} \mathbf{s}^{\bullet}(\mathbb{W} ; \boldsymbol{v})_{R}, \\
& (\widetilde{R}, \widetilde{V}) \in \mathbf{A} \mathbf{s}_{M+G}(X, \widetilde{\boldsymbol{g}} ; \widetilde{\boldsymbol{v}}) \times \mathbf{A} \mathbf{s}^{\bullet}(\mathbb{W} ; \widetilde{\boldsymbol{v}})_{\widetilde{R}},
\end{aligned}
$$

with (1.4.26), (1.4.27). Then we have $(a b)(w) \in \mathcal{M}_{S, T}^{\mu+\nu}(\mathbb{W}, \boldsymbol{h} ; \boldsymbol{w})$ for a resulting pair $(S, T) \in \mathbf{A s}_{M+G}(X, \boldsymbol{h} ; \boldsymbol{w}) \times \mathbf{A} \mathbf{s}^{\bullet}(\mathbb{W} ; \boldsymbol{w})_{S}$, where $\boldsymbol{h}$ and $\boldsymbol{w}$ are given as in (1.4.28).

The proof of Theorem 2.2.3 employs Theorem 1.4.7 above in various ways, in particular, for the characterisation of resulting asymptotic types under compositions.

An element $f \in \mathcal{M}_{S, T}^{\mu}(\mathbb{W}, \boldsymbol{g} ; \boldsymbol{v})$ is said to be elliptic, if for any decomposition $f=h+m$ for $\mathcal{M}_{S, \mathcal{O}}^{\mu}(\mathbb{W}, \boldsymbol{g} ; \boldsymbol{v}), m \in \mathcal{M}_{S, T}^{-\infty}(\mathbb{W}, \boldsymbol{g} ; \boldsymbol{v})$, the element $h$ is elliptic in the above-mentioned sense (this does not depend on the choice of the decomposition). Equivalently, we can require the family $\left.f\right|_{\Gamma_{\beta}}$ to be elliptic in $\mathcal{Y}^{\mu}\left(\mathbb{W}, \boldsymbol{g} ; \boldsymbol{v} ; \Gamma_{\beta}\right)_{S}$ for any $\beta \in \mathbb{R}$ such that $\pi_{\mathbb{C}} T \cap \Gamma_{\beta}=\emptyset$ (which is then independent of $\beta$ ).

The following theorem extends Remark 2.2.1 to the case of elliptic meromorphic Mellin symbols. It will be crucial for the following regularity results with edge-corner asymptotics below.

Theorem 2.2.4. Let $f \in \mathcal{M}_{R, V}^{\mu}(\mathbb{W}, \boldsymbol{g} ; \boldsymbol{v})$ for

$$
R \in \mathbf{A s}_{M+G}(X, \boldsymbol{g} ; \boldsymbol{v}), \quad V \in \mathbf{A} \mathbf{s}^{\bullet}(\mathbb{W} ; \boldsymbol{v})_{R},
$$

be elliptic. Then there is an inverse $f^{-1}(w) \in \mathcal{A}\left(\mathbb{C} \backslash \pi_{\mathbb{C}} T, \mathcal{Y}^{-\mu}\left(\mathbb{W}, \boldsymbol{g}^{-1} ; \boldsymbol{v}^{-1}\right)_{S}\right)$ for certain $S \in \mathbf{A s}_{M+G}\left(X, \boldsymbol{g}^{-1} ; \boldsymbol{v}^{-1}\right), T \in \mathbf{A} \mathbf{s}^{\bullet}\left(\mathbb{W} ; \boldsymbol{v}^{-1}\right)_{S}$, that extends to an element $f^{-1} \in \mathcal{M}_{S, T}^{-\mu}\left(\mathbb{W}, \boldsymbol{g}^{-1} ; \boldsymbol{v}^{-1}\right)$ (in the sense of the multiplication from Theorem 2.2.3).

Proof. First, if $f(w) \in \mathcal{M}_{R, V}^{\mu}(\mathbb{W}, \boldsymbol{g} ; \boldsymbol{v})$ is elliptic, there is a countable set $D \subset \mathbb{C}$ such that $D \cap K$ is finite for every compact set $K$ where $K \cap \pi_{\mathbb{C}} V=\emptyset$; in addition, for every $\alpha \leq \alpha^{\prime}$ there exists an $M\left(\alpha, \alpha^{\prime}\right)$ such that $f(w)$ is bijective for all $w \in\left\{w \in \mathbb{C}: \alpha \leq \operatorname{Re} w \leq \alpha^{\prime},|\operatorname{Im} w| \geq M\left(\alpha, \alpha^{\prime}\right)\right\}$. In particular, we find a $\beta \in \mathbb{R}$ such that $\left.f\right|_{\Gamma_{\beta}} \in \mathcal{Y}^{\mu}\left(\mathbb{W}, \boldsymbol{g} ; \boldsymbol{v} ; \Gamma_{\beta}\right)_{R}$ is a parameter-dependent elliptic element. Corollary 1.4.10 gives us a parameter-dependent elliptic parametrix $g \in \mathcal{Y}^{-\mu}\left(\mathbb{W}, \boldsymbol{g}^{-1} ; \boldsymbol{v}^{-1} ; \Gamma_{\beta}\right)_{\widetilde{S}}$ where the existence of $\widetilde{S} \in \mathbf{A s}_{M+G}\left(X, \boldsymbol{g}^{-1} ; \boldsymbol{v}^{-1}\right)$ is an automatic consequence of the definition (recall that edge operator belongs to 
a Fréchet subspace with asymptotics of a certain type $\widetilde{S})$. Applying Theorem 2.1 .5 we get an $h(w) \in \mathcal{M}_{\widetilde{S}, \mathcal{O}}^{-\mu}\left(\mathbb{W}, \boldsymbol{g}^{-1} ; \boldsymbol{v}^{-1}\right)$ such that $\left.h\right|_{\Gamma_{\beta}}=g(w)$ modulo a family of order $-\infty$. In other words, also $\left.h\right|_{\Gamma_{\beta}}$ is a parametrix of $\left.f\right|_{\Gamma_{\beta}}$. Now $\left.\{h(w) f(w)\}\right|_{\Gamma_{\beta}}-1 \in \mathcal{Y}^{-\infty}\left(\mathbb{W}, \boldsymbol{g}_{l} ; \boldsymbol{v}_{l} ; \Gamma_{\beta}\right)$ and $h f \in \mathcal{M}_{\widetilde{S}, \widetilde{V}}^{0}\left(\mathbb{W}, \boldsymbol{g}_{l} ; \boldsymbol{v}_{l}\right)$ implies $l:=$ $h f \in \mathcal{M}_{\widetilde{S}, \widetilde{V}}^{-\infty}\left(\mathbb{W}, \boldsymbol{g}_{l} ; \boldsymbol{v}_{l}\right)$ (the latter relation is an easy consequence of Theorem 2.1.2). In other words we have $h f=1+l$, and it remains to show that $(1+l)^{-1}=1+m$ for some $m \in \mathcal{M}_{\widetilde{S}, \widetilde{V}}^{0}\left(\mathbb{W}, \boldsymbol{g}_{l} ; \boldsymbol{v}_{l}\right)$. However, this is analogous to the methods in the cone theory, see, for instance, [20, Lemma 4.3.13]. Applying once again Theorem 2.2.3 we get $f^{-1}=(1+m) g$ of the desired kind.

\section{$\S 2.3$. Weighted corner Sobolev spaces}

We now introduce a new class of weighted Sobolev spaces on a (stretched) corner $\mathbb{W}^{\wedge}=\mathbb{R}_{+} \times \mathbb{W}$, where $\mathbb{W}$ is a compact (stretched) manifold with edge. First, let $H$ be a Hilbert space with group action $\left\{\kappa_{\lambda}\right\}_{\lambda \in \mathbb{R}_{+}}$, and assume that to $H$ is associated a dimensional number $m \in \mathbb{N}$ that is known and fixed in each concrete case. (The number $m$ is not the dimension of $H$ but rather a number given independently and paired with $H$ ). We then define the space $\mathcal{V}^{s, \delta}\left(\mathbb{R}_{+} \times \mathbb{R}^{q}, H\right)$ for $s, \delta \in \mathbb{R}$, to be the completion of $C_{0}^{\infty}\left(\mathbb{R}_{+} \times \mathbb{R}^{q}, H\right)$ with respect to the norm

$$
\left\{\frac{1}{2 \pi i} \int_{\Gamma_{\frac{m+1}{2}-\delta}} \int_{\mathbb{R}^{q}}\langle w, \eta\rangle^{2 s}\left\|\kappa_{\langle w, \eta\rangle}^{-1}\left(M_{t \rightarrow w} F_{y \rightarrow \eta} u\right)(w, \eta)\right\|_{H}^{2} d w d \eta\right\}^{\frac{1}{2}} .
$$

Here, $M_{t \rightarrow w}$ is the Mellin transform in $t \in \mathbb{R}_{+}$and $F_{y \rightarrow \eta}$ the Fourier transform in $y \in \mathbb{R}^{q}$.

The choice of the group $\left\{\kappa_{\lambda}\right\}_{\lambda \in \mathbb{R}_{+}}$is assumed to be known and fixed in each concrete case. Let $\mathcal{V}^{s, \delta}\left(\mathbb{R}_{+} \times \mathbb{R}^{q}, H\right)_{1}$ denote for a moment the corresponding space for $\kappa_{\lambda}=\operatorname{id}_{H}$ for all $\lambda \in \mathbb{R}_{+}$. Then the operator $T:=M^{-1} F^{-1} \kappa_{\langle w, \eta\rangle}^{-1} M F$ induces an isomorphism

$$
T: \mathcal{V}^{s, \delta}\left(\mathbb{R}_{+} \times \mathbb{R}^{q}, H\right) \rightarrow \mathcal{V}^{s, \delta}\left(\mathbb{R}_{+} \times \mathbb{R}^{q}, H\right)_{1}
$$

This allows us to single out subspaces of $\mathcal{V}^{s, \delta}\left(\mathbb{R}_{+} \times \mathbb{R}^{q}, H\right)$ connected with subspaces of $E \subset H$, where $\left\{\kappa_{\lambda}\right\}_{\lambda \in \mathbb{R}_{+}}$does not necessarily induce a group action on $E$, namely

$$
T^{-1}\left\{\mathcal{V}^{s, \delta}\left(\mathbb{R}_{+} \times \mathbb{R}^{q}, E\right)_{1}\right\}
$$


Similar considerations make sense when $H$ is a Fréchet space with group action. We then get spaces $\mathcal{V}^{s, \delta}\left(\mathbb{R}_{+} \times \mathbb{R}^{q}, H\right)$ by the same scheme as above in the beginning of Section 1.4.

Applying the identity $M\left(t^{\beta} u\right)(w)=(M u)(w+\beta)$ for arbitrary $\beta \in \mathbb{R}$ we obtain $t^{\beta} \mathcal{V}^{s, \delta}\left(\mathbb{R}_{+} \times \mathbb{R}^{q}, H\right)=\mathcal{V}^{s, \delta+\beta}\left(\mathbb{R}_{+} \times \mathbb{R}^{q}, H\right)$ for all $\beta, \delta \in \mathbb{R}$ and $s \in \mathbb{R}$.

In our applications we shall insert the spaces $H=\mathcal{K}^{s, \gamma}\left(X^{\wedge}\right)$ and Fréchet subspaces $\mathcal{K}_{P}^{s, \gamma}\left(X^{\wedge}\right)$ with asymptotics of type $P \in \operatorname{As}\left(X,(\gamma, \Theta)^{(\bullet)}\right)$, cf. Section 1.1. In this case we set $m=1+n+q$ where $n=\operatorname{dim} X$. In other words, there are the spaces

$$
\mathcal{V}^{s, \delta}\left(\mathbb{R}_{+} \times \mathbb{R}^{q}, \mathcal{K}^{s, \gamma}\left(X^{\wedge}\right)\right) \quad \text { and } \quad \mathcal{V}^{s, \delta}\left(\mathbb{R}_{+} \times \mathbb{R}^{q}, \mathcal{K}_{P}^{s, \gamma}\left(X^{\wedge}\right)\right),
$$

respectively.

To see the nature of singular functions in the spaces $\mathcal{V}^{s, \delta}\left(\mathbb{R}_{+} \times \mathbb{R}^{q}\right.$, $\left.\mathcal{K}_{P}^{s, \gamma}\left(X^{\wedge}\right)\right)$ we first suppose that $P$ is a discrete asymptotic type associated with $(\gamma, \Theta)$ for finite $\Theta$, written as (1.1.6). Let $\mathcal{E}_{P}\left(X^{\wedge}\right)$ denote the linear span of all functions $\omega(r) c_{j k}(x) r^{-p_{j}} \log ^{k} r$ for a fixed cut-off function $\omega(r)$ and arbitrary $c_{j k} \in L_{j}, 0 \leq k \leq m_{j}, 0 \leq j \leq N$. Then we have $\mathcal{K}_{P}^{s, \gamma}\left(X^{\wedge}\right)=$ $\mathcal{K}_{\Theta}^{s, \gamma}\left(X^{\wedge}\right)+\mathcal{E}_{P}\left(X^{\wedge}\right)$ which is a direct decomposition, and it follows a direct decomposition

$$
\begin{aligned}
\mathcal{V}^{s, \delta} & \left(\mathbb{R}_{+} \times \mathbb{R}^{q}, \mathcal{K}_{P}^{s, \gamma}\left(X^{\wedge}\right)\right) \\
& =\mathcal{V}^{s, \delta}\left(\mathbb{R}_{+} \times \mathbb{R}^{q}, \mathcal{K}_{\Theta}^{s, \gamma}\left(X^{\wedge}\right)\right)+T^{-1} \mathcal{V}^{s, \delta}\left(\mathbb{R}_{+} \times \mathbb{R}^{q}, \mathcal{E}_{P}\left(X^{\wedge}\right)\right)_{1} .
\end{aligned}
$$

The first space on the right hand side represents flat remainders of asymptotics, while the elements belonging to $T^{-1}\left\{\mathcal{V}^{s, \delta}\left(\mathbb{R}_{+} \times \mathbb{R}^{q}, \mathcal{E}_{P}\left(X^{\wedge}\right)\right)_{1}\right\}$ are just the singular functions of the edge part of the corner asymptotics. The latter space is the linear span of all elements of the form

$$
M_{\frac{m}{2}+\delta, w \rightarrow t}^{-1} F_{\eta \rightarrow y}^{-1}\left\{\langle w, \eta\rangle^{-\frac{n+1}{2}} \omega(r\langle w, \eta\rangle)(r\langle w, \eta\rangle)^{-p_{j}}(\log r\langle w, \eta\rangle)^{k} \hat{v}_{j k}(w, \eta)\right\}
$$

for $c_{j k} \in L_{j}, v_{j k}(t, y) \in \mathcal{V}^{s, \delta}\left(\mathbb{R}_{+} \times \mathbb{R}^{q}\right), 0 \leq k \leq m_{j}, 0 \leq j \leq N$. Here, $\mathcal{V}^{s, \delta}\left(\mathbb{R}_{+} \times \mathbb{R}^{q}\right):=\mathcal{V}^{s, \delta}\left(\mathbb{R}_{+} \times \mathbb{R}^{q}, \mathbb{C}\right)_{1}$, and $\hat{v}(w, \eta):=M_{m / 2+\delta, t \rightarrow w} F_{y \rightarrow \eta} v(t, y)$. Concerning asymptotic types $P$ we assume, for instance, a representation (1.1.8) for a compact set $K \subset S_{((n+1) / 2-\gamma+\vartheta,(n+1) / 2-\gamma)}$. In this case the space $T^{-1}\left\{\mathcal{V}^{s, \delta}\right.$ $\left.\left(\mathbb{R}_{+} \times \mathbb{R}^{q}, \mathcal{E}_{K}\left(X^{\wedge}\right)\right)_{1}\right\}$ consists of all elements of the form

$$
M_{\frac{m}{2}+\delta, w \rightarrow t}^{-1}\left\{\langle w, \eta\rangle^{-\frac{n+1}{2}} \omega(r\langle w, \eta\rangle)\left\langle\zeta_{z},(r\langle w, \eta\rangle)^{-z}\right\rangle\right\}
$$

for arbitrary $\zeta \in \mathcal{A}^{\prime}\left(K, C^{\infty}\left(X, \hat{\mathcal{V}}^{s, \delta}\left(\mathbb{R}_{+} \times \mathbb{R}^{q}\right)\right)\right.$, where $\hat{\mathcal{V}}^{s, \delta}\left(\mathbb{R}_{+} \times \mathbb{R}^{q}\right):=\{\hat{v}(t, y):$ $\left.v \in \mathcal{V}^{s, \delta}\left(\mathbb{R}_{+} \times \mathbb{R}^{q}\right)\right\}$. 
Let $W$ be a compact manifold with edge $Y, q=\operatorname{dim} Y$, and $\mathbb{W}$ the stretched manifold. By assumption $\partial \mathbb{W}$ has a neighbourhood $\mathbb{V}$ with a global splitting of variables $(r, x, y) \in[0,1) \times X \times Y$.

Choose an open covering $\left\{G_{1}, \ldots, G_{N}\right\}$ of $Y$ by coordinate neighbourhoods and diffeomorphisms $\kappa_{j}: G_{j} \rightarrow \mathbb{R}^{q}$, further a subordinate partition of unity $\left\{\varphi_{1}, \ldots, \varphi_{N}\right\}$, and form the diffeomorphisms $\chi_{j}: \mathbb{R}_{+} \times X \times G_{j} \rightarrow \mathbb{R}_{+} \times X \times \mathbb{R}^{q}$ by setting $\chi_{j}(t, x, y)=\left(t, x, \kappa_{j}(y)\right), j=1, \ldots, N$. We then define the space $\mathcal{V}^{s, \delta}\left(\mathbb{R}_{+} \times Y, \mathcal{K}^{s, \gamma}\left(X^{\wedge}\right)\right)$ for $s, \gamma, \delta \in \mathbb{R}$ to be the completion of $C_{0}^{\infty}\left(\mathbb{R}_{+} \times Y \times X^{\wedge}\right)$ with respect to the norm

$$
\left\{\sum_{j=1}^{N}\left\|\left(\chi_{j}^{-1}\right)^{*}\left(\varphi_{j} u\right)\right\|_{\mathcal{V}^{s, \delta}\left(\mathbb{R}_{+} \times \mathbb{R}^{q}, \mathcal{K}^{s, \gamma}\left(X^{\wedge}\right)\right)}^{2}\right\}^{\frac{1}{2}} .
$$

Clearly, we get an equivalent norm when we change the charts on $Y$ or the partition of unity. In a similar manner we obtain spaces

$$
\mathcal{V}^{s, \delta}\left(\mathbb{R}_{+} \times Y, \mathcal{K}_{P}^{s, \gamma}\left(X^{\wedge}\right)\right):=\lim _{j \in \mathbb{N}} \mathcal{V}^{s, \delta}\left(\mathbb{R}_{+} \times Y, E^{j}\right)
$$

for every $P \in \operatorname{As}(X,(\gamma, \Theta))$, using the fact that $\mathcal{K}_{P}^{s, \gamma}\left(X^{\wedge}\right)=\varliminf_{j \in \mathbb{N}} E^{j}$ is a Fréchet space with group action for an appropriate choice of spaces $E^{j}$, cf. similarly, formula (1.1.12).

Remark 2.3.1. Setting $\mathbb{W}_{0}:=\mathbb{R}_{+} \times Y \times\left(\overline{\mathbb{R}}_{+} \times X\right)$ as the stretched manifold to $W_{0}=\mathbb{R}_{+} \times Y \times X^{\Delta}$ which is a manifold with edge $\mathbb{R}_{+} \times Y$ and model cone $X^{\Delta}$, we have

$$
\begin{aligned}
& \mathcal{V}^{s, \delta}\left(\mathbb{R}_{+} \times Y, \mathcal{K}^{s, \gamma}\left(X^{\wedge}\right)\right) \subset \mathcal{W}_{\text {loc }}^{s, \gamma}\left(\mathbb{W}_{0}\right), \\
& \mathcal{V}^{s, \delta}\left(\mathbb{R}_{+} \times Y, \mathcal{K}_{P}^{s, \gamma}\left(X^{\wedge}\right)\right) \subset \mathcal{W}_{P, \text { loc }}^{s, \gamma}\left(\mathbb{W}_{0}\right)
\end{aligned}
$$

for every $s, \gamma, \delta \in \mathbb{R}$ and any asymptotic type $P \in \operatorname{As}(X,(\gamma, \Theta))$.

This allows us to define global corner spaces on $\mathbb{R}_{+} \times W$ for an arbitrary compact manifold $W$ with edges. As usual, everything will be done for the corresponding stretched manifold $\mathbb{W}$. Let $M$ denote the double of $\mathbb{W}$ which is a closed $C^{\infty}$ manifold of dimension $m=1+n+q$ when the edge $Y$ is of dimension $q$ and the base $X$ of the model cone of dimension $n$. We then have $M=\mathbb{W}_{-} \cup \mathbb{W}_{+}$and $\partial \mathbb{W}=\mathbb{W}_{-} \cap \mathbb{W}_{+}$, where $\mathbb{W}_{-}$and $\mathbb{W}_{+}$are copies of $\mathbb{W}$ that are glued together along $\partial \mathbb{W}$.

In Section 1.1 we have introduced the spaces $\mathcal{H}^{s, \delta}\left(M^{\wedge}\right)$. We now choose any $\chi \in C^{\infty}(M)$ such that supp $\chi \subset \operatorname{int} \mathbb{W}$ (where $\mathbb{W}$ is identified with $\mathbb{W}_{+}$) and 
(in our fixed global splitting of variables near $\partial \mathbb{W}$ into $(r, x, y) \in[0,1) \times X \times Y$ ) $\chi=0$ for $0 \leq r \leq 1 / 2, \chi=1$ on $\mathbb{W} \backslash([0,(3 / 4)) \times X \times Y)$. Then, for any $\varphi(r) \in C_{0}^{\infty}(0,1)$ we have

$$
\varphi \mathcal{V}^{s, \delta}\left(\mathbb{R}_{+} \times Y, \mathcal{K}^{s, \gamma}\left(X^{\wedge}\right)\right) \subset \mathcal{H}^{s, \delta}\left(M^{\wedge}\right) .
$$

We now introduce the spaces

$$
\mathcal{V}^{s,(\gamma, \delta)}\left(\mathbb{W}^{\wedge}\right):=\left\{\omega v+\chi h: v \in \mathcal{V}^{s, \delta}\left(\mathbb{R}_{+} \times Y, \mathcal{K}^{s, \gamma}\left(X^{\wedge}\right)\right), h \in \mathcal{H}^{s, \delta}\left(M^{\wedge}\right)\right\}
$$

where $\omega:=1-\chi$, and analogously

$$
\mathcal{V}_{P}^{s,(\gamma, \delta)}\left(\mathbb{W}^{\wedge}\right):=\left\{\omega v+\chi h: v \in \mathcal{V}^{s, \delta}\left(\mathbb{R}_{+} \times Y, \mathcal{K}_{P}^{s, \gamma}\left(X^{\wedge}\right)\right), h \in \mathcal{H}^{s, \delta}\left(M^{\wedge}\right)\right\} .
$$

By virtue of relation (2.3.2) this is a correct definition, i.e., independent of the choice of $\chi$.

In (2.3.3) we can choose Hilbert space structures that define norms in the spaces, and (2.3.4) is a Fréchet space in a natural way.

Notice that the weight $\delta$ in (2.3.3) and (2.3.4) concerns $t \rightarrow 0$ as well as $t \rightarrow \infty$ (similarly to the meaning of the weight in the spaces $\mathcal{H}^{s, \delta}\left(M^{\wedge}\right)$ ); for instance, we have

$$
t^{\beta} \mathcal{V}_{P}^{s,(\gamma, \delta)}\left(\mathbb{W}^{\wedge}\right)=\mathcal{V}_{P}^{s,(\gamma, \delta+\beta)}\left(\mathbb{W}^{\wedge}\right)
$$

for arbitrary $s, \gamma, \delta, \beta$ (and a similar relation for the spaces without subscript $P)$. Here, we are mainly interested in the behaviour for $t \rightarrow 0$.

\section{$\S 2.4$. Iterated asymptotics}

Our text goal is to introduce iterated asymptotics of weighted Sobolev distributions on a manifold $M$ with edges and corners, where neighbourhoods of corners are modelled by cones $W^{\Delta}$ for a compact manifold $W$ with edges. If $M^{\prime \prime}=\left\{c_{1}, \ldots, c_{N}\right\}$ denotes the finite set of corner points, $M \backslash M^{\prime \prime}$ is a manifold with smooth edges; corresponding asymptotics near the edges have been introduced in Section 1.4. Thus, it suffices to mainly look at $W^{\Delta}$, in fact, at the stretched cone $\mathbb{W}^{\wedge} \ni(t, \widetilde{x})$, and asymptotics for $t \rightarrow 0$.

Fix a weight interval $\Xi=(\xi, 0],-\infty \leq \xi<0$, and set

$$
\mathcal{V}_{P, \Xi}^{s,(\gamma, \delta)}\left(\mathbb{W}^{\wedge}\right)={\underset{\varepsilon}{\check{\varepsilon}>0}}_{\lim ^{-\xi-\varepsilon}} \mathcal{V}_{P}^{s,(\gamma, \delta)}\left(\mathbb{W}^{\wedge}\right),
$$


$P \in \operatorname{As}(X,(\gamma, \Theta))$. Here, $k \in C^{\infty}\left(\mathbb{R}_{+}\right)$is any strictly positive function such that $k(t)=t$ for $0<t<c_{0}$ and $k(t)=1$ for $c_{1}<t \leq \infty$ for certain constants $c_{0}<c_{1}$. The space (2.4.1) is Fréchet in a natural way (and, of course, independent of the choice of the function $k)$. For $\Xi_{i}=\left(\xi_{i}, 0\right], i=1,2$, and $\xi_{2} \leq \xi_{1}<0$, we have continuous embeddings

$$
\mathcal{V}_{P, \Xi_{2}}^{s,(\gamma, \delta)}\left(\mathbb{W}^{\wedge}\right) \hookrightarrow \mathcal{V}_{P, \Xi_{1}}^{s,(\gamma, \delta)}\left(\mathbb{W}^{\wedge}\right)
$$

for all $s, \gamma, \delta \in \mathbb{R}$.

Let us fix weight data

$$
\boldsymbol{h}=(\delta, \Xi), \quad \Xi=(\xi, 0], \quad-\infty \leq \xi<0,
$$

and an asymptotic type $P \in \operatorname{As}(X, \boldsymbol{g})$ for $\boldsymbol{g}=(\gamma, \Theta)$. Let $\operatorname{As}\left(\mathbb{W}, \boldsymbol{h}^{\bullet}\right)_{P}$ denote the set of all discrete asymptotic types

$$
Q=\left\{\left(q_{j}, n_{j}, L_{j}\right)\right\}_{0 \leq j \leq N},
$$

where $N=N(Q) \leq \infty$ and $N(Q)<\infty$ for finite $\Xi$, defined by the following properties:

$$
\pi_{\mathbb{C}} Q:=\left\{q_{j}\right\}_{0 \leq j \leq N} \subset S_{\left(\frac{m+1}{2}-\delta+\xi, \frac{m+1}{2}-\delta\right)}
$$

for $m=\operatorname{dim} \mathbb{W}, \operatorname{Re} q_{j} \rightarrow-\infty$ as $j \rightarrow \infty$ for $N(Q)=\infty, n_{j} \in \mathbb{N}$, and $L_{j} \subset$ $\mathcal{W}_{P}^{\infty, \gamma}(\mathbb{W})$ is a finite-dimensional subspace for all $j$, where $P$ is independent of $j$.

Definition 2.4.1. $\quad$ Let $P \in \operatorname{As}(X, \boldsymbol{g})$ for $\boldsymbol{g}=(\gamma, \Theta)$, and $Q \in \operatorname{As}(\mathbb{W}$, $\left.\boldsymbol{h}^{\bullet}\right)_{P}$ for $\boldsymbol{h}=(\delta, \Xi)$. We then define $\mathcal{V}_{P, Q}^{s,(\gamma, \delta)}\left(\mathbb{W}^{\wedge}\right)$ to be the subspace of all $u \in$ $\mathcal{V}_{P}^{s,(\gamma, \delta)}\left(\mathbb{W}^{\wedge}\right)$ such that there are elements $c_{j k} \in L_{j}, 0 \leq k \leq n_{j}, 0 \leq j \leq N(Q)$, where for every $\xi<\beta<0$ there is an $M_{\beta}$ such that

$$
u(t, \widetilde{x})-\omega(t) \sum_{j=0}^{M} \sum_{k=0}^{n_{j}} c_{j k}(\widetilde{x}) t^{-q_{j}} \log ^{k} t \in \mathcal{V}_{P,(\beta, 0]}^{s,(\gamma, \delta)}\left(\mathbb{W}^{\wedge}\right)
$$

for all $M \geq M_{\beta}$ and any cut-off function $\omega(t)$.

The coefficients $c_{j k}$ are uniquely determined by $u$. Let, in particular, $\Xi$ be finite. Then, in condition (2.4.2) we may set $\beta=\xi$ and $M=N(Q)$. In this case let $\mathcal{E}_{Q}\left(\mathbb{W}^{\wedge}\right)$ denote the linear span of functions $\omega(t) c_{j k}(\widetilde{x}) t^{-q_{j}} \log ^{k} t$ for all $c_{j k} \in L_{j}, 0 \leq k \leq n_{j}, 0 \leq j \leq N(Q)$, with some fixed cut-off function $\omega(t)$. We then have

$$
\mathcal{V}_{P, Q}^{s,(\gamma, \delta)}\left(\mathbb{W}^{\wedge}\right)=\mathcal{V}_{P, \Xi}^{s,(\gamma, \delta)}\left(\mathbb{W}^{\wedge}\right)+\mathcal{E}_{Q}\left(\mathbb{W}^{\wedge}\right)
$$


which is a direct decomposition. The space $\mathcal{V}_{P, \Xi}^{s,(\gamma, \delta)}\left(\mathbb{W}^{\wedge}\right)$ is Fréchet, and $\mathcal{E}_{Q}\left(\mathbb{W}^{\wedge}\right)$ is of finite dimension. This gives us a Fréchet topology in the space $\mathcal{V}_{P, Q}^{s,(\gamma, \delta)}\left(\mathbb{W}^{\wedge}\right)$ (that is independent of the choice of $\omega$ ). For infinite $\Xi$ we can choose a sequence $\left(\xi_{k}\right)_{k \in \mathbb{N}}$ of reals with $\xi_{k+1}<\xi_{k}<0$ for all $k$ and $\xi_{k} \rightarrow-\infty$ as $k \rightarrow \infty$, and form $Q_{k}=\left\{(q, n, L) \in Q: \operatorname{Re} q>(m+1) / 2-\delta+\xi_{k}\right\}, k \in \mathbb{N}$. We then have continuous embeddings $\mathcal{V}_{P, Q_{k+1}}^{s,(\gamma, \delta)}\left(\mathbb{W}^{\wedge}\right) \hookrightarrow \mathcal{V}_{P, Q_{k}}^{s,(\gamma, \delta)}\left(\mathbb{W}^{\wedge}\right)$ for all $k$, and we get

$$
\mathcal{V}_{P, Q}^{s,(\gamma, \delta)}\left(\mathbb{W}^{\wedge}\right)=\varliminf_{k \in \mathbb{N}} \mathcal{l i m}_{P, Q_{k}}^{s,(\gamma, \delta)}\left(\mathbb{W}^{\wedge}\right)
$$

with the Fréchet topology of the projective limit.

Remark 2.4.2. Let $\varphi(t) \in C^{\infty}\left(\overline{\mathbb{R}}_{+}\right)$and assume that either $\varphi(t) \in$ $C_{0}^{\infty}\left(\overline{\mathbb{R}}_{+}\right)$or $\varphi(t)=1-\psi(t)$ for some $\psi(t) \in C_{0}^{\infty}\left(\overline{\mathbb{R}}_{+}\right)$. Let $\mathcal{H}$ denote one of the spaces $\mathcal{V}^{s,(\gamma, \delta)}\left(\mathbb{W}^{\wedge}\right), \mathcal{V}_{P}^{s,(\gamma, \delta)}\left(\mathbb{W}^{\wedge}\right)$, or $\mathcal{V}_{P, Q}^{s,(\gamma, \delta)}\left(\mathbb{W}^{\wedge}\right)$ for certain $P \in \operatorname{As}(X, \boldsymbol{g})$, $Q \in \operatorname{As}\left(\mathbb{W}, \boldsymbol{h}^{\bullet}\right)_{P}$. Then $u \in \mathcal{H}$ implies $\varphi u \in \mathcal{H}$.

\section{§3. The Edge-Corner Algebra with Trace and Potential Conditions}

\section{$\S 3.1$. Green corner operators}

In this paper a manifold $M$ with edges and corners is a topological space (paracompact and locally compact) with subspaces $M^{\prime \prime} \subset M^{\prime} \subset M$, where $M^{\prime \prime}$ is the finite set of corner points, $M \backslash M^{\prime}$ and $M^{\prime} \backslash M^{\prime \prime}$ are $C^{\infty}$ manifolds, and $M \backslash M^{\prime \prime}$ is a manifold with edges. In addition, we assume that every $c \in M^{\prime}$ has a neighbourhood $V$ that is homeomorphic to $([0, \varepsilon) \times W) /(\{0\} \times W)$ for some $\varepsilon>0$, where $W$ is a compact manifold with edges $Y$, and that there is fixed a corner structure on $V$ in terms of a splitting of variables on $[0, \varepsilon) \times \mathbb{W}$ into $(t, \widetilde{x})$, interpreted as a restriction of variables from $\mathbb{R} \times \mathbb{W}$ to $[0, \varepsilon) \times \mathbb{W}$.

We have a notion of equivalence of corner structures by saying that another splitting $\left(t^{\prime}, \widetilde{x}^{\prime}\right)$ is equivalent to $(t, \widetilde{x})$ if $(t, \widetilde{x}) \rightarrow\left(t^{\prime}, \widetilde{x}^{\prime}\right)$ comes from a restriction of a diffeomorphism $\mathbb{R} \times \mathbb{W} \rightarrow \mathbb{R} \times \mathbb{W}$ in the sense of $C^{\infty}$ manifolds with boundary, where in addition the $X$-bundles (that constitute the boundary) are isomorphically transformed to each other.

For simplicity, we shall keep the corner axis $t \in \mathbb{R}_{+}$fixed in a neighbourhood of zero. Moreover, transition maps "in $\mathbb{W}$-direction" near $t=0$ are assumed to preserve the chosen global splittings of variables $(r, x, y)$ in a neighbourhood of $\partial \mathbb{W}$. Clearly, these conditions could be considerably weakend, but the analysis of operators with asymptotics requires a choice of an atlas with 
some additional structure, though we do not lose generality in the sense that such an atlas may always be found (recall that $\partial \mathbb{W}$ was assumed to be a trivial $X$-bundle on $Y$, cf. Section 1.4). With $M$ we associate a stretched manifold $\mathbb{M}$, locally modelled by $\overline{\mathbb{R}}_{+} \times \mathbb{W}$ near stretched corner points $\{0\} \times \mathbb{W}$ (cf. the notation in the introduction).

By construction, on $\mathbb{M}$ we have locally near $\mathbb{M}_{\text {sing }}:=\{0\} \times \partial \mathbb{W}$ the variables $(t, r, x, y)$, where $t \in \overline{\mathbb{R}}_{+}$is the corner axis variable, $r \in \mathbb{R}_{+}$the cone axis variable from the model cone of $\mathbb{W}$ near $\partial \mathbb{W}$, and $x \in X, y \in Y$. Recall that $X$ is the base of the model cone and $Y$ the edge of $W$.

To simplify notation we assume that $M^{\prime \prime}$ consists of one corner point $v$. (We admit the base $W$ of the corner to have different connected components; so the general case is covered anyway). In addition, to avoid "comp"- and "loc"-subscripits in Sobolev spaces we assume $M$ to be compact; then also $\mathbb{M}$ is compact.

Given fixed Riemannian metrics on $X$ and $Y$, respectively, we construct a Riemannian metric on $\mathbb{M}$ by taking the product metric from $\overline{\mathbb{R}}_{+} \times \overline{\mathbb{R}}_{+} \times X \times Y$ near $\{0\} \times \partial \mathbb{W}$ and gluing it together with a metric on $\mathbb{M}_{\text {reg }}:=\mathbb{M} \backslash(\{0\} \times \mathbb{W})$ in the sense of a stretched manifold with edge.

Vector bundles $E$ on $\mathbb{M}$ are assumed to be equipped with Hermitian metrics with an analogous product structure with respect to variables on $\mathbb{M}$ near $\{0\} \times$ $\partial \mathbb{W}$. (Concerning $\left.E\right|_{\mathbb{M}_{\text {reg }}}$ we impose the same conditions as above). We then have the spaces $L^{2}(\mathbb{M}, E)$ with given scalar products.

On $\mathbb{M}$ we have singular charts of different kind, namely

$$
\chi_{\text {corner }}: \mathbb{V} \rightarrow \overline{\mathbb{R}}_{+} \times \overline{\mathbb{R}}_{+} \times \Sigma \times \Omega \ni(t, r, x, y)
$$

for open $\Sigma \subseteq \mathbb{R}^{n}, \Omega \subseteq \mathbb{R}^{q}$, with (stretched) corner neighbourhoods $\mathbb{V}$ on $\mathbb{M}$ (where $\mathbb{V} \cap \mathbb{M}_{\text {sing }} \neq \emptyset$ ), moreover,

$$
\chi_{\text {cone }}: \mathbb{K} \rightarrow \overline{\mathbb{R}}_{+} \times \Delta \ni(t, \widetilde{x})
$$

for open $\Delta \subseteq \mathbb{R}^{1+n+q}$, with (stretched) cone neighbourhoods $\mathbb{K}$ on $\mathbb{M}$ (where $\mathbb{K} \cap \mathbb{M}_{\text {sing }} \neq \emptyset$ ), and

$$
\chi_{\text {edge }}: \mathbb{L} \rightarrow \overline{\mathbb{R}}_{+} \times \Psi \ni(r, \widetilde{y})
$$

for open $\Psi \subseteq \mathbb{R}^{1+n+q}$, with (stretched) wedge neighbourhoods $\mathbb{L}$ on $\mathbb{M}$ (where $\mathbb{L} \cap \mathbb{M}_{\text {sing }}=\emptyset, \mathbb{L} \cap \partial \mathbb{M}_{\text {reg }} \neq \emptyset$; the latter notation treats $\mathbb{M}_{\text {reg }}$ as a stretched manifold with edges). Finally, there are the standard (regular) charts on the $C^{\infty}$ manifold $M \backslash M^{\prime}$, namely

$$
\chi_{\mathrm{int}}: U \rightarrow \Gamma \ni m
$$


for coordinate neighbourhoods $U \subset M \backslash M^{\prime}$ and open sets $\Gamma \subseteq \mathbb{R}^{2+n+q}$.

Notation from Sections 2.3 and 2.4 concerning weighted spaces on $\mathbb{W}^{\wedge}$ (with and without asymptotics) directly generalise to the case of distributional sections of vector bundles $E$. In other words, we have spaces

$$
\mathcal{V}^{s,(\gamma, \delta)}\left(\mathbb{W}^{\wedge}, E\right), \quad \mathcal{V}_{P}^{s,(\gamma, \delta)}\left(\mathbb{W}^{\wedge}, E\right), \quad \mathcal{V}_{P, Q}^{s,(\gamma, \delta)}\left(\mathbb{W}^{\wedge}, E\right)
$$

for $P \in \operatorname{As}(X, \boldsymbol{g}), Q \in \operatorname{As}\left(\mathbb{W}, h^{\bullet}\right)_{P}$ (coefficients of asymptotics are, of course, related to $E$ in an evident manner). In the following definition the latter $E$ is used for a bundle on $\mathbb{M}$ as well as for its restriction to a neighbourhood of $\mathbb{M}_{\text {sing }}$ that is also regarded as a restriction of a bundle on $\mathbb{W}^{\wedge}$, again denoted by $E$ (we hope, this will not cause confusion). Moreover, we fix a cut-off function $\omega(t)$ supported in a small neighbourhood of $t=0$.

Definition 3.1.1. We set

$$
\begin{aligned}
& \mathcal{H}^{s,(\gamma, \delta)}(\mathbb{M}, E):=[\omega] \mathcal{V}^{s,(\gamma, \delta)}\left(\mathbb{W}^{\wedge}, E\right)+[1-\omega] \mathcal{W}^{s, \gamma}\left(\mathbb{M}_{\text {reg }}, E\right), \\
& \mathcal{H}_{P}^{s,(\gamma, \delta)}(\mathbb{M}, E):=[\omega] \mathcal{V}_{P}^{s,(\gamma, \delta)}\left(\mathbb{W}^{\wedge}, E\right)+[1-\omega] \mathcal{W}_{P, \text { loc }}^{s, \gamma}\left(\mathbb{M}_{\text {reg }}, E\right), \\
& \mathcal{H}_{P, Q}^{s,(\gamma, \delta)}(\mathbb{M}, E):=[\omega] \mathcal{V}_{P, Q}^{s,(\gamma, \delta)}\left(\mathbb{W}^{\wedge}, E\right)+[1-\omega] \mathcal{W}_{P, \text { loc }}^{s, \gamma}\left(\mathbb{M}_{\text {reg }}, E\right),
\end{aligned}
$$

for $P \in \operatorname{As}(X, \boldsymbol{g}), Q \in \operatorname{As}\left(\mathbb{W}, \boldsymbol{h}^{\bullet}\right)_{P}, s, \gamma, \delta \in \mathbb{R}$.

This is a correct definition in the sense that the spaces are independent of the choice of $\omega$. In (3.1.5) we get Hilbert space structures for fixed $\omega$, while (3.1.6) and (3.1.7) are Fréchet spaces in the non-direct sum topology.

For purposes below, for given $\alpha, \beta \in \mathbb{R}$ we fix a strictly positive function $l^{\alpha, \beta}$ on $\mathbb{M}_{\text {reg }} \backslash \partial \mathbb{M}_{\text {reg }}$ that equals

$$
l^{\alpha, \beta}=\left\{\omega(t) t^{\alpha}+(1-\omega(t))\right\} r^{\beta}
$$

near $\mathbb{M}_{\text {sing }}$, where $\omega(t)$ is a cut-off function that vanishes for $t>\varepsilon / 2$ (the variable $t$ refers to $(0, \varepsilon) \times \mathbb{W}$, while $r$ is the cone axis variable for the local wedge of $\mathbb{M}_{\text {reg }}$ near $\partial \mathbb{M}_{\text {reg }}$ ), and $l^{\alpha, \beta}$ equals $r^{\beta}$ near $\partial \mathbb{M}_{\text {reg }}$ outside an $\varepsilon / 2-$ neighbourhood of $\mathbb{M}_{\text {sing }}$ with respect to $t$. Set for a moment $l(n, q):=l^{-(1+n+q) / 2,-n / 2}$. We then have

$$
\mathcal{H}^{0,(0,0)}(\mathbb{M}, E)=l(n, q) L^{2}(\mathbb{M}, E),
$$

and we endow $\mathcal{H}^{0,(0,0)}(\mathbb{M}, E)$ with the scalar product

$$
(f, g)=\left(l(n, q)^{-1} f, l(n, q)^{-1} g\right)_{L^{2}(\mathbb{M}, E)}
$$


for $f, g \in \mathcal{H}^{0,(0,0)}(\mathbb{M}, E)$. Taking this as a reference scalar product for the scale $\mathcal{H}^{s,(\gamma, \delta)}(\mathbb{M}, E)$, there is a non-degenerate sesquilinear pairing

$$
\mathcal{H}^{s,(\gamma, \delta)}(\mathbb{M}, E) \times \mathcal{H}^{-s,(-\gamma,-\delta)}(\mathbb{M}, E) \rightarrow \mathbb{C}
$$

induced by $C_{0}^{\infty}\left(\mathbb{M}_{\text {reg }} \backslash \partial \mathbb{M}_{\text {reg }}\right) \times C_{0}^{\infty}\left(\mathbb{M}_{\text {reg }} \backslash \partial \mathbb{M}_{\text {reg }}\right) \rightarrow \mathbb{C}$ via $(\cdot, \cdot)$.

Let us now set for abbreviation $B:=M^{\prime}$ which is a compact manifold with conical singularities $M^{\prime \prime}$ (that is a single point in our simplified model). Then, we have weighted Sobolev spaces $\mathcal{H}^{s, \beta}(\mathbb{B})$ for $s, \beta \in \mathbb{R}$; here, $\mathbb{B}$ is the stretched manifold associated with $B$. Recall that when $Y$ denotes the base of the conical singularity $c$ in $B$, i.e., $B$ is locally near $c$ modelled by $Y^{\Delta}$, we have $\mathcal{H}^{s, \beta}(\mathbb{B})=[\omega] \mathcal{H}^{s, \beta}\left(Y^{\wedge}\right)+[1-\omega] H_{\text {loc }}^{s}(\operatorname{int} \mathbb{B})$ for a cut-off function $\omega(t)$; the spaces $\mathcal{H}^{s, \beta}\left(Y^{\wedge}\right)$ have been defined in Section 1.1 above. Analogously, we define weighted spaces $\mathcal{H}^{s, \beta}(\mathbb{B}, J)$ of distributional sections in vector bundles $J$ on $\mathbb{B}$. Concerning dimensions, for the corner base $W$ we employ the notation from Section 1.1, in particular, $q=\operatorname{dim} Y$ with $Y$ being the edge of $W, n=\operatorname{dim} X$, where $X$ is the base of the model cone for $W$. Then the edges of $M \backslash M^{\prime \prime}$ are of dimension $q+1$, i.e., $\operatorname{dim} \mathbb{B}=q+1$, and $\operatorname{dim} \mathbb{M}_{\text {reg }}=2+n+q$. Thus, the edge of $M \backslash M^{\prime \prime}$ is of codimension $n+1$ in $M \backslash M^{\prime \prime}$, and hence it is adequate to look at direct sums of spaces

$$
\mathcal{H}^{s,(\gamma, \delta)}(\mathbb{M} ; \boldsymbol{m}):=\mathcal{H}^{s,(\gamma, \delta)}(\mathbb{M}, E) \oplus \mathcal{H}^{s-\frac{n+1}{2}, \delta-\frac{n+1}{2}}(\mathbb{B}, J)
$$

for vector bundles $E$ and $J$ on $\mathbb{M}$ and $\mathbb{B}$, respectively, where $\boldsymbol{m}:=(E, J)$.

To have a short description for anti-duality with respect to pairings induced by $(\cdot, \cdot)_{\mathcal{H}^{0,(0,0)}(\mathbb{M}, E)}$ and $(\cdot, \cdot)_{\mathcal{H}^{0,0}(\mathbb{B}, J)}$, respectively, we also set

$$
\mathcal{H}^{s^{*},\left(\gamma, \delta^{*}\right)}(\mathbb{M} ; \boldsymbol{m}):=\mathcal{H}^{s,(\gamma, \delta)}(\mathbb{M}, E) \oplus \mathcal{H}^{s+\frac{n+1}{2}, \delta+\frac{n+1}{2}}(\mathbb{B}, J) .
$$

Analogously, we set

$$
\mathcal{H}_{P}^{s,(\gamma, \delta)}(\mathbb{M} ; \boldsymbol{m}):=\mathcal{H}_{P_{1}, P_{2}}^{s,(\gamma, \delta)}(\mathbb{M}, E) \oplus \mathcal{H}_{P_{3}}^{s-\frac{n+1}{2}, \delta-\frac{n+1}{2}}(\mathbb{B}, J)
$$

for $P:=\left(P_{1}, P_{2}, P_{3}\right)$, where $P_{1} \in \operatorname{As}(X, \boldsymbol{g}), P_{2} \in \operatorname{As}\left(\mathbb{W}, h^{\bullet}\right)_{P_{1}}, P_{3} \in \operatorname{As}\left(Z, l^{\bullet}\right)$ for $\boldsymbol{g}=(\gamma, \Theta), \boldsymbol{h}=(\delta, \Xi), \boldsymbol{l}=(\delta-(n+1) / 2, \Xi)$ (and, similarly, with $*$ at $s$ and $\delta)$.

Let $\operatorname{As}\left(X, \mathbb{W}, Z ; \boldsymbol{g}, \boldsymbol{h}^{\bullet}, \boldsymbol{l}^{\bullet}\right)$ denote the set of all such triples $P=\left(P_{1}, P_{2}, P_{3}\right)$.

Proposition 3.1.2. There are canonical continuous embeddings

$$
\mathcal{H}^{s^{\prime},\left(\gamma^{\prime}, \delta^{\prime}\right)}(\mathbb{M}, E) \hookrightarrow \mathcal{H}^{s,(\gamma, \delta)}(\mathbb{M}, E)
$$


for $s^{\prime} \geq s, \gamma^{\prime} \geq \gamma, \delta^{\prime} \geq \delta$ that are compact for $s^{\prime}>s, \gamma^{\prime}>\gamma, \delta^{\prime}>\delta$. Moreover, we have continuous embeddings

$$
\mathcal{H}_{P}^{s^{\prime},(\gamma, \delta)}(\mathbb{M}, E) \hookrightarrow \mathcal{H}_{P}^{s,(\gamma, \delta)}(\mathbb{M}, E), \quad \mathcal{H}_{P, Q}^{s^{\prime},(\gamma, \delta)}(\mathbb{M}, E) \hookrightarrow \mathcal{H}_{P, Q}^{s,(\gamma, \delta)}(\mathbb{M}, E)
$$

(in the notation of Definition 3.1.1) for $s^{\prime} \geq s$.

The proof of Proposition 3.1.2 is not really difficult and left to the reader. In a similar (but simpler) situation assertions of this type for weighted cone and edge Sobolev spaces may be found in [29, Theorems 2.1.53, 3.1.23].

Remark 3.1.3. For every $P \in \operatorname{As}(X, \boldsymbol{g}), \boldsymbol{g}=(\gamma, \Theta)$, and $Q \in \operatorname{As}(\mathbb{W}, \boldsymbol{h})_{P}$, $\boldsymbol{h}=(\delta, \Xi)$, there is an $\varepsilon>0$ such that

$$
\mathcal{H}_{P, Q}^{s,(\gamma, \delta)}(\mathbb{M}, E) \hookrightarrow \mathcal{H}^{s,(\gamma+\varepsilon, \delta+\varepsilon)}(\mathbb{M}, E)
$$

for all $s \in \mathbb{R}$.

The observation of Remark 3.1.3 follows from $\operatorname{dist}\left(\pi_{\mathbb{C}} Q, \Gamma_{(m+1) / 2-\delta}\right)>\varepsilon$ and $\operatorname{dist}\left(\pi_{\mathbb{C}} P, \Gamma_{(n+1) / 2-\gamma}\right)>\varepsilon$ for some $\varepsilon>0$. Then we have, for instance, $\mathcal{K}_{P}^{s, \gamma}\left(X^{\wedge}\right) \hookrightarrow \mathcal{K}^{s, \gamma+\varepsilon}\left(X^{\wedge}\right)$ which entails $\mathcal{V}_{P}^{s,(\gamma, \delta)}\left(\mathbb{W}^{\wedge}\right) \hookrightarrow \mathcal{V}^{s,(\gamma+\varepsilon, \delta)}\left(\mathbb{W}^{\wedge}\right)$, cf. (2.3.4). The improvement of $\delta$ by $\varepsilon$ is obvious anyway.

Definition 3.1.4. Let $\boldsymbol{g}=(\gamma, \varrho, \Theta ; \delta, \sigma, \Xi)$ and $\boldsymbol{v}=\left(E, F ; J_{-}, J_{+}\right)$ (where $E, F$ are vector bundles on $\mathbb{B}$ ). Then $\mathcal{C}_{G}(\mathbb{M}, \boldsymbol{g} ; \boldsymbol{v})$ is defined to be the space of all operators

$$
G: \mathcal{H}^{s,(\gamma, \delta)}(\mathbb{M} ; \boldsymbol{m}) \rightarrow \mathcal{H}^{\infty,(\varrho, \sigma)}(\mathbb{M} ; \boldsymbol{n})
$$

continuous for all $s \in \mathbb{R}$, such that there are triples of asymptotic types $P \in$ $\operatorname{As}\left(X, \mathbb{W}, Z ;(\varrho, \Theta),(\sigma, \Xi)^{\bullet},(\sigma-(n+1) / 2, \Xi)^{\bullet}\right)$ and $Q \in \operatorname{As}(X, \mathbb{W}, Z ;(-\gamma, \Theta)$, $\left.(-\delta, \Xi)^{\bullet},(-\delta-(n+1) / 2, \Xi)^{\bullet}\right)$ such that $G$ induces continuous operators

$$
G: \mathcal{H}^{s,(\gamma, \delta)}(\mathbb{M} ; \boldsymbol{m}) \rightarrow \mathcal{H}_{P}^{\infty,(\varrho, \sigma)}(\mathbb{M} ; \boldsymbol{n})
$$

and

$$
G^{*}: \mathcal{H}^{s^{*},\left(-\varrho,(-\sigma)^{*}\right)}(\mathbb{M} ; \boldsymbol{n}) \rightarrow \mathcal{H}_{Q}^{\infty,\left(-\gamma,(-\delta)^{*}\right)}(\mathbb{M} ; \boldsymbol{m})
$$

for all $s \in \mathbb{R} ;$ here, $\boldsymbol{m}:=\left(E, J_{-}\right), \boldsymbol{n}:=\left(F, J_{+}\right)$. The elements of $\mathcal{C}_{G}(\mathbb{M}, \boldsymbol{g} ; \boldsymbol{v})$ are called Green corner operators. 
In the sequel we specify the choice of weight intervals by setting $\Theta:=$ $(-(k+1), 0]$ for $-\infty \leq k<0$ and $\Xi:=(-(l+1), 0]$ for $-\infty<l<0$ (later on, we also admit $l=-\infty)$.

In the following we assume that a neighbourhood of $\mathbb{M}_{\text {sing }}$ in $\mathbb{M}$ is identified with $[0,1) \times \mathbb{W}$; then functions $\varphi \in C_{0}^{\infty}\left(\overline{\mathbb{R}}_{+}\right)$that are supported in $[0,1)$ will also be interpreted as functions on $\mathbb{M}$.

Given an element $f(t, w) \in C^{\infty}\left(\overline{\mathbb{R}}_{+}, \mathcal{M}_{R}^{-\infty}(\mathbb{W}, \boldsymbol{g} ; \boldsymbol{v})\right), R:=\left(R_{1}, R_{2}\right) \in$ $\mathbf{A s}_{M+G}(X, \boldsymbol{g} ; \boldsymbol{v}) \times \mathbf{A} \mathbf{s}^{\bullet}(\mathbb{W} ; \boldsymbol{v})_{R_{1}}$, where $\boldsymbol{g}=(\gamma, \gamma-\mu, \Theta), \boldsymbol{v}=\left(E, F ; J_{-}, J_{+}\right)$, cf. Definition 2.2.2, we can form operators

$$
\varphi(t) \operatorname{op}_{M}^{\delta-\frac{m}{2}}(f) \widetilde{\varphi}(t): \mathcal{H}^{s,(\gamma, \delta)}(\mathbb{M} ; \boldsymbol{m}) \rightarrow \mathcal{H}^{\infty,(\gamma-\mu, \delta)}(\mathbb{M} ; \boldsymbol{n})
$$

for $m:=1+n+q, \boldsymbol{m}=\left(E, J_{-}\right), \boldsymbol{n}=\left(F, J_{+}\right)$, and $\varphi, \widetilde{\varphi} \in C_{0}^{\infty}\left(\overline{\mathbb{R}}_{+}\right)$supported in $[0,1)$. It can easily be proved that for every

$$
P=\left(P_{1}, P_{2}, P_{3}\right) \in \operatorname{As}\left(X, \mathbb{W}, Z ; \boldsymbol{g}, \boldsymbol{h}^{\bullet}, \boldsymbol{l}^{\bullet}\right)
$$

for $\boldsymbol{g}=(\gamma, \Theta), \boldsymbol{h}=(\delta, \Xi), \boldsymbol{l}=(\delta-(n+1) / 2, \Xi)$, there exists a

$$
Q=\left(Q_{1}, Q_{2}, Q_{3}\right) \in \operatorname{As}\left(X, \mathbb{W}, Z ; \widetilde{\boldsymbol{g}}, h^{\bullet}, l^{\bullet}\right)
$$

for $\widetilde{\boldsymbol{g}}=(\gamma-\mu, \Theta)$, such that

$$
\varphi(t) \operatorname{op}_{M}^{\delta-\frac{m}{2}}(f) \widetilde{\varphi}(t): \mathcal{H}_{P}^{s,(\gamma, \delta)}(\mathbb{M} ; \boldsymbol{m}) \rightarrow \mathcal{H}_{Q}^{\infty,(\gamma-\mu, \delta)}(\mathbb{M} ; \boldsymbol{n})
$$

is a continuous operator for all $s \in \mathbb{R}$.

Remark 3.1.5. Let $\varphi, \widetilde{\varphi} \in C_{0}^{\infty}([0,1))$ and assume that $\varphi=0$ or $\widetilde{\varphi}=0$ in a neighbourhood of $t=0$. Then we have

$$
\varphi \operatorname{op}_{M}^{\delta-\frac{m}{2}}(f) \widetilde{\varphi} \in \mathcal{C}_{G}(\mathbb{M}, \boldsymbol{k} ; \boldsymbol{v})
$$

for $\boldsymbol{k}=(\gamma, \gamma-\mu, \Theta ; \delta, \delta, \Xi)$. Moreover, if $\varphi, \widetilde{\varphi} \in C_{0}^{\infty}([0,1))$ are arbitrary, we have

$$
\varphi t^{\beta} \operatorname{op}_{M}^{\delta-\frac{m}{2}}(f) \widetilde{\varphi}, \varphi \operatorname{op}_{M}^{\delta-\frac{m}{2}}(f) t^{\beta} \widetilde{\varphi} \in \mathcal{C}_{G}(\mathbb{M}, \boldsymbol{k} ; \boldsymbol{v})
$$

for every $\beta \geq l+1$ (recall that $\Xi=(-(l+1), 0])$ ).

These relations can be verified in a similar manner as the corresponding ones in the simpler situation for conical singularities, cf. [26, Section 2.1.5], see also [29, Section 2.3.5]. 


\section{§3.2. Smoothing Mellin corner operators}

Definition 3.2.1. Let $\boldsymbol{g}:=\left(\boldsymbol{g}_{X} ; \boldsymbol{g}_{\mathbb{W}}\right), \boldsymbol{g}_{X}:=(\gamma, \gamma-\mu, \Theta), \boldsymbol{g}_{\mathbb{W}}:=$ $(\delta, \sigma, \Xi)$ and $\boldsymbol{v}=\left(E, F ; J_{-}, J_{+}\right)$, and let $\mathcal{C}_{M+G}(\mathbb{M}, \boldsymbol{g} ; \boldsymbol{v})$ denote the space of all operators $M+G$ for arbitrary $G \in \mathcal{C}_{G}(\mathbb{M}, \boldsymbol{g} ; \boldsymbol{v})$ and

$$
M=t^{\sigma-\delta} \omega(t) \sum_{j=0}^{l} t^{j} \operatorname{op}_{M}^{\alpha_{j}-\frac{m}{2}}\left(f_{j}\right) \widetilde{\omega}(t)
$$

where $\omega, \widetilde{\omega}$ are arbitrary cut-off functions (supported in $[0,1)$ ) and

$$
f_{j} \in \mathcal{M}_{R_{j}}^{-\infty}\left(\mathbb{W}, g_{X}\right) \quad \text { for } R_{j}=\left(R_{j, 1}, R_{j, 2}\right)
$$

where $R_{j, 1} \in \mathbf{A s}_{M+G}\left(X, \boldsymbol{g}_{X} ; \boldsymbol{v}\right)$ and $R_{j, 2} \in \mathbf{A} \mathbf{s}^{\bullet}(\mathbb{W} ; \boldsymbol{v})_{R_{j, 1}}, \delta \geq \alpha_{j}>\delta-j$, and

$$
\pi_{\mathbb{C}} R_{j, 2} \cap \Gamma_{\frac{m+1}{2}-\alpha_{j}}=\emptyset, \quad j=0, \ldots, l .
$$

Here, $\Theta=(-(k+1), 0], k \in \mathbb{N} \cup\{\infty\}$, and $\Xi=(-(l+1), 0], l \in \mathbb{N}$. For infinite $\Xi$ we define $\mathcal{C}_{M+G}(\mathbb{M}, \boldsymbol{g} ; \boldsymbol{v})$ to be the intersection over corresponding operator spaces for finite $\Xi_{l}:=(-(l+1), 0], l \in \mathbb{N}$.

Remark 3.2.2. The specific choice of the cut-off functions $\omega, \widetilde{\omega}$ or of the weights $\alpha_{j}$ affects an operator (3.2.1) only by an element of $\mathcal{C}_{G}(\mathbb{M}, \boldsymbol{g} ; \boldsymbol{v})$. Setting

$$
\sigma_{\mathrm{c}}^{\delta-\sigma-j}(A)(w):=f_{j}(w), \quad 0 \leq j \leq l,
$$

$A \in \mathcal{C}_{M+G}(\mathbb{M}, \boldsymbol{g} ; \boldsymbol{v})$, we have well-defined maps

$$
\sigma_{\mathrm{c}}^{\delta-\sigma-j}: \mathcal{C}_{M+G}(\mathbb{M}, \boldsymbol{g} ; \boldsymbol{v}) \rightarrow \mathcal{M}_{R_{j}}^{-\infty}(\mathbb{W},(\gamma, \gamma-\mu, \Theta)),
$$

and $\sigma_{\mathrm{c}}^{\delta-\sigma-j}(M+G)=0,0 \leq j \leq l$, if and only if $A \in \mathcal{C}_{G}(\mathbb{M}, \boldsymbol{g} ; \boldsymbol{v})$.

Below, for abbreviation we also write

$$
\sigma_{\mathrm{c}}(A):=\sigma_{\mathrm{c}}^{\delta-\sigma}(A)
$$

when $\delta$ and $\sigma$ are known by the context.

Theorem 3.2.3. Let $A \in \mathcal{C}_{M+G}(\mathbb{M}, \boldsymbol{g} ; \boldsymbol{v}), B \in \mathcal{C}_{M+G}(\mathbb{M}, \boldsymbol{h} ; \boldsymbol{w})$ and assume that weight data and bundles in the range of $B$ fit to those in the domain of $A$. We then have $A B \in \mathcal{C}_{M+G}(\mathbb{M}, \boldsymbol{g} \circ \boldsymbol{h} ; \boldsymbol{v} \circ \boldsymbol{w}$ ) (with an evident meaning of $\circ$ in the latter expression). 
The proof of Theorem 3.2.3 is formally similar to a corresponding result in [26, Secton 1.3.1]. In particular, the behaviour of conormal symbols under composition is analogous to the Mellin translation product, known from the calculus for conical singularities with smooth base manifolds.

Theorem 3.2.4. Every $A \in \mathcal{C}_{M+G}(\mathbb{M}, \boldsymbol{g} ; \boldsymbol{v})$ (with the notation for $\boldsymbol{g}$ and $\boldsymbol{v}$ as in Definition 3.2.1) induces continuous operators

$$
A: \mathcal{H}^{s,(\gamma, \delta)}(\mathbb{M} ; \boldsymbol{m}) \rightarrow \mathcal{H}^{\infty,(\gamma-\mu, \sigma)}(\mathbb{M} ; \boldsymbol{n})
$$

and

$$
A: \mathcal{H}_{P}^{s,(\gamma, \delta)}(\mathbb{M} ; \boldsymbol{m}) \rightarrow \mathcal{H}_{Q}^{\infty,(\gamma-\mu, \sigma)}(\mathbb{M} ; \boldsymbol{n})
$$

$\boldsymbol{m}=\left(E, J_{-}\right), \boldsymbol{n}=\left(F, J_{+}\right)$, for all $s \in \mathbb{R}$ and each $P \in \operatorname{As}(X, \mathbb{W}, Z ;(\gamma, \Theta)$, $\left.(\delta, \Xi)^{\bullet},(\delta-(n+1) / 2, \Xi)^{\bullet}\right)$, with some resulting $Q \in \operatorname{As}(X, \mathbb{W}, Z ;(\gamma-\mu, \Theta)$, $\left.(\sigma, \Xi)^{\bullet},(\sigma-(n+1) / 2, \Xi)^{\bullet}\right)$.

The proof of the first assertion of Theorem 3.2.4 is a consequence of the continuity of Mellin pseudo-differential operators in weighted Sobolev spaces that can be shown in the present context anlogously to a corresponding result when the cone base is smooth. The second assertion employs the fact that discrete asymptotics of distributions in $t \in \mathbb{R}_{+}$are translated by the Mellin transform into meromorphy in $w$ and that the Mellin symbols themselves are meromorphic. The inverse Mellin transform then produces resulting asymptotics in $t$ for $t \rightarrow 0$. Concerning $r$-asymptotics the latter conclusion refers to operator-valued symbols that are smoothing and Green in $r$-direction. This implies the asserted mapping property between spaces with asymptotics.

Remark 3.2.5. Let $A \in \mathcal{C}_{M+G}(\mathbb{M}, \boldsymbol{g} ; \boldsymbol{v})$ and assume $\sigma_{M}^{\delta-\sigma}(A)=0$. Then $A$ is compact as an operator $\mathcal{H}^{s,(\gamma, \delta)}(\mathbb{M} ; \boldsymbol{m}) \rightarrow \mathcal{H}^{\widetilde{s},(\gamma-\mu, \sigma)}(\mathbb{M} ; \boldsymbol{n})$ for every $s, \widetilde{s} \in$ $\mathbb{R}$.

\section{§3.3. The edge corner algebra}

Let us fix weight data

$$
\boldsymbol{g}=(\gamma, \gamma-\mu, \Theta ; \delta, \sigma, \Xi)=:\left(\boldsymbol{g}_{X} ; \boldsymbol{g}_{\mathbb{W}}\right)
$$

where $\gamma, \mu, \delta, \sigma \in \mathbb{R}, \Theta=(-(k+1), 0]$ for $k \in \mathbb{N} \cup\{\infty\}, \Xi=(-(l+1), 0]$, $l \in \mathbb{N} \cup\{\infty\}$, and set

$$
\boldsymbol{v}=\left(E, F ; J_{-}, J_{+}\right)
$$


with vector bundles $E, F$ on $\mathbb{M}$ and $J_{-}, J_{+}$on $\mathbb{B}$.

Let $\omega(t)$ and $\widetilde{\omega}(t)$ be cut-off functions supported in $[0,1)$ where $\omega \widetilde{\omega}=\omega$, set $\chi:=1-\omega$, and let $\tilde{\chi} \in C^{\infty}\left(\overline{\mathbb{R}}_{+}\right)$be another function vanishing near zero where $\chi \tilde{\chi}=\chi$.

Definition 3.3.1. The space $\mathcal{C}^{\mu}(\mathbb{M}, \boldsymbol{g} ; \boldsymbol{v})$ is defined to be the set of all operators of the form

$$
A=\omega t^{\delta-\sigma} \operatorname{op}_{M}^{\delta-\frac{m}{2}}(h) \widetilde{\omega}+\chi L \widetilde{\chi}+M+G
$$

for arbitrary $M+G \in \mathcal{C}_{M+G}(\mathbb{M}, \boldsymbol{g} ; \boldsymbol{v}), L \in \mathcal{Y}^{\mu}\left(\mathbb{M}_{\text {reg }}, \boldsymbol{g}_{X} ; \boldsymbol{v}\right)$ and $h(t, w) \in$ $C^{\infty}\left(\overline{\mathbb{R}}_{+}, \mathcal{M}_{R, \mathcal{O}}^{\mu}\left(\mathbb{W}, \boldsymbol{g}_{X} ; \boldsymbol{v}\right)\right.$ for some $R \in \mathbf{A s}_{M+G}\left(X, \boldsymbol{g}_{X} ; \boldsymbol{v}\right)$.

Remark 3.3.2. There is a canonical embedding $\mathcal{C}^{\mu}(\mathbb{M}, \boldsymbol{g} ; \boldsymbol{v}) \hookrightarrow \mathcal{Y}^{\mu}\left(\mathbb{M}_{\mathrm{reg}}\right.$, $\left.\boldsymbol{g}_{X} ; \boldsymbol{v}\right)$, and we have

$$
\mathcal{C}_{M+G}(\mathbb{M}, \boldsymbol{g} ; \boldsymbol{v})=\mathcal{C}^{\mu}(\mathbb{M}, \boldsymbol{g} ; \boldsymbol{v}) \cap \mathcal{Y}^{-\infty}\left(\mathbb{M}_{\mathrm{reg}}, \boldsymbol{g}_{X} ; \boldsymbol{v}\right)
$$

Remark 3.3.3. By definition the elements of $\mathcal{C}^{\mu}(\mathbb{M}, \boldsymbol{g} ; \boldsymbol{v})$ are $2 \times 2$-block matrix operators $A=\left(A_{i j}\right)_{i, j=1,2}$. The space of lower right corners $A_{22}$ consists of the cone algebra $\mathcal{C}^{\mu}\left(\mathbb{B},(\delta-(n+1) / 2, \sigma-(n+1) / 2, \Xi) ;\left(J_{-}, J_{+}\right)\right)$with discrete asymptotics, cf. [26] (recall that $B$ is the edge of $M$ that is a manifold with conical singularities; $\mathbb{B}$ is the associated stretched manifold).

Let us give a more explicit description of interior symbols of operators in $\mathcal{C}^{\mu}(\mathbb{M}, \boldsymbol{g} ; \boldsymbol{v})$ in local coordinates (3.1.1), (3.1.2), (3.1.3). Since our operators outside $\mathbb{M}_{\text {sing }}$ belong to the edge algebra on $\mathbb{M}_{\text {reg }}$, we concentrate on a neighbourhood of $\mathbb{M}_{\text {sing }}$, i.e., on the charts (3.1.1) and (3.1.2). The main contribution comes from the upper left corners. For simplicity, we consider the case of trivial bundles $E$ and $F$ of fibre dimension 1 ; for $\boldsymbol{v}=(\mathbb{C}, \mathbb{C} ; 0,0)$ we omit $\boldsymbol{v}$ in the notation.

Proposition 3.3.4. For $h(t, w) \in C^{\infty}\left(\overline{\mathbb{R}}_{+}, \mathcal{M}_{R, \mathcal{O}}^{\mu}\left(\mathbb{W}, g_{X}\right)\right), \quad R \in$ $\operatorname{As}_{M+G}\left(X, \boldsymbol{g}_{X}\right)$, we have:

(i)

$$
\left(\chi_{\text {corner }}\right)_{*} \omega t^{\delta-\sigma} \operatorname{op}_{M}^{\delta-\frac{m}{2}}(h) \widetilde{\omega} \in L_{\text {cl }}^{\mu}\left(\mathbb{R}_{+} \times \mathbb{R}_{+} \times \Sigma \times \Omega\right)
$$

with an amplitude function of the form $t^{\delta-\sigma} r^{-\mu} p_{\text {corner }}(t, r, x, y, \tau, \varrho, \xi, \eta)$, where 


$$
p_{\text {corner }}(t, r, x, y, \tau, \varrho, \xi, \eta)=\left.\widetilde{p}(t, r, x, y, \widetilde{\tau}, \widetilde{\varrho}, \xi, \widetilde{\eta})\right|_{\widetilde{\tau}=r t \tau, \widetilde{\varrho}=r \varrho, \widetilde{\eta}=r \eta}
$$

for a symbol $\widetilde{p}(t, r, x, y, \widetilde{\tau}, \widetilde{\varrho}, \xi, \widetilde{\eta}) \in S_{\mathrm{cl}}^{\mu}\left(\overline{\mathbb{R}}_{+} \times \overline{\mathbb{R}}_{+} \times \Sigma \times \Omega \times \mathbb{R}_{\widetilde{\tau}, \widetilde{\varrho}, \xi, \widetilde{\eta}}^{2+n+q}\right)$.

(ii)

$$
\left(\chi_{\text {cone }}\right)_{*} \omega t^{\delta-\sigma} \operatorname{op}_{M}^{\delta-\frac{m}{2}}(h) \widetilde{\omega} \in L_{\mathrm{cl}}^{\mu}\left(\mathbb{R}_{+} \times \Delta\right),
$$

and (3.3.4) has an amplitude function of the form $t^{\delta-\sigma} p_{\text {cone }}(t, \widetilde{x}, \tau, \widetilde{\xi})$, where

$$
p_{\text {cone }}(t, \widetilde{x}, \tau, \widetilde{\xi})=\left.\widetilde{p}(t, \widetilde{x}, \widetilde{\tau}, \widetilde{\xi})\right|_{\widetilde{\tau}=t \tau}
$$

for a symbol $\tilde{p}(t, \widetilde{x}, \widetilde{\tau}, \widetilde{\xi}) \in S_{\mathrm{cl}}^{\mu}\left(\overline{\mathbb{R}}_{+} \times \Delta \times \mathbb{R}_{\widetilde{\tau}, \widetilde{\xi}}^{2+n+q}\right)$.

The amplitude functions in (3.3.3) and (3.3.5) refer to the Fourier transform in the respective variables. The characterisations (3.3.2) and (3.3.4) mean that we find amplitude functions $p_{\text {corner }}$ and $p_{\text {cone }}$, such that

$$
\left(\chi_{\text {corner }}\right)_{*} A=\mathrm{Op}_{t, r, x, y}\left(p_{\text {corner }}\right) \bmod L^{-\infty}\left(\mathbb{R}_{+} \times \mathbb{R}_{+} \times \Sigma \times \Omega\right)
$$

and

$$
\left(\chi_{\text {cone }}\right)_{*} A=\mathrm{Op}_{t, \widetilde{x}}\left(p_{\text {cone }}\right) \bmod L^{-\infty}\left(\mathbb{R}_{+} \times \Delta\right),
$$

$A:=\omega \operatorname{op}_{M}^{\delta-(m / 2)}(h) \widetilde{\omega}$.

The proof of Proposition 3.3.4 (ii) is close to that of a similar statement from the cone theory. In fact, if we first substitute the diffeomorphism $\lambda$ : $\mathbb{R}_{+} \rightarrow \mathbb{R}, t \rightarrow \tilde{t}:=-\log t$, for $A_{1}=\left(\chi_{\text {cone }}\right)_{*} A$ we get

$$
\chi_{*} A_{1} \in L_{\mathrm{cl}}^{\mu}(\mathbb{R} \times \Delta)
$$

with an amplitude function $h(-\log \tilde{t}, \widetilde{x}, \gamma-(m / 2)+i \tau, \widetilde{\xi}), \widetilde{t} \in \mathbb{R}$. It follows that $\left(\chi_{*}\right)^{-1} \chi_{*} A_{1} \in L_{\mathrm{cl}}^{\mu}\left(\mathbb{R}_{+} \times \Delta\right)$, where the shape of an amplitude function can be evaluated by applying the standard transformation behaviour of pseudodifferential symbols under push-forwards; this gives us a corresponding Fuchs type degeneracy in the covariable $\tau$ and smoothness in the first variable $t=e^{-\widetilde{t}}$ up to zero. For the proof of Proposition 3.3.4 (i) we can iteratively proceed in a similar manner first with the $t$-variable, according to (ii) and then with the model cone variable $r$. The double degeneracy in $\tau$, i.e., the dependence on $r t \tau$ is due to the fact that the operator-valued Mellin symbols are assumed to be edge-degenerate, i.e, $\tau$ is already multiplied by a factor $r$. 
Let us now consider systems of symbols

$$
\left\{t^{\delta-\sigma} r^{-\mu} p_{i, \text { corner }}(t, r, x, y, \tau, \varrho, \xi, \eta)\right\}_{i=1, \ldots, c}
$$

and

$$
\left\{t^{\delta-\sigma} p_{j, \text { cone }}(t, \widetilde{x}, \tau, \widetilde{\xi})\right\}_{j=1, \ldots, d}
$$

in local coordinates belonging to charts on $\mathbb{M}$ near $\mathbb{M}_{\text {sing }}$

$$
\chi_{i, \text { corner }}: \mathbb{V}_{i} \rightarrow \overline{\mathbb{R}}_{+} \times \overline{\mathbb{R}}_{+} \times \Sigma \times \Omega
$$

and

$$
\chi_{j, \text { cone }}: \mathbb{K}_{j} \rightarrow \overline{\mathbb{R}}_{+} \times \Delta,
$$

respectively, where $\bigcup_{i=1}^{c} \mathbb{V}_{i} \bigcup_{j=1}^{d} \mathbb{K}_{j}$ are assumed to form a neighbourhood of $\mathbb{M}_{\text {sing }}$ in $\mathbb{M}$. Assume for simplicity that all symbols vanish for $t>\varepsilon / 2$. Let us call the system

$$
p:=\left\{p_{i, \text { corner }} ; p_{j, \text { cone }}\right\}_{i=1, \ldots, c, j=1, \ldots, d}
$$

a complete interior symbol on $\mathbb{M}$ near $\mathbb{M}_{\text {sing }}$, if the symbols $p_{i, \text { corner }}$ and $p_{j \text {,cone }}$ behave invariant under symbol push-forward belonging to arbitrary transition maps from (3.3.8) and (3.3.9), modulo symbols of order $-\infty$.

Clearly, the elements in (3.3.6) and (3.3.7) are assumed to be smooth up to $t=0, r=0$ and $t=0$, respectively. (We can easily extend the definition of a complete interior symbol to a full atlas on $\mathbb{M}$ consisting of charts (3.1.1), (3.1.2), (3.1.3) and (3.1.4); far from $\mathbb{M}_{\text {sing }}$ we have the same as in the edge calculus which is left to the reader).

If $h(t, w) \in \mathcal{M}_{R, \mathcal{O}}^{\mu}\left(\mathbb{W}, \boldsymbol{g}_{X}\right)$ is an element vanishing for $t>\varepsilon / 2$, the symbols of $\operatorname{op}_{M}^{\delta-(m / 2)}(h)$ via Proposition 3.3.4 form a complete symbol on $\mathbb{M}$ near $\mathbb{M}_{\text {sing }}$.

$p$ is said to be of order $-\infty$ if all components are of order $-\infty$.

Theorem 3.3.5. $\quad$ To each complete interior corner symbol (3.3.10) on $\mathbb{M}$ near $\mathbb{M}_{\text {sing }}$ (vanishing for $t>\varepsilon / 2$ ) for arbitrary $\gamma, \delta \in \mathbb{R}$ there exists an $h(t, w) \in \mathcal{M}_{R, \mathcal{O}}^{\mu}\left(\mathbb{W}, \boldsymbol{g}_{X}\right)$ for some $R \in \mathbf{A s}\left(X, \boldsymbol{g}_{X}\right)$, where $\boldsymbol{g}_{X}:=(\gamma, \gamma-\mu, \Theta)$, such that the symbols of

$$
\left\{\left(\chi_{i, \text { corner }}\right)_{*} t^{\delta-\sigma} \operatorname{op}_{M}^{\delta-\frac{m}{2}}(h),\left(\chi_{j, \text { cone }}\right)_{*} t^{\delta-\sigma} \operatorname{op}_{M}^{\delta-\frac{m}{2}}(h)\right\}_{i=1, \ldots, c, j=1, \ldots, d}
$$

coincide with $p$, modulo an element of order $-\infty$. 
A proof of Theorem 3.3.5 will be published elsewhere. The result will not be used here, though it illustrates the nature of corner operator spaces in the sense of Definition 3.3.1. Theorem 3.3.5 shows that $\mathcal{C}^{\mu}(\mathbb{M}, \boldsymbol{g} ; \boldsymbol{v})$ is a sufficiently rich operator space, where arbitrary corner-degenerate local symbols are admitted.

The correspondence $p \rightarrow \mathrm{op}_{M}^{\delta-(m / 2)}(h)$ may be regarded as a Mellin operator convention for corner-degenerate symbols and prescribed weights.

Theorem 3.3.6. Every $A \in \mathcal{C}^{\mu}(\mathbb{M}, \boldsymbol{g} ; \boldsymbol{v})$ induces continuous operators

$$
A: \mathcal{H}^{s,(\gamma, \delta)}(\mathbb{M} ; \boldsymbol{m}) \rightarrow \mathcal{H}^{s-\mu,(\gamma-\mu, \sigma)}(\mathbb{M} ; \boldsymbol{n})
$$

and

$$
A: \mathcal{H}_{P}^{s,(\gamma, \delta)}(\mathbb{M} ; \boldsymbol{m}) \rightarrow \mathcal{H}_{Q}^{s-\mu,(\gamma-\mu, \sigma)}(\mathbb{M} ; \boldsymbol{n})
$$

for all $s \in \mathbb{R}$ and every $P \in \operatorname{As}\left(X, \mathbb{W}, Z ;(\gamma, \Theta),(\delta, \Xi)^{\bullet},(\delta-(n+1) / 2, \Xi)^{\bullet}\right)$, with some resulting $Q \in \operatorname{As}\left(X, \mathbb{W}, Z ;(\gamma-\mu, \Theta),(\sigma, \Xi)^{\bullet},(\sigma-(n+1) / 2, \Xi)^{\bullet}\right)$.

The arguments of the proof are similar to those for Theorem 3.2.4. Here, we have to apply them also for the holomorphic Mellin symbols. In addition, for the region far from the corner points we apply Theorem 1.4.3.

Let us now define the principal symbol structure of operators $A \in \mathcal{C}^{\mu}(\mathbb{M}, \boldsymbol{g}$; $\boldsymbol{v})$. First, by Remark 3.3.2 we inherit the principal symbols $\left(\sigma_{\psi}(A), \sigma_{\wedge}(A)\right)$ from $\mathcal{Y}^{\mu}\left(\mathbb{M}_{\mathrm{reg}}, \boldsymbol{g}_{X} ; \boldsymbol{v}\right)$, cf. formulas (1.4.18)-(1.4.21) for the case without parameters. Locally, in the splitting of variables $(t, r, x, y)$ near $\mathbb{M}_{\text {sing }}$ (cf. the notation in the beginning of Section 3.1) we can write

$$
\sigma_{\psi}(A)(t, r, x, y, \tau, \varrho, \xi, \eta), \quad \sigma_{\wedge}(A)(t, y, \tau, \eta) .
$$

Similarly to (1.4.19) we now have associated symbols

$$
\sigma_{\psi, \mathbf{f}}(A)(t, r, x, y, \tau, \varrho, \xi, \eta):=t^{\delta-\sigma} r^{\mu} \sigma_{\psi}(A)\left(t, r, x, y, t^{-1} r^{-1} \tau, r^{-1} \varrho, \xi, r^{-1} \eta\right)
$$

(cf. also the degeneracy in (0.0.4)) and

$$
\sigma_{\wedge, \mathbf{f}}(A)(t, y, \tau, \eta):=t^{\delta-\sigma} \sigma_{\wedge}(A)\left(t, y, t^{-1} \tau, \eta\right)
$$

that are smooth up to $t=0$. We call $\sigma_{\psi, \mathbf{f}}(A)$ and $\sigma_{\wedge, \mathbf{f}}(A)$ the Fuchs type symbols derived from $\sigma_{\psi}(A)$ and $\sigma_{\wedge}(A)$; this is compatible with the terminology of Section 1.4 near the edge far from the corner point.

These symbols have an invariant meaning as bundle homomorphisms

$$
\sigma_{\psi, \mathbf{f}}(A):=\sigma_{\psi, \mathbf{f}}\left(A_{11}\right): \pi_{\mathbb{M}, \mathbf{f}}^{*} E \rightarrow \pi_{\mathbb{M}, \mathbf{f}}^{*} F
$$


for $\pi_{\mathbb{M}, \mathbf{f}}: T_{\mathbf{f}}^{*} \mathbb{M} \backslash 0 \rightarrow \mathbb{M}$, and

$$
\sigma_{\wedge, \mathbf{f}}(A): \pi_{\mathbb{B}, \mathbf{f}}^{*}\left(\begin{array}{c}
\mathcal{K}^{s, \gamma}\left(X^{\wedge}\right) \otimes E^{\prime} \\
\oplus \\
J_{-}
\end{array}\right) \rightarrow \pi_{\mathbb{B}, \mathbf{f}}^{*}\left(\begin{array}{c}
\mathcal{K}^{s-\mu, \gamma-\mu}\left(X^{\wedge}\right) \otimes F^{\prime} \\
\oplus \\
J_{+}
\end{array}\right)
$$

for $\pi_{\mathbb{B}, \mathbf{f}}: T_{\mathbf{f}}^{*} \mathbb{B} \backslash 0 \rightarrow \mathbb{B}$ ( $E^{\prime}$ and $F^{\prime}$ are bundles on the model cone $X^{\wedge}$ obtained by first restricting the original bundles $E$ and $F$ from $\mathbb{W}$ to $X$ and then lifting them to $X^{\wedge}$ by the projection $\left.X^{\wedge} \rightarrow X\right)$. Moreover, $T_{\mathbf{f}}^{*} \mathbb{M}$ and $T_{\mathbf{f}}^{*} \mathbb{B}$ are "compressed" variants of the cotangent bundles $T_{\mathbf{f}}^{*} \mathbb{M}_{\text {int }}$ and $T^{*} \mathbb{B}$, respectively (covectors in $T_{\mathbf{f}}^{*} \mathbb{M}$ near $t=0$ are represented by $(\widetilde{\tau}, \widetilde{\varrho}, \xi, \widetilde{\eta})$, where $(\tau, \varrho, \xi, \eta)=\left(t^{-1} r^{-1} \tau, r^{-1} \varrho, \xi, r^{-1} \eta\right)$ are the "usual" covariables, outside a neighbourhood of $t=0$ this is compatible with the corresponding notion in Section 1.4 ; similarly, covectors in $T_{\mathbf{f}}^{*} \mathbb{B}$ near $t=0$ are represented by $(\widetilde{\tau}, \eta)$ when $(\tau, \eta)=\left(t^{-1} \tau, \eta\right)$ are the usual covariables $)$.

Moreover, we set

$$
\sigma_{\mathrm{c}}(A)(w)=h(0, w)+\sigma_{M}(M+G)(w),
$$

cf. notation (3.2.4). This is a meromorphic operator function

$$
\sigma_{\mathrm{c}}(A)(w): \underset{H^{s-\frac{n+1}{2}}\left(Y, J_{-}\right)}{\mathcal{W}^{s, \gamma}(\mathbb{W}, E)} \rightarrow \underset{H^{s-\mu-\frac{n+1}{2}}\left(Y, J_{+}\right)}{\mathcal{W}^{s-\mu, \gamma-\mu}(\mathbb{W}, F)}
$$

in $w \in \mathbb{C} ; s \in \mathbb{R}$ is arbitrary. Notation of bundles $E, F$ and $J_{-}, J_{+}$are used here in the sense of restrictions of the bundles $E, F$ on $\mathbb{M}$ to $\mathbb{W}$, the base of the corner (recall that $\mathbb{W}$ is a stretched manifold with edge $Y$ ) and of $J_{-}, J_{+}$ to $Y$.

Remark 3.3.7. $A \in \mathcal{C}^{\mu}(\mathbb{M}, \boldsymbol{g} ; \boldsymbol{v})$ implies

$$
\sigma_{\mathrm{c}}(A) \in \mathcal{M}_{S, T}^{\mu}(\mathbb{W}, \boldsymbol{g} ; \boldsymbol{v})
$$

for certain $S \in \mathbf{A s}_{M+G}(X, \boldsymbol{g} ; \boldsymbol{v}), T \in \mathbf{A} \mathbf{s}^{\bullet}(\mathbb{W} ; \boldsymbol{v})_{S}$.

By the principal symbol of $A \in \mathcal{C}^{\mu}(\mathbb{M}, \boldsymbol{g} ; \boldsymbol{v})$ of order $\mu$ we understand the triple

$$
\sigma(A):=\left(\sigma_{\psi, \mathbf{f}}(A), \sigma_{\wedge, \mathbf{f}}(A), \sigma_{\mathrm{c}}(A)\right) .
$$

Remark 3.3.8. $A \in \mathcal{C}^{\mu}(\mathbb{M}, \boldsymbol{g} ; \boldsymbol{v})$ and $\sigma(A)=0$ implies that the operator (3.3.12) is compact for every $s \in \mathbb{R}$. 
In fact, $\sigma(A)=0$ implies the continuity of

$$
A: \mathcal{H}^{s,(\gamma, \delta)}(\mathbb{M} ; \boldsymbol{m}) \rightarrow \mathcal{H}^{s-\mu+1,(\gamma-\mu+\varepsilon, \sigma+\varepsilon)}(\mathbb{M} ; \boldsymbol{n})
$$

for some $\varepsilon>0$. By virtue of Proposition 3.1.2 the space on the right hand side is compactly embedded into $\mathcal{H}^{s-\mu,(\gamma-\mu, \sigma)}(\mathbb{M} ; \boldsymbol{n})$.

In the following theorem we set

$$
\begin{array}{ll}
\boldsymbol{g}=\left(\boldsymbol{g}_{X}, \boldsymbol{g}_{\mathbb{W}}\right) & \text { for } \boldsymbol{g}_{X}:=(\gamma-\nu, \gamma-(\nu+\mu), \Theta), \boldsymbol{g}_{\mathbb{W}}:=(\beta, \sigma, \Xi), \\
\boldsymbol{h}=\left(\boldsymbol{h}_{X}, \boldsymbol{h}_{\mathbb{W}}\right) & \text { for } \boldsymbol{h}_{X}:=(\gamma-\nu, \gamma-\nu, \Theta), \boldsymbol{h}_{\mathbb{W}}:=(\delta, \beta, \Xi),
\end{array}
$$

and $\boldsymbol{v}:=\left(E_{0}, F ; J_{0}, J_{+}\right), \boldsymbol{w}:=\left(E, E_{0} ; J_{-}, J_{0}\right)$.

Theorem 3.3.9. $A \in \mathcal{C}^{\mu}(\mathbb{M}, \boldsymbol{g} ; \boldsymbol{v}), B \in \mathcal{C}^{\nu}(\mathbb{M}, \boldsymbol{h} ; \boldsymbol{w})$ implies $A B \in$ $\mathcal{C}^{\mu+\nu}(\mathbb{M}, \boldsymbol{g} \circ \boldsymbol{h} ; \boldsymbol{v} \circ \boldsymbol{w})$ where

$$
\sigma(A B)=\sigma(A) \sigma(B)
$$

(with componentwise composition of the first two symbols, while $\sigma_{\mathrm{c}}(A B)(w)=$ $\left.\left(T^{-\delta+\beta} \sigma_{\mathrm{c}}(A)\right) \sigma_{\mathrm{c}}(B),\left(T^{\beta} f\right)(w)=f(w+\beta)\right)$. If $A$ or $B$ belongs to the subspace with subscript $M+G(G)$ then the same is true of the composition.

Proof. We have assumed (for simplicity) that $\mathbb{M}$ is compact. Therefore, the composition $A B$ exists in the sense of continuous operators in respective weighted spaces, cf. the first assertion of Theorem 3.3.6. Moreover, by construction we have $A \in \mathcal{Y}^{\mu}\left(\mathbb{M}_{\text {reg }}, \boldsymbol{g}_{X} ; \boldsymbol{v}\right)$ a similar relation for $B$, cf. Remark 3.3.2. Then Theorem 1.4.7 gives us $A B \in \mathcal{Y}^{\mu+\nu}\left(\mathbb{M}_{\mathrm{reg}},(\boldsymbol{g} \circ \boldsymbol{h})_{X} ; \boldsymbol{v} \circ \boldsymbol{w}\right)$, together with the symbol rules from the edge calculus. Furthermore, the operators $A$ and $B$ near $\mathbb{M}_{\text {sing }}$ also behave like edge operators, where the Fourier transform along the $\mathbb{R}_{+}$component of the edge is replaced by the Mellin transform in $t \in \mathbb{R}_{+}$. The edge calculus with the Mellin transform in one of the local edge coordinates is analogous to that with the Fourier transform; then we have again a composition result in the corresponding Mellin-Fourier edge calculus, including the corresponding symbol rule. This gives us the multiplicativity of the $\sigma_{\psi, \mathbf{f}}$ and $\sigma_{\wedge, \mathbf{f}}-$ components. It remains to observe that operators in our cone-edge calculus near $\mathbb{M}_{\text {sing }}$ formally behave like cone operators with operator-valued symbols, similarly to the cone algebra with smooth base, cf. [26, Chapter 2] or [29, Chapter 2]. In the present situation the base is a compact (stretched) manifold $\mathbb{W}$ with edges, but compositions can analogously be evaluated. In particular, we get compositions of the same nature, and, in particular, the asserted symbol rule for conormal symbols. Finally, the last statement when in 
a composition a factor belongs to a subclass with subscript $M+G$ is a consequence of Remark 3.3.2. If one of the factors is a Green operator, the assertion follows from the definition of Green operators together with Theorem 3.3.6 and a similar construction for formal adjoints (that can easily be identified as elements in a modification of our calculus with shifted order and weight indices, cf. formula (3.3.1).

\section{§3.4. Ellipticity and regularity with asymptotics}

We now turn to the ellipticity in the edge-corner algebra on $\mathbb{M}$ with trace and potential conditions on $\mathbb{B}$. Notation for weight data and bundles are used as in Definition 3.3.1.

Definition 3.4.1. An operator $A \in \mathcal{C}^{\mu}(\mathbb{M}, \boldsymbol{g} ; \boldsymbol{v})$ is said to be elliptic if

(i) both (3.3.15) and (3.3.16) are isomorphisms,

(ii) (3.3.18) is an isomorphism for all $w \in \Gamma_{(m+1) / 2-\delta}$, for some $s \in \mathbb{R}$ where $m=1+n+q$.

Recall that, according to the meaning of the notation in (3.3.15), (3.3.16), the isomorphisms are required to hold up to $r=0$ and $t=0$ in corresponding stretched coordinates. In particular, ellipticity of $A \in \mathcal{C}^{\mu}(\mathbb{M}, \boldsymbol{g} ; \boldsymbol{v})$ implies ellipticity in the sense of $\mathcal{Y}^{\mu}\left(\mathbb{M}_{\text {reg }}, \boldsymbol{g}_{X} ; \boldsymbol{v}\right)$, cf. Remark 3.3.2.

Remark 3.4.2. If (3.3.18) is an isomorphism for an $s=s_{0} \in \mathbb{R}$ then so is for all $s \in \mathbb{R}$.

In fact, $\left.\sigma_{c}(A)\right|_{\Gamma_{(m+1) / 2-\delta}} \in \mathcal{Y}^{\mu}\left(\mathbb{W}, \boldsymbol{g}_{X} ; \boldsymbol{v} ; \Gamma_{(m+1) / 2-\delta}\right)$ is parameter-dependent elliptic. Then (3.3.18) is a family of Fredholm operators for every $s$, but kernel and cokernel are independent of $s$.

In the following theorem we set

$$
\boldsymbol{g}^{-1}:=(\gamma-\mu, \gamma, \Theta ; \sigma, \delta, \Xi), \quad \boldsymbol{v}^{-1}:=\left(F, E ; J_{+}, J_{-}\right)
$$

and $\boldsymbol{g}_{l}:=(\gamma, \gamma, \Theta ; \sigma, \sigma, \Xi), \boldsymbol{v}_{l}:=\left(E, E ; J_{-}, J_{-}\right), \boldsymbol{g}_{r}=(\gamma-\mu, \gamma-\mu, \Theta ; \delta, \delta ; \Xi)$, $\boldsymbol{v}_{r}:=\left(F, F ; J_{+}, J_{+}\right)$.

For simplicity we now assume $\mathbb{M}$ to be compact.

Theorem 3.4.3. Let $A \in \mathcal{C}^{\mu}(\mathbb{M}, \boldsymbol{g} ; \boldsymbol{v})$ be elliptic. Then there is a parametrix $P \in \mathcal{C}^{-\mu}\left(\mathbb{M}, \boldsymbol{g}^{-1} ; \boldsymbol{v}^{-1}\right)$ in the sense

$$
1-P A \in \mathcal{C}_{G}\left(\mathbb{M}, \boldsymbol{g}_{l} ; \boldsymbol{v}_{l}\right), \quad 1-A P \in \mathcal{C}_{G}\left(\mathbb{M}, \boldsymbol{g}_{r} ; \boldsymbol{v}_{r}\right) .
$$


Proof. First of all, $A_{\text {int }}:=\left.A\right|_{\mathbb{M}_{\mathrm{reg}}} \in \mathcal{Y}^{\mu}\left(\mathbb{M}_{\mathrm{reg}}, \boldsymbol{g}_{X} ; \boldsymbol{v}\right)$ is elliptic in the sense of Definition 1.4 .8 and has a parametrix $P_{\text {int }} \in \mathcal{Y}^{-\mu}\left(\mathbb{M}_{\text {reg }}, \boldsymbol{g}_{X}^{-1} ; \boldsymbol{v}^{-1}\right)$ by Theorem 1.4.9. Restricting $A_{\text {int }}$ to a neighbourhood $(0, \varepsilon) \times \mathbb{W}$, where $[0, \varepsilon) \times \mathbb{W}$ denotes a neighbourhood of $\mathbb{M}_{\text {sing }}$ in $\mathbb{M}$, we now observe that $A_{\varepsilon}:=$ $t^{\delta-\sigma} \mathrm{op}_{M}^{\delta-(m / 2)}(h) \in \mathcal{Y}^{\mu}\left((0, \varepsilon) \times \mathbb{W}, \boldsymbol{g}_{X} ; \boldsymbol{v}\right)$ is also elliptic for a sufficiently small $\varepsilon>0$, and that there is an $f(t, w) \in C^{\infty}\left(\overline{\mathbb{R}}_{+}, \mathcal{M}_{S, \mathcal{O}}^{-\mu}\left(\mathbb{W}, \boldsymbol{g}_{X}^{-1} ; \boldsymbol{v}^{-1}\right)\right.$ for a certain $S$, such that $P_{\varepsilon}:=t^{\sigma-\delta} \operatorname{op}_{M}^{\sigma-(m / 2)}(f)$ is a parametrix of $A_{\varepsilon}$ in $(0, \varepsilon) \times \mathbb{W}$. In the sequel we simply set $\varepsilon=\infty$ which can be reached by a simple diffeomorphism $(0, \varepsilon) \rightarrow \mathbb{R}_{+}$. To construct $f$ we first consider the case $\sigma=\delta$. We then have the Mellin-Leibniz product

$$
f(t, w) \#_{M} h(t, w) \sim \sum_{k=0}^{\infty} \frac{1}{k !}\left(\partial_{w}^{k} f\right)(t, w)\left(-t \partial_{t}\right)^{k} h(t, w),
$$

cf. [26, Section 1.2.4, Theorem 18], or [15] for a similar situation. Here, it can be carried out as an asymptotic sum in $C^{\infty}\left(\overline{\mathbb{R}}_{+}, \mathcal{Y}^{0}\left(\mathbb{W},(\gamma, \gamma, \Theta) ; \boldsymbol{v}_{l} ; \Gamma_{(m+1) / 2-\delta}\right)_{\widetilde{S}}\right.$ for some asymptotic type $\widetilde{S}$. This allows us to invert $\left.h(t, w)\right|_{\overline{\mathbb{R}}_{+} \times \Gamma_{(m+1) / 2-\delta}}$ with respect to $\#_{M}$. Denote by $f_{1}(t, w) \in C^{\infty}\left(\overline{\mathbb{R}}_{+}, \mathcal{Y}^{-\mu}\left(\mathbb{W}, \boldsymbol{g}_{X}^{-1} ; \boldsymbol{v}^{-1} ; \Gamma_{(m+1) / 2-\delta}\right) S_{1}\right.$ the resulting inverse, where $S_{1}$ is certain asymptotic type. According to Remark 2.1.6 from $f_{1}(t, w)$ we can pass to an $f(t, w) \in C^{\infty}\left(\overline{\mathbb{R}}_{+}, \mathcal{M}_{S_{1}, \mathcal{O}}^{-\mu}\left(\mathbb{W}, \boldsymbol{g}_{X}^{-1} ; \boldsymbol{v}^{-1}\right)\right)$ such that $f(t, w) \#_{M} h(t, w)-1 \in C^{\infty}\left(\overline{\mathbb{R}}_{+}, \mathcal{M}_{S_{2}, \mathcal{O}}^{-\infty}\left(\mathbb{W}, \boldsymbol{g}_{X}^{-1} ; \boldsymbol{v}^{-1}\right)\right)$ for a certain $S_{2}$. We then obtain, in particular,

$$
f(0, w) h(0, w)=1+l(w)
$$

for some $l \in \mathcal{M}_{S_{2}, \mathcal{O}}^{-\infty}\left(\mathbb{W}, \boldsymbol{g}_{X, l} ; \boldsymbol{v}_{l}\right)$. Next we apply the ellipticity condition (ii) in Definition 3.4.1. It tells us that $h(0, w)$ defines an isomorphism (3.3.18) on the weight line $\Gamma_{(m+1) / 2-\delta}$. Theorem 2.2.4 yields $h^{-1}(0, w) \in \mathcal{M}_{R, V}^{-\mu}\left(\mathbb{W}, \boldsymbol{g}_{X}^{-1} ; \boldsymbol{v}^{-1}\right)$ for certain $R, V$.

Thus, using (3.4.2) it follows that $\left(f(0, w)-h^{-1}(0, w)\right) h(0, w)=: l(w)$, i.e., $f(0, w)-h^{-1}(0, w)=l(w) h^{-1}(0, w) \in \mathcal{M}_{\widetilde{R}, \widetilde{V}}^{-\infty}\left(\mathbb{W}, g_{X}^{-1} ; \boldsymbol{v}^{-1}\right)$, cf. Theorem 2.2.3. Thus, we find a smoothing Mellin operator $M_{1}:=-\omega \operatorname{op}_{M}^{\delta-(m / 2)}\left(\left.l h^{-1}\right|_{t=0}\right) \widetilde{\omega}$ such that

$$
\sigma_{\mathrm{c}}\left(\omega \mathrm{op}_{M}^{\delta-\frac{m}{2}}(f) \widetilde{\omega}+M_{1}\right)=\sigma_{\mathrm{c}}^{-1}(A)
$$

We now form the operator $P_{1}:=\omega \operatorname{op}_{M}^{\delta-(m / 2)}(f) \widetilde{\omega}+\chi P_{\text {int }} \widetilde{\chi}+M_{1}$. Then

$$
1-P_{1} A \in \mathcal{C}_{M+G}\left(\mathbb{M}, \boldsymbol{g}_{l} ; \boldsymbol{v}_{l}\right), \quad 1-A P_{1} \in \mathcal{C}_{M+G}\left(\mathbb{M}, \boldsymbol{g}_{r} ; \boldsymbol{v}_{r}\right),
$$

cf. Remark 3.3.2, and $\sigma\left(P_{1}\right)=\sigma^{-1}(A)$ as a consequence of (3.4.3). In particular, the leading conormal symbols in $1-P_{1} A$ and $1-A P_{1}$ vanish. This allows 
us to apply a formal Neumann series argument to improve $P_{1}$ to the desired $P$, by setting

$$
P:=\left\{\sum_{j=0}^{N}(-1)^{j}\left(1-P_{1} A\right)^{j}\right\} P_{1} .
$$

$N$ may be taken to be finite when the weight interval $\Xi$ is finite; for infinite $\Xi$ we can carry out (3.4.4) in the sense of an asymptotic sum, cf. also [26, Section 2.2.2] for a similar (but simpler) situation with infinite sums of smoothing Mellin operators. It remains to look at the case of arbitrary $\sigma, \delta$. The only change in this case is a corresponding shift of weights with respect to the corner axis variable, both for the original operator and for the parametrix that may be commuted through operators, up to a shift of Mellin symbols in $w$.

Corollary 3.4.4. For the parametrix $P$ in Theorem 3.4 .3 we have

$$
\sigma(P)=\left(\sigma_{\psi, \mathbf{f}}^{-1}(A), \sigma_{\wedge, \mathbf{f}}^{-1}(A), T^{\delta-\sigma} \sigma_{\mathrm{c}}^{-1}(A)(w)\right),
$$

$\left(T^{\beta} f\right)(w)=f(w+\beta)$.

In fact, it suffices to apply Theorem 3.3.9.

Corollary 3.4.5. If $A \in \mathcal{C}^{\mu}(\mathbb{M}, \boldsymbol{g} ; \boldsymbol{v})$ is elliptic, the operator (3.3.12) is Fredholm for every $s \in \mathbb{R}$.

This is a consequence of Theorem 3.4.3, Corollary 3.4.4, and Remark 3.3.8.

Theorem 3.4.6. Let $A \in \mathcal{C}^{\mu}(\mathbb{M}, \boldsymbol{g} ; \boldsymbol{v})$ be elliptic, and $u \in \mathcal{H}^{-\infty,(\gamma, \delta)}(\mathbb{M}$; $\boldsymbol{m})$. Then we have

$$
A u=f \in \mathcal{H}^{s-\mu,(\gamma-\mu, \sigma)}(\mathbb{M} ; \boldsymbol{n}) \Rightarrow u \in \mathcal{H}^{s,(\gamma, \delta)}(\mathbb{M} ; \boldsymbol{m})
$$

and

$$
A u=f \in \mathcal{H}_{Q}^{s-\mu,(\gamma-\mu, \sigma)}(\mathbb{M} ; \boldsymbol{n}) \Rightarrow u \in \mathcal{H}_{P}^{s,(\gamma, \delta)}(\mathbb{M} ; \boldsymbol{m})
$$

for each $s \in \mathbb{R}$ and every $Q \in \operatorname{As}\left(X, \mathbb{W}, Z ;(\gamma-\mu, \Theta),(\sigma, \Xi)^{\bullet},(\sigma-(n+1) / 2, \Xi)^{\bullet}\right)$ with some resulting $P \in \operatorname{As}\left(X, \mathbb{W}, Z ;(\gamma, \Theta),(\delta, \Xi)^{\bullet},(\delta-(n+1) / 2, \Xi)^{\bullet}\right)$.

Proof. Set $C:=1-P A$, where $P$ is a parametrix of $A$, cf. relation (3.4.1). Then $A u=f$ gives us $P A u=(1-C) u=P f$, i.e., $u=P f+C u$. Applying Theorem 3.3.6 to $P$ and Definition 3.1.4 to $C$ we immediately obtain the assertion. 
Remark 3.4.7. Let $A \in \mathcal{C}^{\mu}(\mathbb{M}, \boldsymbol{g} ; \boldsymbol{v})$ be elliptic, and let $A_{s}$ denote the operator (3.3.12) in the space $\mathcal{H}^{s,(\gamma, \delta)}(\mathbb{M} ; \boldsymbol{m})$ for some $s \in \mathbb{R}$. Then there is a finite-dimensional subspace $\mathcal{N}_{+} \subset \mathcal{H}_{P}^{\infty,(\gamma, \delta)}(\mathbb{M} ; \boldsymbol{m})$ for some

$$
P \in \operatorname{As}\left(X, \mathbb{W}, Z ;(\gamma, \Theta),(\delta, \Xi)^{\bullet},\left(\delta-\frac{n+1}{2}, \Xi\right)^{\bullet}\right)
$$

such that $\mathcal{N}_{+}=\operatorname{ker} A_{s}$. Moreover, there is a finite-dimensional subspace $\mathcal{N}_{-} \subset$ $\mathcal{H}_{R}^{\infty,(\gamma-\mu, \sigma)}(\mathbb{M} ; \boldsymbol{n})$ for some

$$
R \in \operatorname{As}\left(X, \mathbb{W}, Z ;(\gamma-\mu, \Theta),(\sigma, \Xi)^{\bullet},\left(\sigma-\frac{n+1}{2}, \Xi\right)^{\bullet}\right)
$$

such that $\mathcal{N}_{-} \cap \operatorname{im} A_{s}=\{0\}$ and $\mathcal{N}_{-}+\operatorname{im} A_{s}=\mathcal{H}^{s-\mu,(\gamma-\mu, \sigma)}(\mathbb{M} ; \boldsymbol{n})$. The space $\mathcal{N}_{+}$is independent of $s, \mathcal{N}_{-}$can be chosen to be independent of $s$. In addition, the parametrix of $A$ can be chosen in such a way that the remainders in relation (3.4.1) are projections, where $1-P A$ projects to $\operatorname{ker} A$ and $1-A P$ to a complement of $\operatorname{im} A_{s}$ for every $s \in \mathbb{R}$.

This is an easy consequence of Theorem 3.4.6. The arguments are analogous to those from the standard pseudo-differential calculus on a smooth compact manifold.

Remark 3.4.8. Assume $A \in \mathcal{C}^{\mu}(\mathbb{M}, \boldsymbol{g} ; \boldsymbol{v})$ satisfies the condition (i) (not necessarily condition (ii)) of Definition 3.4.1, then there is a $P \in \mathcal{C}^{-\mu}(\mathbb{M}$, $\left.\boldsymbol{g}^{-1} ; \boldsymbol{v}^{-1}\right)$ such that

$$
1-P A \in \mathcal{C}_{M+G}\left(\mathbb{M}, \boldsymbol{g}_{l} ; \boldsymbol{v}_{l}\right), \quad 1-A P \in \mathcal{C}_{M+G}\left(\mathbb{M}, \boldsymbol{g}_{r} ; \boldsymbol{v}_{r}\right) .
$$

In fact, the first steps in the proof of Theorem 3.4.3 give us an element $P_{0} \in \mathcal{C}^{-\mu}\left(\mathbb{M}, \boldsymbol{g}^{-1} ; \boldsymbol{v}^{-1}\right)$, where $1-P_{0} A$ and $1-A P_{0}$ restrict to elements in the respective $\mathcal{Y}^{-\infty}$-classes on $\mathbb{M}_{\text {reg }}$. Then it suffices to apply Theorem 3.3 .9 and relation (3.3.1).

\section{$\S 3.5$. Examples and remarks}

Let us return to corner-degenerate differential operators of the form (0.0.3), (0.0.4), and give an explicit description of the principal symbol levels. For simplicity, we assume $\mathbb{M}=\overline{\mathbb{R}}_{+} \times \mathbb{W}$, where the stretched corner base $\mathbb{W}$ is a compact $C^{\infty}$ manifold with boundary $\partial \mathbb{W} \cong X \times Y$. The corner conormal symbol of the operator (0.0.3) is the operator family

$$
\sigma_{\mathrm{c}}(A)(w):=\sum_{k=0}^{\mu} b_{k}(0) w^{k}: \mathcal{W}^{s, \gamma}(\mathbb{W}) \rightarrow \mathcal{W}^{s-\mu, \gamma-\mu}(\mathbb{W}),
$$


$w \in \mathbb{C}$. The choice of $\gamma \in \mathbb{R}$ is to be specified in connection with ellipticity. Locally in a neighbourhood of the corner (for $t>0$ ) and of $\partial \mathbb{W}$ the operator (0.0.3) has the form (0.0.4) and is edge-degenerate, where $(t, y) \in \mathbb{R}_{+} \times \Omega$ are local coordinates on the edge. The principal edge symbol has the form

$$
\begin{aligned}
\sigma_{\wedge}(A)(t, y, \tau, \eta)= & r^{-\mu} \sum_{\substack{j+k+|\alpha| \leq \mu\\
}} a_{j k \alpha}(t, 0, y)(-r t i \tau)^{j}\left(-r \frac{\partial}{\partial r}\right)^{k}(r \eta)^{\alpha} \\
& : \mathcal{K}^{s, \gamma}\left(X^{\wedge}\right) \rightarrow \mathcal{K}^{s-\mu, \gamma-\mu}\left(X^{\wedge}\right),
\end{aligned}
$$

where $r$ is the axial variable of the model cone of the local wedge. The interior principal symbol of order $\mu$ is as usual. In particular, if $\sigma_{\psi}\left(a_{j k \alpha}\right)(t, r, y ; x, \xi)$ denotes the homogeneous principal symbol of $a_{j k \alpha}$ of order $\mu-(j+k+|\alpha|)$ in local coordinates $x \in \Sigma$ (corresponding to a chart on $X$ ) with covaribles $\xi$, we have

$$
\begin{aligned}
& \sigma_{\psi}(A)(t, r, x, y, \tau, \varrho, \xi, \eta) \\
& \quad=t^{-\mu} r^{-\mu} \sum_{j+k+|\alpha|=\mu} \sigma_{\psi}\left(a_{j k \alpha}\right)(t, r, y ; x, \xi)(-r t i \tau)^{j}(-r i \varrho)^{k}(r \eta)^{\alpha}
\end{aligned}
$$

Moreover, if we look at the representation $(0.0 .3)$ and denote by $(\widetilde{x}, \widetilde{\xi})$ points on $T^{*}\left(\mathbb{W}_{\text {reg }}\right) \backslash 0$, we have

$$
\sigma_{\psi}(A)(t, \widetilde{x}, \tau, \widetilde{\xi})=t^{-\mu} \sum_{k=0}^{\mu} \sigma_{\psi}\left(b_{k}\right)(t ; \widetilde{x}, \widetilde{\xi})(-i t \tau)^{k},
$$

where $\sigma_{\psi}\left(b_{k}\right)(t ; \widetilde{x}, \widetilde{\xi})$ is the homogeneous principal symbol of $b_{k}$ as a differential operator on $\mathbb{W}_{\text {reg }}=\mathbb{W} \backslash \partial \mathbb{W}$ of order $\mu-k$.

The considerations of the previous sections on $\sigma_{\psi, \mathbf{f}}$ and $\sigma_{\wedge, \mathbf{f}}-$ versions of $\sigma_{\psi}$ and $\sigma_{\wedge}$ remain in force, though we now prefer to employ the standard variables and covariables in the chosen splittings of coordinates. Let us write for a moment $\sigma_{\psi, \text { corner }}(A)$ and $\sigma_{\psi, \text { cone }}(A)$ for the expressions (3.5.1) and (3.5.2), respectively.

Definition 3.5.1. An operator $A$ of the form (0.0.3) is said to be $\sigma_{\psi^{-}}$ elliptic if

(i) $\sigma_{\psi}(A) \neq 0$ on $T^{*}\left(\mathbb{R}_{+} \times \mathbb{W}_{\text {reg }}\right) \backslash 0$,

(ii) $t^{\mu} r^{\mu} \sigma_{\psi, \text { corner }}(A)\left(t, r, x, y, r^{-1} t^{-1} \tau, r^{-1} \varrho, \xi, r^{-1} \eta\right) \neq 0$ for $(\tau, \varrho, \xi, \eta) \neq 0$ and all $(t, r, x, y)$, up to $t=0, r=0$, 
(iii) $t^{\mu} \sigma_{\psi, \text { cone }}(A)\left(t, \widetilde{x}, t^{-1} \tau, \widetilde{\xi}\right) \neq 0$ for all $(\tau, \widetilde{\xi}) \neq 0$ and all $(t, \widetilde{x})$ up to $t=0$.

Observe that Laplace-Beltrami operators for corner-degenerate metrics as mentioned in the introduction are $\sigma_{\psi}$-elliptic.

Proposition 3.5.2. Let $A$ be $\sigma_{\psi}$-elliptic. Then for every $(t, y) \in \overline{\mathbb{R}}_{+} \times$ $Y$ there exists a countable set $D(t, y) \subset \mathbb{C}$, where $D(t, y) \cap S_{\left[c, c^{\prime}\right]}$ is finite for every $c \leq c^{\prime}$, such that

$$
t^{\mu} \sigma_{\wedge}(A)\left(t, y, t^{-1} \tau, \eta\right): \mathcal{K}^{s, \gamma}\left(X^{\wedge}\right) \rightarrow \mathcal{K}^{s-\mu, \gamma-\mu}\left(X^{\wedge}\right)
$$

is a Fredholm operator for $(\tau, \eta) \neq 0$ for every $\gamma \notin\{\operatorname{Re} z: z \in D(t, y)\}$ and all $s \in \mathbb{R}$.

Clearly, for $(\tau, \eta) \neq 0$ we then know that

$$
\sigma_{\wedge}(A)(t, y, \tau, \eta): \mathcal{K}^{s, \gamma}\left(X^{\wedge}\right) \rightarrow \mathcal{K}^{s-\mu, \gamma-\mu}\left(X^{\wedge}\right)
$$

is a Fredholm operator for the admitted $\gamma$ and all $s \in \mathbb{R}$.

Let us now assume that $A$ is $\sigma_{\psi}$-elliptic and that there is a choice of $\gamma \in \mathbb{R}$ such that the condition $\gamma \notin\{\operatorname{Re} z: z \in D(t, y)\}$ is fulfilled for all $t \in \overline{\mathbb{R}}_{+}$and all $y \in Y$ (clearly, there are many examples where this is the case; instead of $t \in \overline{\mathbb{R}}_{+}$, for reasonable examples it suffices to require the condition for $0 \leq t<\varepsilon$ for some $\varepsilon>0$ ). Then we can try to complete the Fredholm family (3.5.3) to a family of isomorphisms

$$
\left(\begin{array}{c}
\sigma_{\wedge, \mathbf{f}}(A) \sigma_{\wedge, \mathbf{f}}(K) \\
\sigma_{\wedge, \mathbf{f}}(T) \sigma_{\wedge, \mathbf{f}}(Q)
\end{array}\right)(t, y, \widetilde{\tau}, \eta): \pi_{\mathbb{B}, \mathbf{f}}^{*} \mathcal{K}^{s, \gamma}\left(X^{\wedge}\right) \rightarrow \pi_{\mathbb{B}, \mathbf{f}}^{*} \mathcal{K}^{s-\mu, \gamma-\mu}\left(X^{\wedge}\right)
$$

cf. formula (3.3.14), here for $\delta-\sigma=-\mu$, and $\mathbb{B}=\overline{\mathbb{R}}_{+} \times Y$ in the notation of (3.3.16). The existence of corresponding vector bundles $J_{-}, J_{+} \in \operatorname{Vect}(\mathbb{B})$ and of additional entries $\sigma_{\wedge, \mathbf{f}}(T)$, etc. is not always guaranteed. There is a topological obstruction, similarly to that of Atiyah and Bott [1] for the existence of Shapiro-Lopatinskij elliptic boundary conditions in the case of boundary value problems, see also Boutet de Monvel [3] and Rempel and Schulze [17]. We now assume that this obstruction vanishes for $A$; again there are many examples where this is fulfilled, for instance, for Laplace-Beltrami operators to corner-degenerate metrics (in the opposite case a scenario similarly to [31] applies; it will be published in a joint paper with Seiler [33]).

The nature of the extra entries in (3.5.5) corresponds to that in the general calculus before. They are simlpy homogeneous principal components of Green 
symbols in the sense of an anlogue of Definition 1.1.1 for $U=\overline{\mathbb{R}}_{+} \times \Omega$ (in local coordinates belonging to charts on $\left.Y, \Omega \subseteq \mathbb{R}^{q}\right)$, now with covariables $(\widetilde{\tau}, \eta)$, where $j_{ \pm}$are the fibre dimensions of $J_{ \pm}$. We may (and, for simplicity, will) choose homogeneous principal symbols of elements $g(t, y, \widetilde{\tau}, \eta) \in \mathcal{R}_{G}^{\mu}\left(\overline{\mathbb{R}}_{+} \times \Omega \times\right.$ $\left.\mathbb{R}^{1+q}, \boldsymbol{g} ; \boldsymbol{w}\right)_{\mathcal{O}}$ for $\boldsymbol{w}=\left(1,1 ; j_{-}, j_{+}\right)$, cf. formula (1.1.17), where $\boldsymbol{g}=(\gamma, \gamma-$ $\mu,(-\infty, 0])$. Recall that homogeneity refers to the group actions (1.1.5). In the present case we have $g=\left(g_{i j}\right)_{i, j=1,2}$, where $g_{11}$ vanishes because we are talking about additional trace and potential etc. entries.

From the local Green symbols $g$ (with respect to a covering of $Y$ by charts $\chi: U \rightarrow \Omega)$ we can pass to a parameter-dependent family of Green operators $f(t, \widetilde{\tau})$ on $\mathbb{W}$ by forming a sum over expressions $\left(\chi^{-1}\right)_{*} \mathrm{Op}_{y}(\omega \varphi g \widetilde{\varphi} \widetilde{\omega})(t, \widetilde{\tau})$, with cut-off functions $\omega(r), \widetilde{\omega}(r)$ supported in a small neighbourhood of $r=0$, $\varphi \in C_{0}^{\infty}(\Omega)$, where $\chi^{*} \varphi$ belongs to a partition of unity on $Y$ (subordinate to the covering of $Y$ ) and $\widetilde{\varphi} \in C_{0}^{\infty}(\Omega)$ where $\varphi \widetilde{\varphi}=\widetilde{\varphi}$.

We thus obtain a family

$$
f(t, \widetilde{\tau}) \in C^{\infty}\left(\overline{\mathbb{R}}_{+}, \mathcal{Y}_{G}^{\mu}(\mathbb{W}, \boldsymbol{g} ; \boldsymbol{v})_{\mathcal{O}}\right)
$$

for $\boldsymbol{v}=\left(1,1 ; J_{-}, J_{+}\right)$.

Using (0.0.3), we form the operator family $h_{11}(t, w)=\sum_{k=0}^{\mu} b_{k}(t) w^{k}$, and then

$$
\left(\begin{array}{cc}
\left.h_{11}(t, w)\right|_{w=-i \widetilde{\tau}} & f_{12}(t, \widetilde{\tau}) \\
f_{21}(t, \widetilde{\tau}) & f_{22}(t, \widetilde{\tau})
\end{array}\right)
$$

is parameter-dependent elliptic in $\mathcal{Y}^{\mu}\left(\mathbb{W}, \boldsymbol{g} ; \boldsymbol{v} ; \mathbb{R}_{\widetilde{\tau}}\right)$ for every $t \in \overline{\mathbb{R}}_{+}$. Interpreting the variable $\widetilde{\tau}$ in the entries $f_{i j}$ as $-\operatorname{Im} w$ for $w \in \Gamma_{0}$ we can apply Theorem 2.1.5 to $\left(\left.f_{i j}(t, \widetilde{\tau})\right|_{\tilde{\tau}=-\operatorname{Im} w}\right)_{i, j=1,2, i+j>2}$ for $\beta=0$ and $l=0$ in the $t$-dependent variant, cf. Remark 2.1.6. This gives us a corresponding element $\left(h_{i j}(t, w)\right)_{i, j=1,2, i+j>2}$ in $C^{\infty}\left(\overline{\mathbb{R}}_{+}, \mathcal{M}_{\mathcal{O}, \mathcal{O}}^{\mu}(\mathbb{W}, \boldsymbol{g} ; \boldsymbol{v})\right)$. Together with the upper left corner $h_{11}(t, w)$ that is given by the differential operator $(0.0 .3)$ we now obtain an element $h(t, w)=\left(h_{i j}(t, w)\right)_{i, j=1,2} \in C^{\infty}\left(\overline{\mathbb{R}}_{+}, \mathcal{M}_{\mathcal{O}, \mathcal{O}}^{\mu}(\mathbb{W}, \boldsymbol{g} ; \boldsymbol{v})\right)$ where $h(t, \beta+i \tau) \in C^{\infty}\left(\overline{\mathbb{R}}_{+}, \mathcal{Y}^{\mu}\left(\mathbb{W}, \boldsymbol{g} ; \boldsymbol{v} ; \Gamma_{\beta}\right)\right)$ is parameter-dependent elliptic for every $\beta \in \mathbb{R}$ (uniformly in compact $\beta$-intervals).

Setting $h(w):=h(0, w)$, we are now in the situation of Remark 2.2.1. It follows that there is a countable set $D \subset \mathbb{C}$ such that the operators (2.2.2) are isomorphisms for all $w \in \mathbb{C} \backslash D$. This determines weights $\delta \in \mathbb{R}$ where the operator

$$
\mathcal{A}:=\left(\begin{array}{cc}
A & 0 \\
0 & 0
\end{array}\right)+t^{-\mu} \mathrm{op}_{M}^{\delta-\frac{m}{2}}\left(\left(h_{i j}\right)_{i, j=1,2, i+j>2}\right)
$$


(with $A$ being given by (0.0.3)) belongs to the corner algebra $\mathcal{C}^{\mu}(\mathbb{M}, \boldsymbol{k} ; \boldsymbol{v})$ for $\boldsymbol{k}:=((\gamma, \gamma-\mu,(-\infty, 0]) ;(\delta, \delta-\mu,(-\infty, 0])), \mathbb{M}=\overline{\mathbb{R}}_{+} \times \mathbb{W}$, and has an elliptic conormal corner symbol $\sigma_{\mathrm{c}}(\mathcal{A})(w)=h(w)$ on the weight line $\Gamma_{(m+1) / 2-\delta}$. In other words, $\mathcal{A}$ is elliptic in the sense of Definition 3.4.1 for $\sigma=\delta-\mu$.

As noted in Section 3.4 the compactness of $\mathbb{M}$ is not an essential assumption, except for Corollary 3.4.5. The other results of Section 3.4 have obvious analogues for general $\mathbb{M}$, and they can be applied to our elliptic operator $\mathcal{A}$. To get the Fredholm property we can easily modify our example by completing $\mathbb{M}=\overline{\mathbb{R}}_{+} \times \mathbb{W}$ for $t \rightarrow \infty$ by another corner point to make the new configuration compact with two corners, cf. the author's joint paper [7] with Fedosov and Tarkhanov for a similar situation, where the base is a manifold with conical singularities.

Concerning regularity of solutions with asymptotics in our iterated sense, it is not easy to explicity evaluate the asymptotic types. However, in concrete examples with, for instance, extra symmetry properties of the metric and of the coefficients, this should be an elementary fact, though it may require separate papers.

Let us finally have a look at a Cartesian product $M:=B_{1} \times B_{2}$ of two manifolds $B_{1}, B_{2}$ with conical singularities. We then have $\mathbb{M}=\mathbb{B}_{1} \times \mathbb{B}_{2}$. Assume for simplicity that $B_{1}$ and $B_{2}$ have one conical singularity with base manifolds $X_{1}$ and $X_{2}$, respectively. Then $M$ is locally near $M^{\prime \prime}=\{v\}$ (the corner point) modelled by $X_{1}^{\Delta} \times X_{2}^{\Delta}$, or, in stretched form, by $\left(\overline{\mathbb{R}}_{+} \times X_{1}\right) \times$ $\left(\overline{\mathbb{R}}_{+} \times X_{2}\right)$. Let $\left(r^{i}, x^{i}\right)$ denote the variables on $X_{i}^{\wedge}, i=1,2$. Then, for our operator calculus we have two non-equivalent choices of corner and cone axis variables, namely $r^{1}=: t, r^{2}=: r$ or $r^{2}=: t$ and $r^{1}=: r$. In the first case $M$ is interpreted near $v$ as a cone with base $X_{1} \times B_{2}$ and in the second case as a cone with base $B_{1} \times X_{2}$. This example illustrates the role of the system of singular charts on $\mathbb{M}$, where different choices give rise to edge-corner algebras that are different with respect to the corner conormal symbol structure.

\section{References}

[1] Atiyah, M. F. and Bott, R., The index problem for manifolds with boundary, Coll. Differential Analysis, Tata Institute Bombay, Oxford University Press, 1964, pp. 175186.

[2] Behm, S., Pseudo-differential operators with parameters on manifolds with edges, $\mathrm{Ph}$. D. thesis, University of Potsdam, 1995.

[3] Boutet de Monvel, L., Boundary problems for pseudo-differential operators, Acta Math., 126 (1971), 11-51.

[4] Dorschfeldt, Ch., Algebras of pseudo-differential operators near edge and corner singularities, Math. Res., 102, Akademie Verlag, Berlin, 1998. 
[5] Egorov, Ju. V. and Schulze, B.-W., Pseudo-differential operators, singularities, applications, Operator Theory, Advances and Applications, 93, Birkhäuser Verlag, Basel, 1997.

[6] Fedosov, B. V., Schulze, B.-W. and Tarkhanov, N. N., On the index formula for singular surfaces, Pacific J. Math., 191 (1999), 25-48.

[7] — Analytic index formulas for elliptic corner operators, Ann. Inst. Fourier, 52 (2002), to appear.

[8] Gil, J. B., Full asymptotic expansion of the heat trace for non-self-adjoint elliptic cone operators, Preprint math. 0004161, Temple University, Dept. Math., Philadelphia, April 2000.

[9] Gil, J. B., Schulze, B.-W and Seiler, J., Cone pseudo-differential operators in the edge symbolic calculus, Osaka J. Math., 37 (2000), 219-258.

[10] Gohberg, I. C. and Sigal, E. I. An operator generalization of the logarithmic residue theorem and the theorem of Rouché, Math. USSR Sbornik, 13 (1971), 603-625.

[11] Kapanadze, D. and Schulze, B.-W., Crack theory and edge singularities, Preprints 2001/05-2001/09, Institute for Mathematics, Potsdam, 2001.

[12] Kondrat'ev, V. A., Boundary value problems for elliptic equations in domains with conical points, Trudy Mosk. Mat. Obshch., 16 (1967), 209-292.

[13] Krainer, T. and Schulze, B.-W., Long-time asymptotics for parabolic operators in the pseudodifferential cone algebra approach, Preprint 99/12, Institute for Mathematics, Potsdam, 1999.

[14] - Long-time asymptotics with geometric singularities in the spatial variables, Preprint 2000/17, Institute for Mathematics, Potsdam, 2000.

[15] Lewis, J. E. and Parenti, C., Pseudo-differential operators of Mellin type, Comm. Partial Differential Equations 8 (1983), 447-544.

[16] Maniccia, L. and Schulze, B.-W., An algebra of meromorphic corner symbols, Preprint, Institute for Mathematics, Potsdam, 2001, in preparation.

[17] Rempel, S. and Schulze, B.-W., Index theory of elliptic boundary problems, AkademieVerlag, Berlin, 1982.

[18] — Complete Mellin and Green symbolic calculus in spaces with conormal asymptotics, Ann. Global Anal. Geom., 4 (1986), 137-224.

[19] Rozenblum, G., On some analytical index formulas related to operator-valued symbols, Preprint 2000/16, Institute for Mathematics, Potsdam, 2000.

[20] Schrohe, E. and Schulze, B.-W., Boundary value problems in Boutet de Monvel's calculus for manifolds with conical singularities I, Adv. Partial Differ. Equ. (Pseudo-Differential Calculus and Mathematical Physics), Akademie Verlag, Berlin, 1994, pp. 97-209.

[21] — Boundary value problems in Boutet de Monvel's calculus for manifolds with conical singularities II, Adv. Partial Differ. Equ. (Boundary Value Problems, Schrödinger Operators, Deformation Quantization), Akademie Verlag, Berlin, 1995, pp. 70-205.

[22] Schulze, B.-W., Ellipticity and continuous conormal asymptotics on manifolds with conical singularities, Math. Nachr., 136 (1988), 7-57.

[23] — Corner Mellin operators and reduction of orders with parameters, Ann. Scuola Norm. Sup. Pisa Cl. Sci., 16 (1989), 1-81.

[24] — - Pseudo-differential operators on manifolds with edges, Symposium Partial Differential Equations, Holzhau 1988, Teubner-Texte zur Mathematik, 112, Teubner, Leipzig, 1989, pp. 259-287.

[25] — Mellin representations of pseudo-differential operators on manifolds with corners, Ann. Glob. Anal. Geom., 8 (1990), 261-297.

[26] — Pseudo-differential operators on manifolds with singularities, North-Holland, Amsterdam, 1991.

[27] — - The Mellin pseudo-differential calculus on manifolds with corners, Symposium: Analysis in Domains and on Manifolds with Singularities, Breitenbrunn 1990, TeubnerTexte zur Mathematik, 131, Teubner, Leipzig, 1992, pp. 208-289.

[28] — - Pseudo-differential boundary value problems, conical singularities, and asymp- 
totics, Akademie Verlag, Berlin, 1994.

[29] Schulze, B.-W., Boundary value problems and singular pseudo-differential operators, J. Wiley, Chichester, 1998.

[30] - Iterated asymptotics on corner manifolds, Proceedings PDE Conference Wuhan, April 5-9, 1999, World Scientific Publishing Co. of Singapore, 2000, pp. 283-296.

[31] — An algebra of boundary value problems not requiring Shapiro-Lopatinskij conditions, J. Funct. Anal., 179 (2001), 374-408.

[32] — - Operator algebras with symbol hierarchies on manifolds with singularities, Adv. Partial Differ. Equ. (Approaches to Singular Analysis) J. Gil, D. Grieser and Lesch M. (eds.), Oper. Theory Adv. Appl., Birkhäuser Verlag, Basel, 2001, pp. 167-207.

[33] Schulze, B.-W. and Seiler, J., A Toeplitz analogue of the edge algebra, Preprint, Institute for Mathematics, Potsdam, 2002, to appear.

[34] Schulze, B.-W., Sternin, B. Ju. and Shatalov, V., On the index of differential operators on manifolds with conical singularities, Ann. Global Anal. Geom., 16 (1998), 141-172.

[35] Schulze, B.-W. and Tarkhanov, N. N., Ellipticity and parametrices on manifolds with cuspidal edges, Geometric Aspects in Partial Differential Equations, B. Booss-Bavnbeck and K. Wojciechowski (eds.), Contemp. Math., 242, Amer. Math. Society, Providence, R.I., 1999, pp. 217-256.

[36] Sobolev, S. L., Einige Anwendungen der Funktionalanalysis auf Gleichungen der mathematischen Physik, Akademie-Verlag, Berlin, 1964.

[37] Sternin, B. Ju., Elliptic and parabolic equations on manifolds with boundary consisting of components of different dimensions, Trudy. Moskov Mat. Obshch., 15 (1966), 346-382. 